\title{
On a mathematical model for case hardening of steel
}

\author{
vorgelegt von \\ Dott.ssa \\ Lucia Panizzi
}

Von der Fakultät II , Mathematik und Naturwissenschaften der Technischen Universität Berlin zur Erlangung des akademischen Grades Doktor der Naturwissenschaften

Dr. rer. nat.

sowie

der Classe di Scienze der Scuola Normale Superiore di Pisa

als

Diploma di Perfezionamento in Matematica per la Tecnologia e l'Industria

genehmigte Dissertation

Promotionsausschuss:

Berichter/Gutachter: Prof. A. Fasano (Univerisità di Firenze)

Berichter/Gutachter: Prof. D. Hömberg (Technische Universität Berlin)

Gutachter: Prof. L. Formaggia (Politecnico di Milano)

Gutachter: Prof. M. Primicerio (Università di Firenze)

Gutachter: Prof. F. Tröltzsch (Technische Universität Berlin)

Gutachter: Prof. P. Wittbold (Technische Universität Berlin)

Tag der wissenschaftlichen Aussprache: 05.03.2010

Berlin 2010 



\section{Eidesstattliche Versicherung}

Hiermit erkläre ich, dass die vorliegende Dissertation:

On a mathematical model for case hardening of steel

selbständig verfasst wurde. Die benutzten Hilfsmittel und Quellen wurden von mir angegeben, weitere wurden nicht verwendet. 


\section{Erklärung}

Hiermit erkläre ich, dass die Anmeldung meiner Promotionsabsicht früher nicht bei einer anderen Hochschule oder einer anderen Fakultät beantragt wurde. Teile meiner Dissertation sind darüber hinaus schon veröffentlich worden, welche im Folgenden aufgelistet sind:

- D. Hömberg, A. Fasano, L. Panizzi A mathematical model for case hardening of steel. angenommen zur Veröffentlichung in "Mathematical Models and Methods in Applied Sciences" (M3AS), 2009.

- P. Krejčí, L. Panizzi.

Regularity and uniqueness in quasilinear parabolic systems.

angenommen zur Veröffentlichung in "Applications of Mathematics", 2009. 


\section{Compendio}

Nonostante la disponibilità di numerosi nuovi materiali, l'acciaio rimane il materiale di base della moderna società industriale. L'uso dell' acciaio con peculiari caratteristiche (durezza, resistenza all'uso, malleabilità etc.) è perciò assai diffuso in molti settori della tecnica. La sua importanza è dovuta soprattutto al fatto che larga parte delle proprietà desiderate a scopi industriali è ottenibile tramite un trattamento termico mirato. Svariati trattamenti termici dell'acciaio sono noti e utilizzati sin dall'antichità. Essi si basano sul riscaldamento e raffreddamento del materiale in diversi cicli e a diverse velocità. La durezza, per esempio, può essere ottenuta tramite riscaldamento seguito da rapido raffreddamento. Un processo che mira all'indurimento di uno strato esterno soltanto, lasciando la parte interna duttile, prende il nome di 'case hardening'. Tuttora, nell'industria, l'approccio più diffuso ai trattamenti termici è basato sull' esperienza accumulata negli anni e sui dati ricavati da tentativi sperimentali, fino ad ottenere un risultato soddisfacente. Questo modo di procedere presenta lo svantaggio di necessitare di un gran numero di esperimenti, risultando quindi dispendioso e non facilmente riproducibile e controllabile. Da ciò nasce l'esigenza di un controllo più preciso del processo, volto a minimizzare il consumo energetico e, di conseguenza, economico. A tal fine, gli apporti della ricerca nel campo dell'ingegneria, della fisica, della chimica e anche - in gran misura - della matematica sono cruciali.

La prima indagine analitica della trasformazione di fase solido-solido che ha luogo durante i trattamenti termici nell' acciaio risale ai lavori pionieristici di M. Avrami e A. Kolmogorov negli anni trenta dello scorso secolo. Da quel momento, essendo chiara l'utilità dell' approccio analitico, le ricerche sulla modellizzazione matematica a riguardo si sono estese e numerosi risultati sperimentali sono stati pubblicati riguardo le trasformazioni di fase nell'acciaio. Ciononostante, la prima trattazione matematica rigorosa di questo problema può essere fatta risalire a A. Visintin con alcuni lavori della metà degli anni ottanta. La quantità degli studi dedicati alla modellizzazione delle trasformazioni di fase è notevolmente aumentata nelle ultime due decadi, a seguito della crescente domanda e dell'ampio impiego nell'industria di processi in cui le trasformazioni di fase rivestono un ruolo di primo piano. Un Istituto dedito da anni ad approfondite ricerche sull'argomento è il Weierstraß Insitute für Angewandte Analysis und Stochastik (WIAS) di Berlino, dove la presente tesi è stata scritta. Esso vanta una decennale ricerca di matematica applicata nell'ambito dell'analisi e dell' ottimizzazione dei più significativi trattamenti termici dell'acciaio, in collaborazione con Istituti di ingegneria e di scienza dei materiali.

Dal punto di vista della modellizzazione matematica, un trattamento termico in generale può essere descritto come combinazione dei processi di trasferimento termico e di trasformazioni di fase, che sono responsabili delle diverse strutture cristalline possibili nell'acciaio. Questi fenomeni fisici sono descrivibili attraverso un sistema di equazioni alle derivate parziali di evoluzione e, nel caso di trattamenti termico-chimici, equazioni aggiuntive sono necessarie per descrivere la diffusione di altre sostanze chimiche. Il processo industriale oggetto della presente tesi è la più diffusa variante di 'case hardening', cosiddetta 'gas carburizing', che prevede la variazione della composizione chimica dell'acciaio, nello strato superficiale, mediante la diffusione di un gas costituito prin- 
cipalmente da carbonio. Infatti, per ottenere un certo grado di durezza, è necessaria una quantità percentuale di carbonio, che non è presente negli acciai di base più usati, i quali sono a basso contenuto di carbonio.

La presente tesi si occupa della modellizzazione matematica, a livello macroscopico, del 'gas carburizing', della sua analisi matematica, e infine presenta un lavoro di simulazione del modello proposto.

La parte introduttiva della tesi è volta alla descrizione dei principali fenomeni fisici che hanno luogo nell'acciaio al variare della temperatura, per giungere alla formulazione di un modello matematico. Il modello è costituito da un sistema di due equazioni paraboliche alle derivate parziali, che descrivono l'evoluzione della temperatura e quella del contenuto in carbonio all'interno della componente meccanica, accoppiate a due equazioni differenziali ordinarie che descrivono le transizioni di fase (solido-solido) che hanno luogo nell'acciaio sottoposto al trattamento termico. Vengono affrontate le questioni di esistenza e unicità di una soluzione del problema al bordo associato al sistema di equazioni del modello completo, con condizioni al bordo di terzo tipo per le equazioni paraboliche. Successivamente si analizza un problema correlato a quello di partenza, vale a dire il sistema quasilineare costituito dalle due equazioni paraboliche alle derivate parziali, riuscendo ad indebolire sensibilmente le ipotesi che sembravano necessarie nella prima parte, col vantaggio di rispecchiare con maggior fedeltà alcune caratteristiche fisiche del problema. In questo caso, la tecnica usata è ispirata ad un metodo proposto da J. Nečas originalmente per la risoluzione di equazioni ellittiche con condizioni al bordo lineari, ma che consente di trattare anche una condizione al bordo non lineare per l'equazione descrivente l'evoluzione della temperatura. Altrettanto utili nella trattazione dell'unicità e continuità della soluzione rispetto ai dati sono alcune classi di spazi di Sobolev anisotropici, introdotti da O. V. Besov, che risultano particolarmente adatti a trattare il problema della diversa regolarità della soluzione in direzione tangenziale e normale. L'analisi è valida nei casi di dimensione spaziale minore o uguale a tre, tenendo conto che quello rilevante nelle applicazioni è il caso tridimensionale. In conclusione vengono presentate alcune simulazioni che mostrano l'applicabilità del modello considerato, finalizzate ad essere comparate con risultati sperimentali. 


\section{Zusammenfassung}

Stahl ist, obwohl die Entwicklung und Nutzung neuer vielseitiger Werkstoffe und Materialen immer bedeutsamer wird, nach wie vor der Grundwerkstoff einer modernen industriellen Gesellschaft. Er ist der am meist verwendete metallische Werkstoff. Mit seinen garantierten Eigenschaften (Festigkeit, Korrosionsverhalten, Verformbarkeit, Schweißeignung usw.) deckt er in der Technik ein weites Anwendungsfeld ab. Nach der klassischen Definition ist Stahl eine Eisen-Kohlenstoff-Legierung, die weniger als 2,06 \% (Masse) Kohlenstoff enthält. Die grundsätzliche Bedeutung des Kohlenstoffs ergibt sich aus seinem Einfluss auf das Phasenumwandlungsverhalten und damit auf die mechanischen Eigenschaften des Stahls, was vor allem man sich in der Wärmebehandlung zu Nutze macht. Die Wärmebehandlung von Stahl blickt auf eine über tausend jährige Geschichte zurück. Die Grundidee bei der Wärmebehandlung besteht darin, einen Stahl mit bestimmten gewünschten Eigenschaften durch eine geeignete Kombination von kontrollierter Erhitzung und Abkühlung zu erzeugen. So kann z.B. die Härte eines Stahls durch starke Erwärmung gefolgt von einer schnellen Abkühlung deutlich erhöht werden. Ein spezielles Verfahren, das sich auf eine relative dünne, oberflächennahe Schicht beschränkt, heißt 'Randschichthärten'. Bei diesem Verfahren wird nur die äußerste Schicht des Werkstücks gehärtet, was den Verschleiß bei mechanischer Beanspruchung deutlich verringert. Das Innere hingegen behält seine Duktilität was insbesondere für die Dauerfestigkeit eines Bauteils von Vorteil ist.

In der vorliegenden Arbeit wird eine spezielle verfahren des Randschichthärten untersucht, dass so genannte 'Einsatzhärten'. Bei diesem Verfahren wird in die Oberfläche von Werkstücken aus kohlenstoffarmen Stahl von außen durch Diffusion Kohlenstoff eingebracht. Das Werkstück bezieht dabei den zur Diffusion notwendigen Kohlenstoff aus einer kohlenstoffreichen Gasatmosphäre. Auch in der heutigen hoch technologisierten Industrie ist es gängige Methode die Prozessparameter für das Einsatzhärten experimentell zu ermitteln. In der Regel werden Erfahrungswerte mit zusätzlichen experimentellen Studien kombiniert um eine optimale Wärmebehandlungstrategie für ein bestimmtes Bauteil zu entwickeln. Ein großer Nachteil hierbei ist, dass trotz aller Optimierungsversuche oft viele kostspielige und zeitaufwändige Experimente notwendig sind. Es bleibt also festzustellen, dass die Eisen- und Stahlwerkstoffe mit ihren vielfältigen Anwendungsmöglichkeiten in der Industrie eine zentrale Rolle einnehmen, wenngleich aus den unterschiedlichen Anforderungen auch die Notwendigkeit erwächst, die Produktionsprozesse in ihrer ganzen Komplexität zu verstehen und zu beherrschen. In diesem Punkt können die Ingenieurwissenschaften, die Physik, die Chemie, aber auch in einem hohem Masse die Mathematik entscheidende Beiträge liefern.

Die mathematische Beschreibung von fest-fest-Phasenumwandlungen in Stahl, die in den dreißiger Jahren des letzten Jahrhunderts mit den ersten Arbeiten von M. Avrami und A. Kolmogorov seinen Anfang nahm, ist bis heute Gegenstand weitreichender Untersuchungen, wie sich eindrucksvoll Anhand der zahlreichen Veröffentlichungen über die Modellierung und die numerische Simulation von diffusiven Phasenumwandlungen in Stahl belegen lässt.

Eine erste mathematische Studie zu Austenit-Perlit-Phasenübergängen, ist von A. Visintin in den achtziger Jahren des letzten Jahrhunderts vorgelegt worden. Aufgrund der 
zunehmenden Bedeutung der Phasenumwandlungen in der industriellen Anwendung ist die Zahl der wissenschaftlichen Publikationen zu dieser Problematik n den letzten Jahrzehnten stark angestiegen. In diesem Zusammenhang ist auch das Weierstraß Institut für angewandte Analysis und Stochastik (WIAS Berlin) zu nennen wo im Rahmen des Forschungsschwerpunkts „Optimale Steuerung von Produktionsprozessen“ der Forschungsgruppe 4 seit vielen Jahren an diesem Thema gearbeitet wird und dessen Erfahrung in großem Maße in die vorliegende Arbeit eingeflossen ist.

Aus Sicht der mathematischen Modellierung lässt sich die Wärmebehandlung von Stahl als eine Kombination von Wärmeleitungs- und Phasenentwicklungsprozessen verstehen, die letztendlich für die Entstehung der verschiedenen Kristallsorten im Stahl verantwortlich sind. Diese Vorgänge werden in der Regel durch ein System von Evolutionsgleichungen beschrieben, das im Wesentlichen aus Gleichungen für die zeitliche Entwicklung der verschiedenen Phasen, der Temperatur und, im Falle des Einsatzhärtens, aus einer Diffusionsgleichung für den Kohlenstoff besteht.

Ziel der vorliegenden Arbeit ist die mathematische Beschreibung des Einsatzhärtens auf makrospkopischer Ebene, die mathematische Untersuchung der Modellgleichungen und numerische Simulationen, die die Eigenschaften des Modells illustrieren.

Im ersten Kapitel werden die grundsätzlichen physikalischen Phänomene beschrieben, die im Stahl bei Variation der Temperatur ablaufen, was schliesslich auf die Modellgleichungen führt, die ein nichlineares, gekoppeltes System von gewönlichen und partiellen Differentialgleichungen darstellen. Die beiden partiellen Differentialgleichungen parabolischen Typs beschreiben die Temperaturentwicklung und die Evolution der Kohlenstoffkonzentration im Bauteil. Die gewöhnlichen Differentialgleichungen modellieren die Phasenentwicklung. Im zweiten Kapitel wird die Frage nach der Existenz einer eindeutigen Lösung des Rand-Anfangswertproblems diskutiert, das aus den Modellgleichungen zusammen mit Randbedingungen dritter Art entsteht. Um die für den Beweis der Eindeutigkeit einer Lösung des Gesamtmodells relativ starken Voraussetzungen abschwächen zu können, wird zusätzlich ein Teilproblem eingeführt und analysiert, das aus einem quasilinearen, parabolischen System von nur zwei gekoppelten, partiellen Differentialgleichungen besteht. Für dieses System konnte eine Technik entwickelt werden, die auf einer Methode von J. Necas basiert. Ursprünglich war diese Methode zur Lösung von elliptischen Differentialgleichungen mit linearen Randbedingungen gedacht, sie konnte aber auf den parabolischen Fall mit einer nichtlinearen Randbedingung für die Temperatur erweitert werden.

Ein weiteres wichtiges Werkzeug zur Behandlung der Frage der Eindeutigkeit der Lösung und deren stetiger Abhängikeit von den Daten sind die anisotropischen SobolevRäume die von O.V. Besov eingeführt wurden. Sie ermöglichen es Probleme mit unterschiedlichen Regularitäten in tangentialer und normaler Richtung zu behandeln. Die bewiesenen Existenz- und Eindeutigkeitssätze gelten für Raumdimensionen kleiner gleich drei, wobei der dreidimensionale Fall aus Anwendungsicht die grösste Relevanz besitzt. Die numerischen Simulationen am Ende der Arbeit, die durch einen Vergleich mit experimentellen Ergebnissen noch validiert werden können, belegen die prinzipielle Anwendbarkeit des entwickelten Modells. 


\section{Acknowledgments}

My research activity has been possible thanks to the financial support of Scuola Normale Superiore di Pisa, thanks to the hospitality of the Weierstrass Institute for Applies Analysis and Stochastic (WIAS) in Berlin, where I spent almost three years, and thanks to the cooperation of the Technische Universität Berlin.

First, I would like to thank both my supervisors Prof. Antonio Fasano (University of Florence) and Prof. Dietmar Hömberg (TUB, WIAS) for their willingness in enabling a thesis project in cooperation and for their continuous support. Moreover, I would like to thank Prof. Dietmar Hömberg for his help in obtaining a prolongation grant at WIAS and for making all the facilities there available to me.

I am particularly indebted to Pavel Krejčí (Institute of Mathematics, Academy of Sciences of the Czech Republic) for his cooperation and patience in conveying to me his deep mathematical knowledge.

I thank all the colleagues and friends I met during my $\mathrm{PhD}$ period in Pisa, who spent with me a joyful year in a very motivating and pleasant atmosphere.

I would like to express my sincere gratitude to Daniela Kern, Christian Meyer, Oliver Rott, Nataliya Togobytska and Wolf Weiss, from WIAS, for their kindness and their constant availability in taking time for discussions. I especially thank Oliver, for the patient help with the german translations, for many precious suggestions and for his valuable support.

Finally, I would like to thank my family, in particular my parents and my sister Silvia for their faith in my abilities and encouragement in helping me to complete this research. 


\section{Contents}

Introduction $\quad x i$

1 Physical background and modelling 1

1.1 Phase transitions in iron-carbon equilibrium diagram . . . . . . . . . 1

1.2 Time-temperature-transformation diagrams . . . . . . . . . . . . 3

1.3 Heat treatment of steel: case hardening . . . . . . . . . . . . . . . 9

1.4 Modelling of gas carburizing . . . . . . . . . . . . . . . . . . 10

1.4.1 Carbon diffusion in austenite . . . . . . . . . . . . . . . . 10

1.4.2 Phase transformations . . . . . . . . . . . . . . . 12

1.4.3 Heat transfer . . . . . . . . . . . . . . . . . . . 13

1.4.4 Complete model of gas carburizing . . . . . . . . . . . . 14

2 Analysis of the complete model $\quad 17$

2.1 Problem statement and strategy . . . . . . . . . . . . . . 17

2.2 Assumptions and main results . . . . . . . . . . . . . . . . . . . . 18

2.3 Proof of existence . . . . . . . . . . . . . . . . . . . . . . . . 19

2.4 Proof of uniqueness . . . . . . . . . . . . . . . . 33

3 Analysis of a related quasilinear parabolic system 39

3.1 Problem statement and strategy . . . . . . . . . . . . . . . 39

3.2 Main results . . . . . . . . . . . . . . . . . . . . . . . 41

3.3 Proof of existence . . . . . . . . . . . . . . . . . . . . . . 43

3.4 Proof of regularity f . . . . . . . . . . . . . . . . . . 45

3.5 An anisotropic embedding theorem . . . . . . . . . . . 56

3.6 Proof of continuous data dependence . . . . . . . . . . . . . . . 62

4 Numerical results $\quad 69$ 


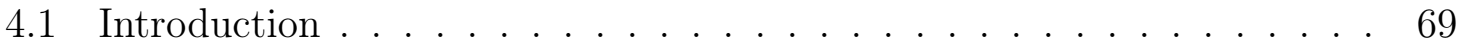

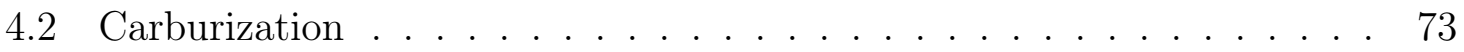

4.3 Quenching . . . . . . . . . . . . . . . . . . 77

4.4 Simulation of the complete process $\ldots \ldots \ldots \ldots$. . . . . . 82

$\begin{array}{llr}5 & \text { Conclusions } & 89\end{array}$

$\begin{array}{ll}\text { Bibliography } & 92\end{array}$ 


\section{Introduction}

\section{Outline}

Despite the creation of numerous new functional materials, steel is still the basic material for the sustainable development of modern industrial society. Its applications are very diverse and widespread in all major branches of industry. Heat treatment of steel is a fundamental process which dates back thousands of years. Thanks to the tremendous flexibility of steel to various kinds of treatments, it is now possible to produce it with a large variety of desired properties.

The basic principle involved in heat treatment is the process of heating and cooling. In steel for example, hardness can be achieved by heating followed by rapid cooling. In general, two important properties of ferrous materials are contact fatigue strength and wear resistance, which depend mainly on the physical and chemical properties of a superficial layer. A special treatment acting on a relatively thin superficial layer of steel workpieces is called case hardening, because its aim is to harden just the workpiece case (i.e an encasing layer), letting the inner part softer. In nowadays high-technology industry the approach to case hardening often involves trial and error methods, based on previous experiences and empirical analysis. This kind of procedure requires costly and time-consuming experiments.

Due to its importance, there is a huge technical literature on the subject of heat treatment of steel, mostly of engineering type. On the other hand, the thermal processing of steel has also attracted the attention of mathematicians. The mathematical description of solid-solid phase transitions in steel started with the seminal works of Avrami [4] and Kolmogorov [30] in the thirties of last century. Since then the subject has been widely studied and, stimulated by the development of ever-faster computer hardware, numerous papers were published on the numerical simulation of the diffusion controlled phase transitions in steel. The first analytical investigation of phase transitions in steel, concerned with austenite-pearlite transition, is reported by A. Visintin [49]. Similar models have been studied in connection with polymerisation models [2]. The amount of works dedicated to the modelling of the phase transitions in steel has been increasing in the last decades, due to the numerous applications in industry. Studies about phase transitions in metallic alloys have been particularly undertaken at WIAS (Weierstrass Institute for Applied Analysis and Stochastics), where this thesis has been written, exploiting the large experience accumulated on the subject.

From the modelling point of view, heat treatments of steel include heat transfer and 
transition processes involving the many different crystalline species in steel. Occasionally, as in the specific case of carburizing processes, more equations are needed to describe the evolution of added chemicals.

The heat treatment that we want to investigate in the present work is the most widely used variant of case hardening, named gas carburizing. Carbon is indeed the key to the hardening of steel by the heating and quick cooling mechanism. If the used steel does not contain sufficient carbon to provide the required hardness, then its composition is altered only on the surface layer so that it can become hard during subsequent cooling. In carburizing, the workpiece is heated up to a certain temperature, then it is brought in contact with an environment of sufficient carbon potential to cause the carbon absorption at the surface and, by diffusion favoured by the high temperature, to create a carbon concentration gradient inside the workpiece. Then the workpiece is rapidly cooled down, so that the carbon diffusivity drops practically to zero and carbon atoms remain frozen within the iron lattice. This causes an atomic disorder and results in distortion of the lattice which manifests itself in the form of hardness and/or strength. Regarding this process, we do not know any reference to previously formulated models, though the literature dedicated to modelling phase transition in steel during heat treatments (like induction or flame hardening) is quite large (see the remarkable group of papers [19], [20], [21], [23]). Within the engineering literature, the process of gas carburizing is divided and investigated in two distinct parts: first carburization, then cooling. In the studies regarding the carburization stage, the focus is almost exclusively on the carbon evolution, described through a diffusion equation often with constant coefficients and disregarding of the coupling with temperature. This approach does not permit, for instance, to take into account further diffusion of carbon into the workpiece in a lower temperature range during the stage preceding quenching, which nevertheless is very important for the control of the carbon profile and the accuracy of the whole process. Among them, we quote for example [9], [12], [16], [44], [45] and [46]. A huge amount of studies regarding exclusively the quenching of steel in correlation with its hardening is available (see for instance [13], [25], [39] and the monograph [48]). To the best of our knowledge a complete model including all the possible effects has not yet been examined.

\section{Objective}

The aim of the present thesis is the development and analysis of a mathematical model for the process of gas carburizing, taking into account the basic characteristics under the actual conditions of the industrial process and relating the quality of the treatment with the technological parameters of the process. In view of the lack of mathematical studies concerning this process, we start with an analytical study, which focuses on the examination of the mathematical model and on the related mathematical questions. Then we present some numerical simulations to illustrate the applicability of our model to a concrete example.

The work is organised as follows. 
Chapter 1 starts with some fundamental concepts regarding steel from the point of view of materials science and with the description of its most important metallurgical properties. We focus on a special type of case hardening process, named gas carburizing and come to the formulation of a mathematical model for this process, at the macroscopic level, in term of a set of differential equations, one for the diffusion of carbon, one for the thermal evolution, both coupled with the equations describing the evolution of the phase fractions, which are, in our case, two ordinary differential equations.

In Chapter 2 we analyse the complete model presented in the previous chapter. We examine the question of the existence of a weak solution of the corresponding initialboundary value problem and consider the question of the uniqueness of the solution. As in the case of many evolutionary problems arising from the physics, the problem consists of a second order parabolic initial boundary value problems and requires the treatment of a quasilinear problem. In order to avoid further complications we have neglected all the mechanical effects (like e.g. shrinking accompanying cooling, stress analysis, etc.).

In order to deepen the analysis of some challenging questions which have arisen, we dedicate Chapter 3 to deepening the mathematical investigation. We concentrate therefore on a sub-problem strictly related to the one examined in Chapter 2. On the domain $Q_{T}=\Omega \times(0, T)$, with $\Omega \subset \mathbb{R}^{N}$ we consider the parabolic problem:

$$
\begin{aligned}
\frac{\partial \theta}{\partial t}-\operatorname{div}(a(x, t) \nabla \theta) & =r(\theta, c) \\
\frac{\partial c}{\partial t}-\operatorname{div}(D(\theta, c) \nabla c) & =0 \\
\frac{\partial \theta}{\partial \nu}+h\left(x, \theta, \theta_{\Gamma}(x, t)\right) & =0 \\
-D(\theta, c) \frac{\partial c}{\partial \nu} & =b(x, t)
\end{aligned}
$$

with prescribed initial conditions. They represent, respectively: heat transfer, carbon diffusion, heat exchange and carbon exchange. We address first the question of existence and $L^{\infty}$-boundness of the solutions. We are particularly interested in the question concerning uniqueness and regularity of the solutions of the system above. The question of uniqueness is well known to be more involved than the one of existence. We are aware of the amount of (also very recent) results concerning this topic, which employ disparate techniques, enabling to treat very general cases in abstract settings, under very weak assumptions (see [15],[18] and references therein). Nevertheless, at present, a general theory covering all the possible cases is not available and every approach presents different advantages and disadvantages. A certain theory usually addresses particular issues and develops specific techniques to that aim. Some drawbacks are always present and render these approaches sometimes inapplicable to concrete cases. For instance, an undesired restriction on the dimension of the spatial domain often occurs.

After these considerations, we consider worthy to develop a technique which is selfcontained and elementary as far as possible, in order to solve this problem. We have 
been able to work also in a three-dimensional setting, which is the relevant case for the applications, with very weak assumptions, which can realistically suit a number of application problems. From this point of view, the present study achieves some progresses in the theoretical analysis on quasilinear parabolic systems, pursuing a new technique - to our knowledge - to treat the question of uniqueness, regularity and continuous dependence on the data for a certain class of partial differential equations systems.

A fundamental tool employed in our approach to the problem are the anisotropic Sobolev spaces, which are found to be particularly adequate in the treatment of equations presenting different regularity in tangential and in normal directions, that is the case of our system with associated third-type boundary conditions. Besides this, we proved a refined version of the Gronwall lemma, that we believe to have some independent interest, to conclude the proof of uniqueness. In the chapter we came to prove a theorem that can be read as a generalisation of some embedding theorems for the classical Sobolev spaces, to anisotropic Sobolev spaces. Also this theorem and its corollaries should be of interest to the readership interested in parabolic systems.

In Chapter 4 we present some simulation work. The simulations, performed with realistic data parameters, have been implemented with a software based on finite elements and cover all the stages of the process and show a possible application of the model in a concrete situation. Finally we comment about possible further developments concerning both the mathematical aspects and applications.

A shorter and unified version of Chapters 1 and 2 has been accepted for publication in [22] and the content of Chapter 3 has been submitted in [32]. 


\section{Chapter 1}

\section{Physical background and modelling}

\subsection{Phase transitions in iron-carbon equilibrium di- agram}

There is a wide choice of textbooks and monographs dealing with iron and steel in the context of physics and engineering. The description of the principles of heat treatment and hardening processes presented in this chapter is based on [3], [7] and on the monograph [48]. Iron is an allotropic metal, that is it can exist in more than one type of lattice structure depending upon temperature.

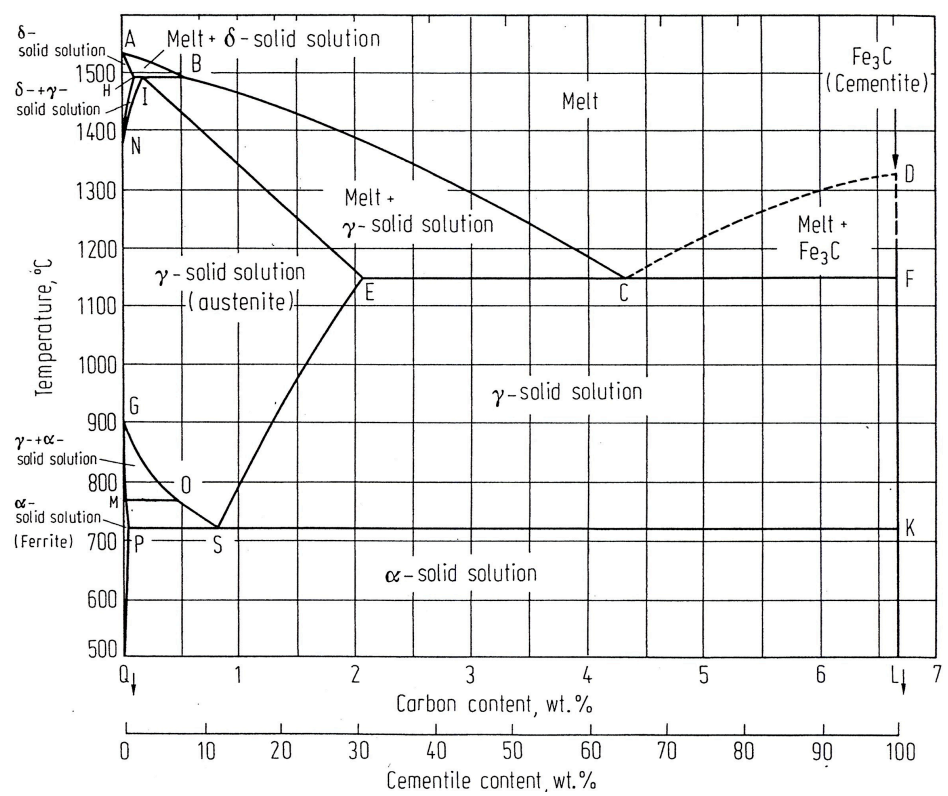

Figure 1.1: The iron-iron carbide equilibrium diagram, (from [48]).

The temperature at which the allotropic changes take place in iron is strongly influenced by alloying elements, the most important of which is carbon. The basis for a description of steel transformations is the diagram shown in Figure 1.1. It represents 
the metastable iron-iron carbide diagram, which is the part of interest, in practical applications, of the iron-carbon phase diagram. It is the part between pure iron and an interstitial compound of iron and carbon, called iron carbide or cementite, (denoted $\mathrm{Fe}_{3} \mathrm{C}$ ) containing $6.67 \%$ carbon by weight. Therefore, this portion of the iron-carbon diagram is called iron-iron carbide equilibrium diagram. It is not a true equilibrium diagram, since equilibrium implies no change of phase with time, whereas the compound iron carbide decomposes into iron and carbon. Although iron-carbide is metastable, its decomposition takes a very long time at room temperature and under conditions of relatively slow temperature changes the system can be considered to cross quasiequilibrium states represented in the iron-carbide diagram.

A phase is a portion of an alloy, physically, chemically, or crystallographically homogeneous throughout, which is separated from the rest of the alloy by distinct bounding surfaces. Phases that occur in iron-iron carbide alloys are austenite ( $\gamma$ phase), ferrite ( $\alpha$ phase), cementite and graphite. They are shown in Figure 1.1. Besides these phases, there are many different structures in steel. On the basis of carbon content it is usual to divide the iron-carbon diagram into two parts. Those alloys containing less than $2 \%$ carbon are known as steel, and those containing more than $2 \%$ carbon are known as cast irons. The interesting region for many heat treatments is the one with carbon content less than $0.8 \%$, the region of hypo-eutectoid steels. Steels with carbon content approximately $0.05-0.15 \%$ are called low-carbon steels. The microscopic structure of steel relies on the configuration of the iron lattice. Depending on temperature, two different lattice structures can occur: a body-centred-cubic (b.c.c.) and a face-centred cubic (f.c.c.) lattice. Above a certain temperature, steel is in the austenitic phase, the so-called $\gamma$-solid solution, an interstitial solid solution of carbon dissolved in f.c.c. iron. In this phase the solubility of carbon in iron is maximal. Below this temperature, the f.c.c. iron lattice is no longer stable. But before the lattice can change its configuration to form a b.c.c. structure, carbon atoms do diffuse, due to the fact that the solubility of carbon in b.c.c. iron is only about $1.4 \%$ of the solubility in f.c.c. iron. The result is a lamellar aggregate of ferrite and cementite, called pearlite, where ferrite is a solid solution of carbon in b.c.c.. Figure 1.2 shows the two different lattice structures.

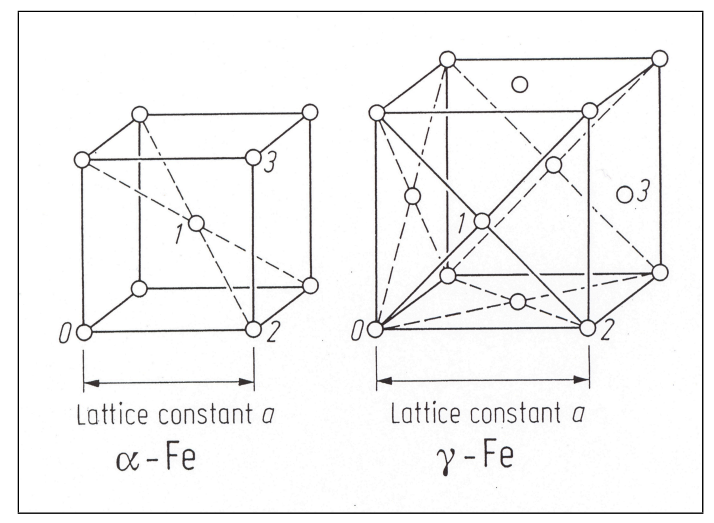

Figure 1.2: Crystal lattice structures of $\alpha$-iron and $\gamma$-iron, (from [48]).

The transformation behaviour associated with various slow cooling rates is called 
diffusion-controlled because the carbon diffusion is dominating. The gamma-to-alpha transformation takes place by a process of nucleation and growth and is time-dependent. Increasing the cooling rate, if a critical cooling rate is overcome, the time for the carbon to diffuse out of solution is insufficient and the structure cannot become b.c.c. while the carbon is trapped in solution. The resultant structure, called martensite, is a solid solution of carbon trapped in a body-centred tetragonal structure.

The microstructure and distribution of different phases are of great importance, due to the fact that each of them possesses different hardness and mechanical properties. Martensite doesn't appear in diagram 1.1 since it is a metastable phase of steel, formed by a transformation of austenite below a certain temperature. It appears in the diagrams that will be shown in the next section.It is important for our aim to note that the difference in carbon solubility mentioned above makes it possible to produce defined microstructures and defined mechanical properties by technical heat treatment.

\subsection{Time-temperature-transformation diagrams}

From the iron-carbon diagram it is possible to derive some important facts regarding the properties of steel undergoing some heating or cooling. The kinetics of the phase transitions depicted in the previous section can be indeed described in an isothermaltransformation (IT) diagram, where temperature is plotted against time. Other names for the same curves are time-temperature-transformation (TTT) curves. These diagrams are constructed according to a standard procedure requiring heat treatment and metallographic experiments on samples. As a result of these experiments, two points can be plotted, namely, the time for the beginning and the time for the end of transformation. The entire experiment is repeated at different critical temperatures until sufficient points are determined to draw a curve showing the beginning of transformation. Portions of these lines are often shown as dashed lines to indicate a high degree of uncertainty.

Each different steel composition has its own TTT curve: an example is depicted in Figure 1.3. However, patterns are generally the same for all steels as far as shape of the curves is concerned. The most outstanding difference in the curves among different steels is the distance between the vertical axis and the nose of the $\mathrm{S}$ curve. This distance between the vertical axis and the nose of the $\mathrm{S}$ curve has a profound effect on how the steel must be cooled to form the hardened structure, martensite. The start of the transformation in the diagram is marked by the line at which $1 \%$ of the new microstructure is formed from the austenite. The curve for complete transformation is drawn for the point at which $1 \%$ of austenite is left. Above the critical temperature $A c_{3}$, austenite is stable. The area on the left of the beginning of transformation consists of unstable austenite. The area on the right of the end of transformation line is the product to which austenite will transform at constant temperature. The area between the beginning and the end of the transformation consists of the microstructures: pearlite $(\mathrm{P})$, ferrite $(\mathrm{F})$, bainite $(\mathrm{B})$ and martensite $(\mathrm{M})$. 


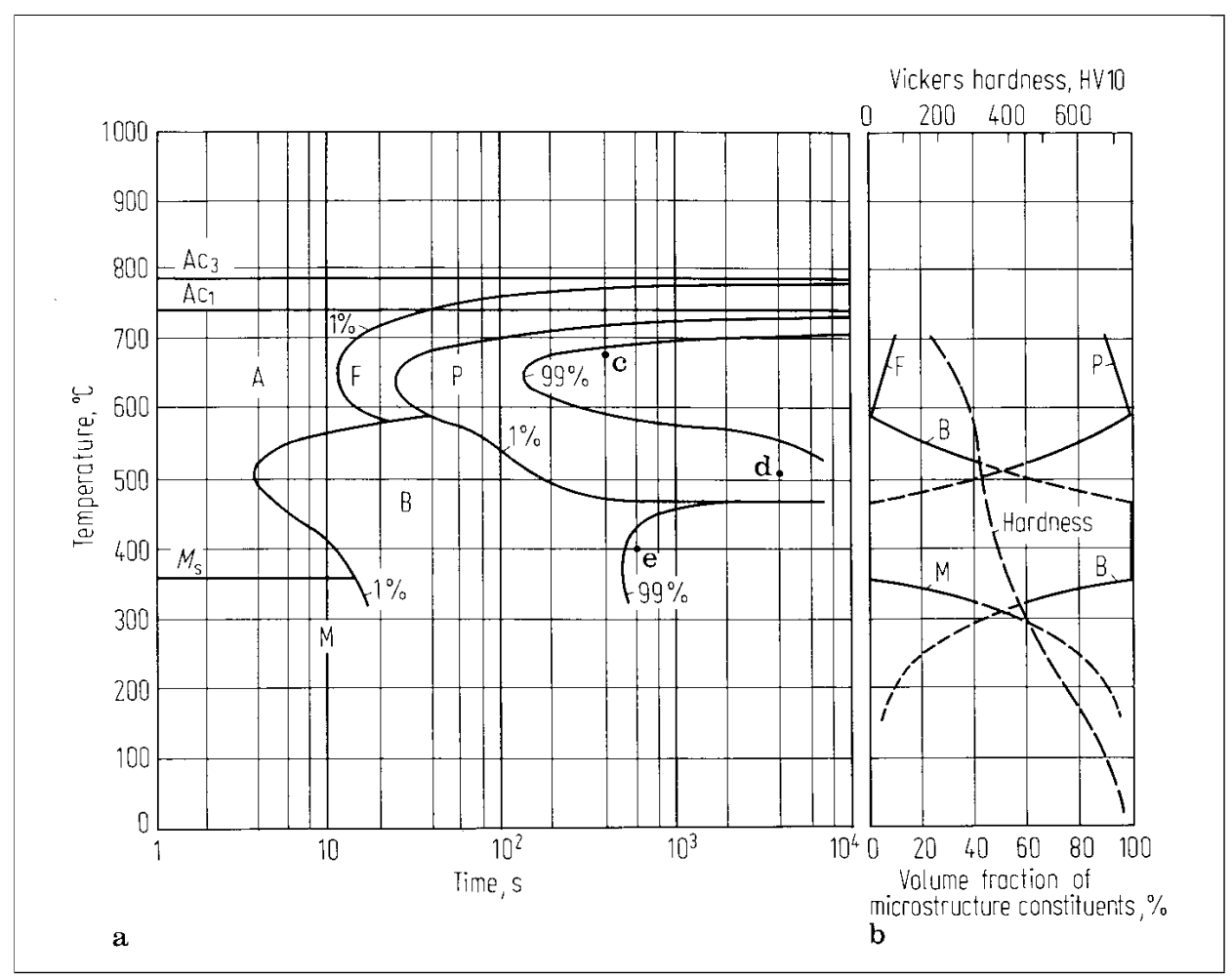

Figure 1.3: (a) Isothermal transformation diagram for the steel $41 \mathrm{Cr} 4$; (b) volume fraction of microstructures (solid lines) and Vickers hardness measured at room temperature (dotted line), (from [48]).

The temperature at which the martensite transformation starts is denoted by $M_{s}$. It is almost impossible to determine experimentally with precision the temperature at which the martensite formation is finished, for this reason we can find very seldom time-temperature-transformation diagrams with the indication of $M_{f}$, but rather with $M_{50}$ or $M_{90}$, denoting respectively the temperature at which the amount of formed martensite is $50 \%$ or $90 \%$.

Phase transformations in steel, during different heat treatments, represent a wide field of investigation. For discussion concerning the material properties of steel in correlation with phase transformations and heat treatment, we refer to [3], [9] and[48].

As the austenite-pearlite transformation is a nucleation and growth process, it is governed by the nucleation rate (the amount of nuclei of the new phase formed per unit time and volume) and by the growth rate of the nuclei. Both of these factors depend on temperature, but in a different way: while the growth rate is high at high temperature and then decreases with decreasing temperature, the nucleation rate increases with decreasing temperature. This behaviour explains the 'nose shape' of the pearlite transformation curves.

Bainite is the product of a transformation which may take place, depending on the type of steel, in the temperature range between the pearlitic and martensitic phase. This additional transformation is shown very distinctly in the TTT diagrams of lowalloyed steels (see for instance Figure 1.3). Bainite is a microstructure made of carbide and martensite. It is generally difficult to recognize it with precision since the bainitic 
transformation presents similar features to the martensitic one and bainite presents a similar aspect to martensite (the german name for bainite 'Zwischenstufengefüge', means 'phase in between' and it is denoted by ZW).

In Figure 1.4, the microstructure of austenite is shown on the right. It is visible that the lower the temperature at which transformation takes place, the higher the hardness. It is also evident that all the structures from the top to the region where marteniste forms are time-dependent, but the formation of martensite is not time-dependent.

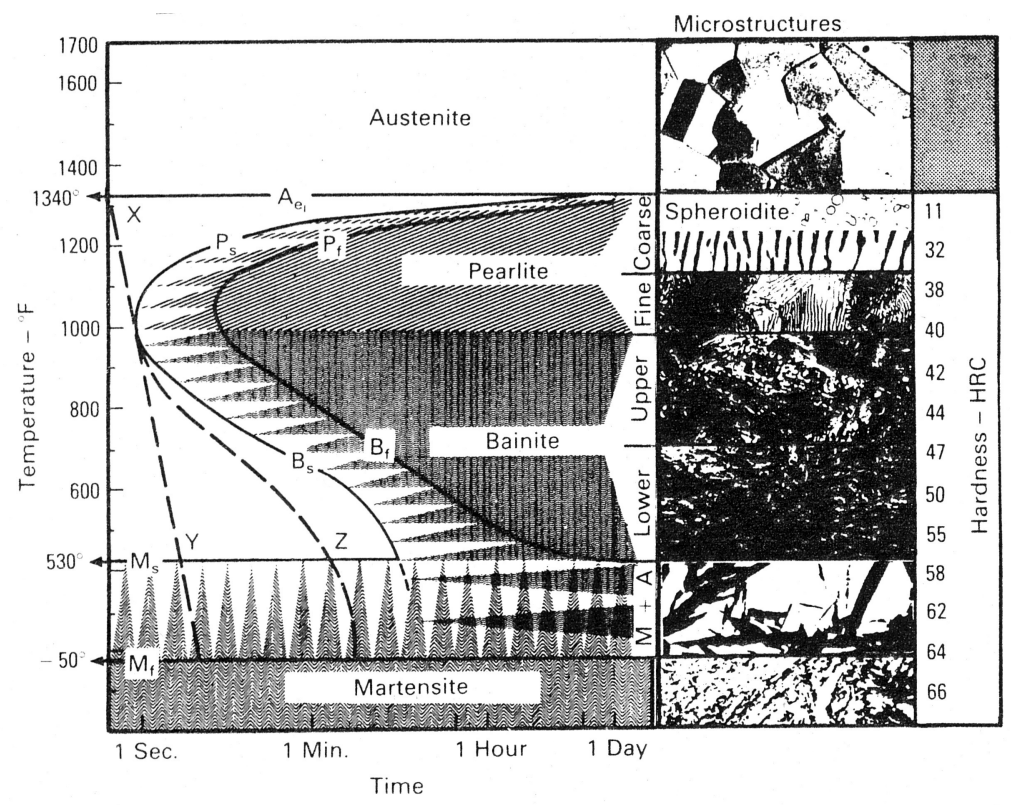

Figure 1.4: TTT diagram for an eutectoid carbon steel $(0.77 \% \mathrm{C})$, (from [7]).

The austenite-martensite transformation is responsible for the outstanding position of steel, because it permits the extreme hardenability of this material. Concerning the martensitic transformation, which is important for the process that we are going to study, we list, below, its main properties.

- The martensitic transformation proceeds only during cooling and ceases if cooling is interrupted. If the steel is held at any temperature below the $M_{s}$, the transformation to martensite will stop and will not proceed again unless the temperature is dropped.

- Martensite can be considered as a transition structure from the unstable austenite phase to the final equilibrium condition of a mixture of ferrite and cementite. The most significant property of martensite is its great hardness; the distortion occurring in the lattice during cooling is its prime reason. Although martensite is harder than the austenite from which it forms, extreme hardness is possible only in steels containing sufficient carbon. The maximum hardness obtainable from steel in the martensitic phase is a function of carbon content only. 
- The martensite transformation of a given alloy cannot be suppressed, nor can the $M_{s}$ temperature be changed by changing the cooling rate. The temperature range of the formation of martensite is characteristic of a given alloy. Many formulas have been experimentally determined for the dependency of $M_{s}$ upon composition of steels. According to [48], one is:

$$
M_{s}(\mathrm{~K})=812-423(\% C)-30.4(\% M n)-17.7(\% N i)-12.1(\% C r)-7.5(\% M o)
$$

The main dependency is clearly upon carbon concentration. The temperature $M_{s}$ is lowered as the carbon content in the austenite increases. Theoretically, the austenite to martensite transformation is never complete, and small amounts of retained austenite will remain even at low temperatures. The transformation of the last traces of austenite becomes more and more difficult as the amount of austenite decreases. The influence of carbon on the $M_{s}$ and $M_{f}$ temperatures is shown in Figure 1.5.

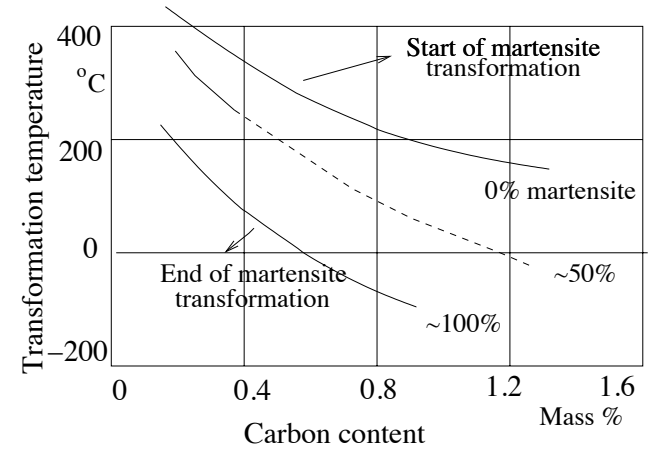

Figure 1.5: Influence of carbon on the martensite formation.

The IT diagram shows the time-temperature relationship for austenite transformation only as it occurs at constant temperature, but most heat treatments involve transformation on continuous cooling. Therefore, for the planning of heat treatments, it is important to predict the (nonisothermal) evolution of the phase fractions. This can be done by means of another type of diagram, the continuous-cooling-transformation (CCT) diagram, which describes the transformation of austenite during countinuous cooling. Figure 1.6 shows a CCT diagram for the steel whose TTT diagram has been previously shown (Fig. 1.3). As for the isothermal transformation, in general the start is defined as the temperature, at which $1 \%$ of the new microstructure has formed. The transformation is completed when only $1 \%$ of the original austenite is left. At each cooling curve these points are connected by smooth curves which represent the CCT diagram. The end of the formation of a microsctructure is not clearly marked if another microstructure follows with decreasing temperature, as in the case of martensite after bainite. The end of the transformation in the martensite range is difficult to detect and therefore is not generally drawn.

Cooling curves are often characterised by the time taken for the specimen to cool from $800^{\circ} \mathrm{C}$ to $500^{\circ} \mathrm{C}$ or from $A c_{3}$ to $500^{\circ} \mathrm{C}$. CCT diagrams must be interpreted by following cooling curves. The start of the austenite transformation and the microstructures 
formed can be read from each cooling curve. Additional information on the volume fractions of the microstructures and the hardness of the specimen at room temperature are plotted as a function of cooling time in Figure 1.6 (b).

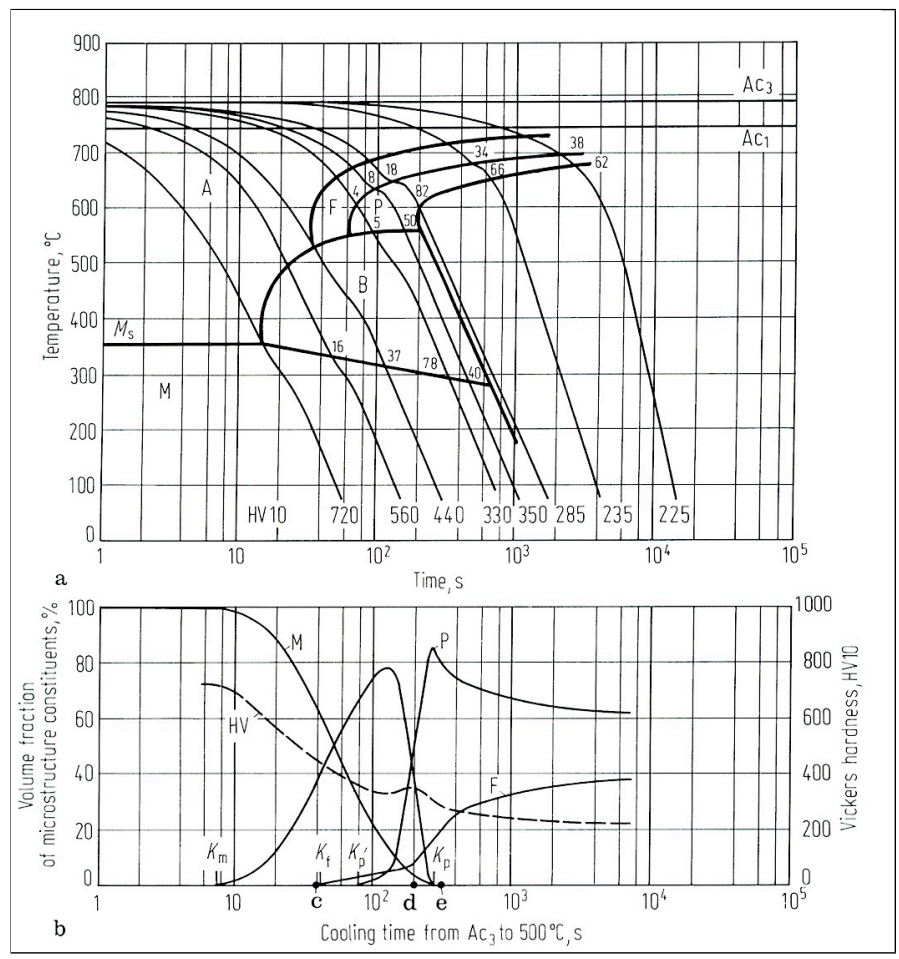

Figure 1.6: (a) Diagram for transformation during continuous cooling (CCT diagram) for the steel $41 \mathrm{Cr} 4$. The numbers on the cooling curves denote the volume fraction of microstructure constituents at room temperature; the numbers at the end of the cooling curves are the Vickers hardness at room temperature. (b) Volume fraction of microstructure constituents (solid lines) and Vickers hardness as function of cooling rate (dotted line) at room temperature, (from [48]).

Considering the IT diagram in relation to the location of the lines of the CCT diagram the 'nose' has been moved downward and to the right by continuous cooling.

The relation between the CCT diagram and the equilibrium diagram is plotted in Figure 1.7. With infinitely slow cooling or heating rate, the CCT diagram is identical to the equilibrium diagram for the composition of the steel. The equilibrium diagram is the limit to the transformation diagrams for infinite transformation time and infinitely slow cooling rate, respectively.

Figure 1.8 shows the relation between time, temperature and carbon content. The cooling curves are not depicted on this diagram for better readability. The line at 0.1 seconds shows the change of the $M_{s}$ temperature depending on the carbon content. 


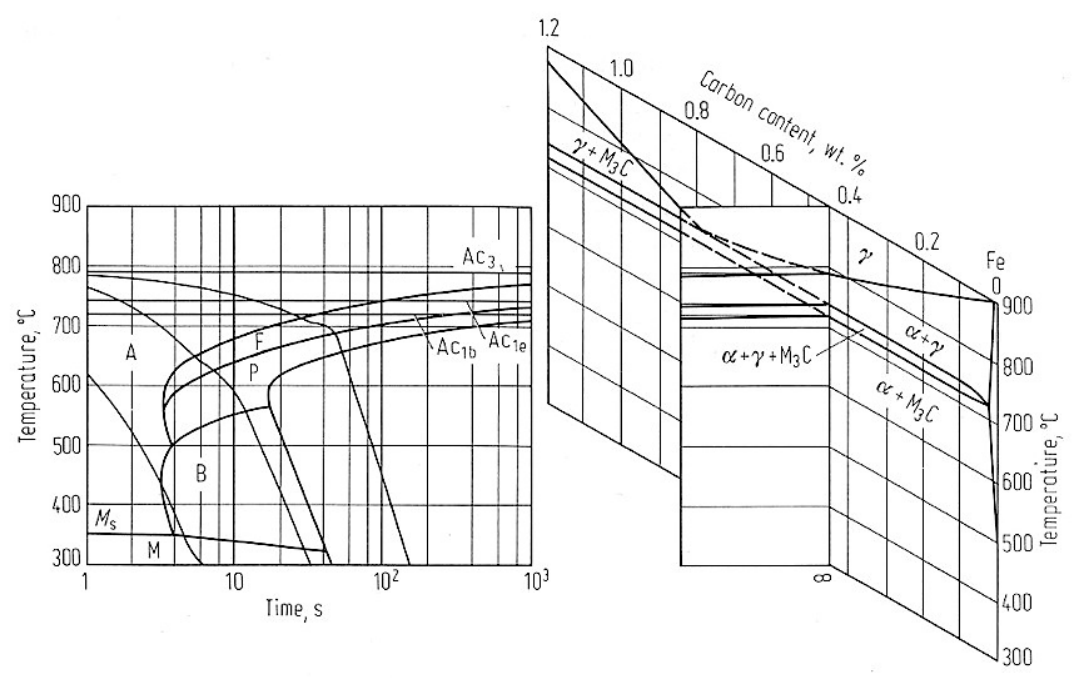

Figure 1.7: Equilibrium diagram of the system iron-carbon (right) as limitation of the CCT diagram of the steel $41 \mathrm{Cr} 4$ (left) with infinite low cooling rate, (from [48]).

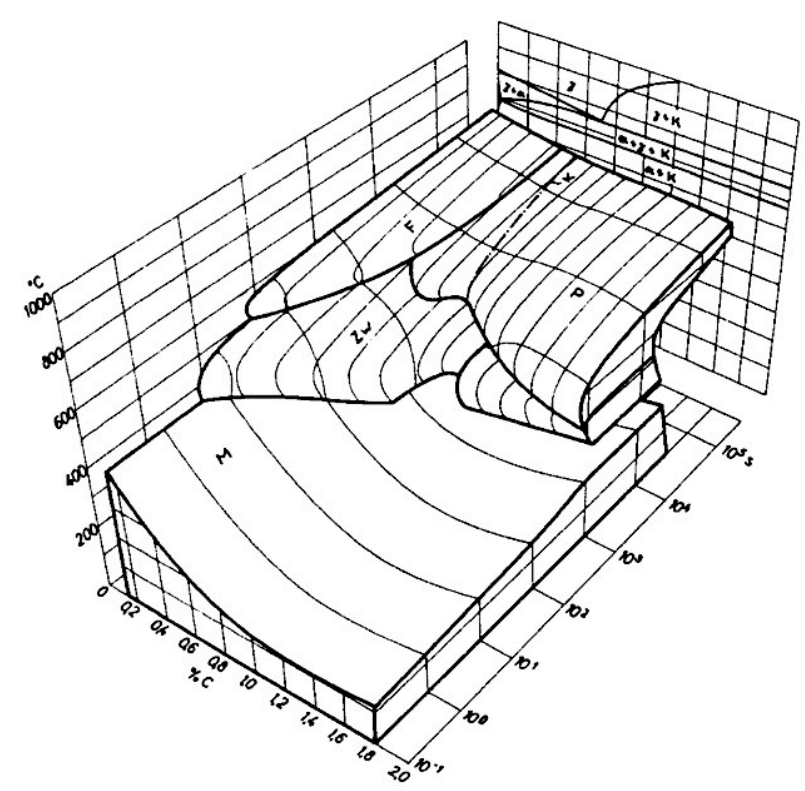

Figure 1.8: Three-dimensional presentation of the transformation characteristic of a $14 \mathrm{NiCr} 14$ steel, for continuous cooling, after austenization at $1023 \mathrm{~K}$ (Symbols: ZW bainite, $\mathrm{M}$ martensite, $\mathrm{P}$ pearlite, $\mathrm{F}$ ferrite), (from [48]).

Since we are interested in steels with carbon content varying from the core to the boundary, therefore diagrams as the one plotted in Figure 1.8 would also be needed in order to follow the transformation at all carbon levels, instead of a single CCT diagram for a steel with fixed carbon content. The cross sections for fixed carbon percentages give CCT diagrams like the one plotted in the left part of Figure 1.7. However, we 
note that such three-dimensional diagrams are very rare in literature and, as it will be shown in Chapter 4, other strategies are needed to determine the parameters entering in the equations describing the phase transitions.

\subsection{Heat treatment of steel: case hardening}

According to a definition given in [3], heat treatment of steel is a combination of heating and cooling operations, applied to a metal or alloy in the solid state in a way that will produce the desired properties.

All basic heat-treating processes for steel involve the transformation or decomposition of austenite. The nature and appearance of these transformation products determine the physical and mechanical properties of any given steel. All the different heat treatment processes consist of the following three stages, possibly cyclically repeated in different sequences:

1) heating of the material to some temperature in or above the critical range in order to form austenite;

2) holding the temperature for a certain period of time;

3) cooling, usually to room temperature.

The temperature and time for the various stages depend on the desired effect. During heating and cooling there exist temperature gradients between the outside and interior portion of the material, whose magnitude depends on the size and geometry of the workpiece.

All the heat treatments are associated with phase transformations in steel and indeed the phase transformations are the reason of the feasibility of the treatment itself. Generally, phase transformations may lead to mechanical deformation of the workpiece. Because of the mathematical complexity of the model that we are going to introduce, we will not include mechanical behaviour (e.g. thermo-elasticity, transformation induced plasticity) in our model. Therefore, distortion, in particular that related to the case hardening, can not be described by the model that we are going to propose.

This thesis is concerned with a particular kind of heat treatment, named case hardening, which is the production of parts that have hard, wear-resistant surfaces, but softer and/or tougher cores. It can be accomplished by two distinct methods. One approach is the use of a grade of steel that already contains sufficient carbon to provide the required surface after heat treatment (as flame or induction hardening). The surface areas requiring the higher hardness are then selectively heated and quenched. The second method is the use of a steel that is not normally capable of being hardened to the desired degree, altering the composition of the surface layers by diffusion so that it either can be hardened during processing.

For practical purposes, case hardening of the second type can be broadly classified into four groups: carburizing, carbonitriding, nitriding and nitrocarburizing. Of these processes, carburizing is by far the most widely used. 


\subsection{Modelling of gas carburizing}

\subsubsection{Carbon diffusion in austenite}

The primary object of carburizing is to provide a hard, wear-resistant surface with surface residual compressive stresses, that results in an improvement in the life of ferrous engineering components. In carburizing, austenitized ferrous metals are brought into contact with an environment of sufficient carbon potential to cause absorption of carbon at surface, creating, by diffusion, a concentration gradient between the surface and the interior of the metal.

Carburizing may be done in a gaseous environment (gas carburizing), a liquid salt bath (liquid carburizing), or with all the surfaces of the workpiece covered with a solid carbonaceous compound (pack carburizing). Regardless of the process, the objective of carburizing is to start with a relatively low-carbon steel $(0.2 \% \mathrm{C})$ and to increase the carbon content in the surface layers, resulting in a high-carbon, hardenable steel on the outside with a gradually decreasing carbon content to the underlying layers. Process control to keep the desired carbon level is an important part of any carburizing process. Usually, although not always, approximately $0.8 \%$ is desired. Among the carburizing variants, the commercially most important is called gas carburizing, in which source of carbon is a carbon-rich furnace atmosphere: we will focus on this method. It is more effective, with deeper and higher carbon content cases obtained more rapidly and more adaptable for mass production than other carburizing processes.

During the process, the carbon-bearing atmosphere can be continuously replenished so that a high carbon potential can be maintained. A wide variety of furnaces are used for gas carburizing; selection of furnace type depends largely on workpiece shape and size, total production required and production flow.

It must be said that carburizing is a distinctly separate operation, taking place at constant temperature, high enough to have an austenitic microstructure, followed in most case by quenching, to form a martensitic microstructure, which produces the desired hardening effect. We will present a model for the complete process, including carburization and cooling.

The quality of the carburized samples is determined on the basis of the hardness and case depth required for a particular application and of compliance with given specifications and tolerances. Therefore it is necessary to understand the mechanism of carbon transfer and to accurately predict the carbon concentration profile and the case depth during heat treatment process.

The process of carburization can be divided in: transport of carbon to surface, reaction on the surface and diffusion of the carbon in the solid. The model we are going to present is a phenomenological description at the macroscopic level. Temperature and carbon concentration, in weight percent, are the physical variables playing the main role during carburization.

For an insight into aspects like atmosphere composition and kinetics of carbon transfer refer, for example, to [48].

The mechanisms of carbon transfer during carburizing is shown in Figure 1.9, where the most important physical parameters involved in the process are indicated: the mass 
transfer coefficient $(\beta)$, defining carbon atoms flux $(j)$ from the atmosphere through the steel surface, and the coefficient of carbon diffusion in steel $(D)$ at austenitizing temperature.

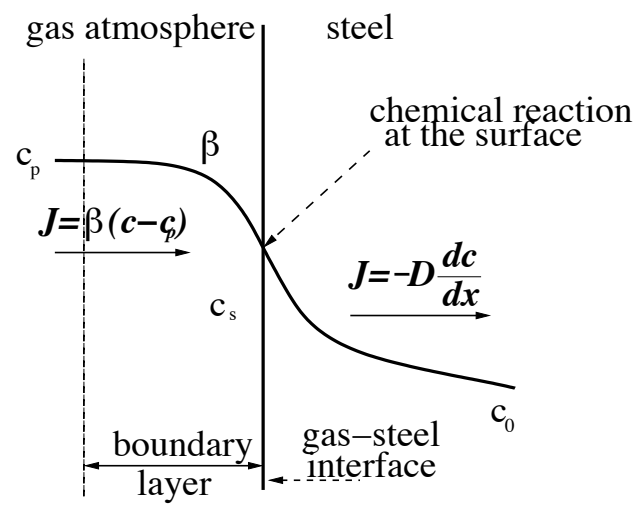

Figure 1.9: Schematic representation of carbon transport in carburizing.

The mass transfer coefficient, $\beta$, in the gas phase controls the rate of carbon uptake during the initial stage of carburizing. Several efforts to measure $\beta$ have been made under variable temperature, atmosphere composition and carburizing potential. According to [16], [44], [45], its value ranges from $1 \cdot 10^{-9}$ to $2 \cdot 10^{-4} \mathrm{~cm} / \mathrm{s}$ at temperatures between $800^{\circ} \mathrm{C}$ and $1000^{\circ} \mathrm{C}$; nevertheless in many circumstances, it is not significant error assuming $\beta$ constant.

The ability of the furnace atmosphere to supply carbon to the heated steel is called carbon potential, denoted with $c_{p}$ (see Figure 1.9) and is defined as the percent carbon (by weight) in a piece of steel which has achieved thermodynamic equilibrium with the atmosphere. For example, if a part with $0.6 \%$ carbon neither takes carbon from the furnace atmosphere, nor gives up carbon to it, the atmosphere is said to have a carbon potential of $0.6 \% \mathrm{C}$. In such atmosphere, a part with $0.5 \% \mathrm{C}$ would take carbon from the atmosphere (i.e. would be carburized) and a part with $0.7 \% \mathrm{C}$ would be decarburized, (from [51]).

According to Fick's first law, the flow of substance is proportional to the concentration gradient

$$
j=-D(\theta, c) \nabla c,
$$

where the diffusivity $D=D(\theta, c)$ is a function of the concentration $c$ and the temperature $\theta$.

The unsteady process of diffusion of carbon in $\gamma$-iron (austenite) is described by the following parabolic equation

$$
\frac{\partial c}{\partial t}-\operatorname{div}(a D(\theta, c) \nabla c)=0
$$

The factor $a$, denoting the austenite fraction, in front of the diffusion coefficient $D(\theta, c)$ reflects the fact that enrichment with carbon only takes place in the austenite phase. 
Now the boundary conditions must be assigned. Since the difference in carbon potential between the surface and the workpiece provides the driving force for carbon diffusion, we prescribe the following boundary condition:

$$
-a D(\theta, c) \frac{\partial c}{\partial \nu}=\beta(\theta)\left(c-c_{p}\right)
$$

where $\beta$, the mass transfer coefficient, controls the rate at which carbon is absorbed by the steel during carburizing and $c_{p}$ is the carbon potential in the furnace. $\frac{\partial c}{\partial \nu}$ denotes the outward normal derivative. There are many formulas in literature for the carbon diffusivity in austenite, $D(\theta, c)$, according to the kinetic and thermodynamic behaviour of carbon in austenite. We refer to Chapter 4 for some comments about this coefficient.

\subsubsection{Phase transformations}

In this study, we take into account the phase transitions from austenite to pearlite and from austenite to martensite. In other words we assume that all the austenite present at the beginning of the transformation will be completely turned into pearlite and martensite, at the end. However, a more elaborate model accounting for all the phase transitions occurring during the heat treatment of steel can be found in [23], [50] and references therein.

Since the densities of the phases in steel differ only very little from each other (for fixed temperature), we do not distinguish between mass and volume fractions of the phases. Therefore, in the following, we will refer only to the 'phase fractions'.

As already mentioned before, in this study, we do not consider the heating stage and assume that cooling takes place from the high temperature phase austenite with phase fraction $a$ to two different product phases, pearlite with fraction $p$ and martensite with fraction $m$.

The model that we are going to present is a mathematical description of the phenomenologically observed phase transitions in steel. The basic assumption for our approach is that all the information about the evolution of phase transitions in a specific steel is contained in the isothermal and non-isothermal time-temperaturetransformation diagrams. We propose the simplest phenomenological model able to describe our situation, that was developed, in the context of heat treatment problems, in a series of papers ([19], [20], [23]), to which we refer for an exhaustive discussion about modelling and for comparisons with other possible models. We consider the following equations to describe the evolution of $p$ and $m$ :

$$
\begin{aligned}
p_{t} & =(1-p-m) g_{1}(\theta, c) \\
m_{t} & =[\bar{m}(\theta, c)-m]_{+} g_{2}(\theta, c) \\
p(0) & =0 \\
m(0) & =0
\end{aligned}
$$

where $c$ is the concentration of carbon. Here the bracket []$_{+}$denotes the positive part function $[x]_{+}=\max \{x, 0\}$ and the subscript $t$, as usual for PDEs, denotes the 
derivative with respect to time, which can be found in literature denoted with the dot (e.g. $\dot{p}$ instead of $p_{t}$ ).

While the growth rate of pearlite $p_{t}$ is assumed to be proportional to the remaining austenite fraction, the rate of martensite growth $m_{t}$ is zero if $m$ exceeds either the non-perlitic fraction $1-p$, or the threshold $\bar{m}$, depending on both temperature and carbon concentration. The quantity $\bar{m}(\theta, c)$ represents the maximum attainable value of martensite fraction and is defined as

$$
\bar{m}(\theta, c)=\min \left\{m_{K M}(\theta, c) ; 1-p\right\} .
$$

The function $m_{K M}$ describes the volume fraction of martensite according to the Koistinen-Marburger formula [29], i.e.,

$$
m_{K M}(\theta, c)=\left(1-e^{-c_{k m}(c)\left(M_{s}(c)-\theta\right)}\right) H\left(M_{s}(c)-\theta\right),
$$

where $H$ is the heaviside function, $c_{k m}$ and $M_{s}$ here are parameters depending on the carbon concentration and can be drawn from the respective TTT diagram. If during some stage of the heat treatment $\theta \geq M_{s}$, owing to the heaviside function, we have $m_{t}=0$, whence no martensite is produced during this stage. $c_{k m}$ is a constant reflecting the transformation rate and varying with the steel composition. We note that, since $m_{t}=0$, the irreversibility of the martensite transformation is incorporated in the model. The functions $g_{1}$ and $g_{2}$ are positive given functions that can be identified from the time-temperature-transformation diagrams described before. In Chapter 4 we will illustrate how this identification can be done for a specific steel. The final hardness is obtained by accumulating the contributions of the different phases formed along cooling.

\subsubsection{Heat transfer}

With the purpose of obtaining the temperature equation governing the whole process, we start considering the energy balance. For an incompressible, rigid body, it holds (see for instance [10]) the following balance of energy

$$
\rho \frac{\partial e}{\partial t}+\operatorname{div} \mathrm{q}=0
$$

where $e$ is the specific internal energy, $q$ is the heat flux and $\rho$ is the density. Considering the whole process, we assume that the specific internal energy has the form $e=\hat{e}(\theta, p, m)$, with a differentiable material function $\hat{e}$. Applying the Fourier's law we obtain

$$
\mathrm{q}=-k(\theta) \nabla \theta,
$$

where $k(\theta)$ is the heat conductivity of the material and we derive the following equation for the temperature

$$
\rho \frac{\partial \hat{e}}{\partial \theta} \frac{\partial \theta}{\partial t}+\rho \frac{\partial \hat{e}}{\partial p} \frac{\partial p}{\partial t}+\rho \frac{\partial \hat{e}}{\partial m} \frac{\partial m}{\partial t}-\operatorname{div}(k(\theta) \nabla \theta)=0 .
$$


We denote the partial derivatives

$$
\frac{\partial e}{\partial \theta}=\hat{\alpha}(\theta, p, m), \quad \frac{\partial e}{\partial p}=-\hat{L}_{p}(\theta, p, m), \quad \frac{\partial e}{\partial m}=-\hat{L}_{m}(\theta, p, m) .
$$

Concerning the material functions $\hat{\alpha}, \hat{L}_{p}, \hat{L}_{m}$, we follow the modelling of [23], where they only depend on $\theta: \hat{\alpha}(\theta, p, m):=\alpha(\theta), \hat{L}_{p}(\theta, p, m):=L_{p}(\theta), \hat{L}_{m}(\theta, p, m):=L_{m}(\theta)$. Here $\alpha(\theta)$ represents the specific heat and $L_{p}(\theta)$ and $L_{m}(\theta)$ denote latent heats of the austenite-pearlite and the austenite-martensite phase changes, respectively. They are related to the enthalpy of the transformation and to the rate of transformation. Overall, we have obtained that the heat equation during the whole process can be written as

$$
\rho \alpha(\theta) \frac{\partial \theta}{\partial t}-\operatorname{div}(k(\theta) \nabla \theta)=\rho L_{p}(\theta) p_{t}+\rho L_{m}(\theta) m_{t}
$$

The heat source stems from the latent heats due to the phase transformations occurring during cooling. During the phase transitions from austenite to pearlite and from austenite to martensite, heat is released, therefore the functions $L_{p}(\theta)$ and $L_{m}(\theta)$ are always positive. Since the terms $p_{t}, m_{t}$, given through the formulas (1.4.1a),(1.4.1b) are positive as well, we can immediately see that the right-hand side of equation (1.4.2) is a positive quantity. We pass now to assigning the initial condition and the boundary condition, neglecting conduction and radiation effect:

$$
\begin{aligned}
-k(\theta, c) \frac{\partial \theta}{\partial \nu} & =h\left(\theta-\theta_{\Gamma}\right) \\
\theta(x, 0) & =\theta_{0}(x) .
\end{aligned}
$$

Here $\theta_{\Gamma}$ is the external temperature, $\theta_{0}(x)$ is the temperature at the beginning of the process and $h$ is the heat transfer coefficient. Since we do not take into account mechanical effects, we assume the density $\rho$ to be constant.

In conclusion, the evolution of temperature during the process can be described by the non-linear problem:

$$
\begin{aligned}
\rho \alpha(\theta) \frac{\partial \theta}{\partial t}-\operatorname{div}(k(\theta) \nabla \theta) & =\rho L_{p}(\theta) p_{t}+\rho L_{m}(\theta) m_{t} \\
-k(\theta) \frac{\partial \theta}{\partial \nu} & =h\left(\theta-\theta_{\Gamma}\right) \\
\theta(x, 0) & =\theta_{0}(x) .
\end{aligned}
$$

\subsubsection{Complete model of gas carburizing}

We collect now the equations from the previous sections. Let $\Omega \subset \mathbb{R}^{3}$ be an open bounded set with boundary $\partial \Omega$ and $Q_{T}:=\Omega \times(0, T)$ the corresponding parabolic cylinder, where $T$ represents the end time of the process. We obtain the following initial-boundary value problem. 


$$
\begin{aligned}
\rho \alpha(\theta) \frac{\partial \theta}{\partial t}-\operatorname{div}(k(\theta) \nabla \theta)=\rho L_{p}(\theta) p_{t}+\rho L_{m}(\theta) m_{t} & \text { in } Q_{T} \\
\frac{\partial c}{\partial t}-\operatorname{div}((1-p-m) D(\theta, c) \nabla c)=0 & \text { in } Q_{T} \\
p_{t}=(1-p-m) g_{1}(\theta, c) & \text { in } Q_{T} \\
m_{t}=[\min \{\bar{m}(\theta, c) ; 1-p\}-m]_{+} g_{2}(\theta, c) & \text { in } Q_{T} \\
-k(\theta) \frac{\partial \theta}{\partial \nu}=h\left(\theta-\theta_{\Gamma}\right) & \text { on } \partial \Omega \times(0, T) \\
-(1-p-m) D(\theta, c) \frac{\partial c}{\partial \nu}=\beta(\theta)\left(c-c_{p}\right) & \text { on } \partial \Omega \times(0, T) \\
\theta(x, 0)=\theta_{0}(x) & \text { in } \Omega \\
c(x, 0)=c_{0}(x) & \text { in } \Omega \\
p(0)=0 & \text { in } \Omega \\
m(0)=0 & \text { in } \Omega
\end{aligned}
$$

The central part of this thesis is the analytical investigation of the mathematical model presented above.

It permits to evaluate the effects of all the possible interactions between carbon diffusion, heat treatment and growth of phases in every stage of the process. Actually it would be capable to describe more general situations, for instance any other process composed of many cycles of heating and cooling, associated to the diffusion of a substance and to the evolution of temperature. Motivated by its relevance in industrial applications, we decided to concentrate on the analysis of the process of gas carburizing.

In the previous sections, we were concerned with the physical phenomena occurring during case hardening. For clarity, now we summarise the process schedule and fix it in a schematic representation (Figure 1.10).

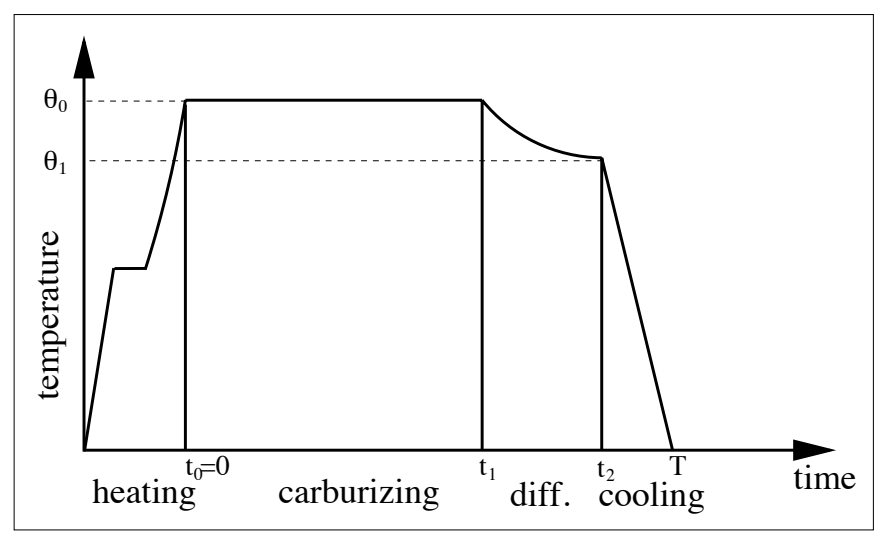

Figure 1.10: Schematic carburizing cycle.

We distinguish three characteristic times: the beginning of the carburization process $t_{0}$, the end of the carburization period $t_{1}$, the end of the diffusion period $t_{2}$ and the 
end of the cooling $T$.

First, the workpiece is heated up in a neutral atmosphere to a predetermined temperature $\theta_{0}$ in the range from 1100 to 2150 . During heating, steel is transformed to the austenite phase. Note that this period is not covered by the system (1.4.6)-(1.4.15). Then a carbon rich gas such as propane, butane or methane is injected into the furnace. The workpiece is held at this temperature, to allow for the diffusion of carbon into the case. During this stage of the process, no phase transformation takes place, since the workpiece remains at the austenitizing temperature, well above the temperature where the formation of other phases can start. This fact is clearly observable in Figure 1.4. After the main carburizing stage, a slow diffusion period is sometimes allowed, through which the workpiece is brought to a lower specified temperature $\theta_{1}$, whilst the carbon potential is lowered and the furnace is slowly cooled down. This diffusion step is used to remove the marked fluctuation in carbon concentration in the near-surface layer, which arises in the process of initial saturation. By allowing such a diffusion period, moreover, the surface carbon content can be reduced to any desired value. Again, in this further diffusive stage, the workpiece remains at a temperature high enough to prevent the formation of new phases, namely in the region where only the presence of austenite is possible.

After the diffusion period, the components are quenched to obtain the required hardness and wear resistance on the surface. The duration of quenching is few minutes. Depending on the required depth of carburization, the total time of the process varies from one hour to several hours.

Of course for each different stage in which the whole process can be divided, as shown in Figure 1.10, we can consequently specify the coefficients involved in the boundary conditions:

- Stage 1: carburizing in a furnace, hence $\beta \geq 0$ and $h=0$, since the carburizing takes place at constant temperature.

- Stage 2: diffusion period, with $\beta \geq 0, c_{p}$ smaller than in Stage 1 and $h \geq$ 0 , serving as a linearized radiation law (a non linearized radiation law will be separately considered in Chapter 3 ).

- Stage 3: quenching with $\beta=0$ and $h \geq 0$. During quenching the workpiece is taken out from the furnace and therewith from the carbon-reach atmosphere. This happens below the temperature necessary for the diffusion of carbon (previously named $\theta_{1}$ ), therefore both further carburization and decarburization can be neglected. Hence we can assume $\beta$ to be zero. 


\section{Chapter 2}

\section{Analysis of the complete model}

\subsection{Problem statement and strategy}

This chapter is devoted to the analysis of the system (1.4.6)-(1.4.15), that we rewrite below for clarity:

$$
\begin{aligned}
\rho \alpha(\theta) \frac{\partial \theta}{\partial t}-\operatorname{div}(k(\theta) \nabla \theta)=\rho L_{p}(\theta) p_{t}+\rho L_{m}(\theta) m_{t} & \text { in } Q_{T} \\
\frac{\partial c}{\partial t}-\operatorname{div}((1-p-m) D(\theta, c) \nabla c)=0 & \text { in } Q_{T} \\
p_{t}=(1-p-m) g_{1}(\theta, c) & \text { in } Q_{T} \\
m_{t}=[\min \{\bar{m}(\theta, c) ; 1-p\}-m]_{+} g_{2}(\theta, c) & \text { in } Q_{T} \\
-k(\theta) \frac{\partial \theta}{\partial \nu}=h\left(\theta-\theta_{\Gamma}\right) & \text { on } \partial \Omega \times(0, T) \\
-(1-p-m) D(\theta, c) \frac{\partial c}{\partial \nu}=\beta\left(c-c_{p}\right) & \text { on } \partial \Omega \times(0, T) \\
\theta(x, 0)=\theta_{0}(x) & \text { in } \Omega \\
c(x, 0)=c_{0}(x) & \text { in } \Omega \\
p(0)=0 & \text { in } \Omega \\
m(0)=0 & \text { in } \Omega
\end{aligned}
$$

The model accounts for the coupling of temperature, phase transitions and carbon diffusion. We will prove existence and uniqueness of a weak solution of system (2.1.1)(2.1.10).

The proof of existence is carried out using a nested fixed point argument. We divide the proof in three steps. The first is a preliminary lemma concerning the ODE system $(2.1 .3),(2.1 .4),(2.1 .9),(2.1 .10)$ only, for $\theta$ and $c$ prescribed. It is the case of a ODE system with discontinuous right-hand side, where the derivatives are defined almost everywhere. We will show that the conditions of the Charathéodory existence theorem are satisfied and, afterwards, that the solution is unique.

The second step is the coupling of the ODE system with the temperature equation, which gives a solution $p, m, \theta$ depending on $c$. The third and last step is the further 
coupling with the equation for $c$. To prove existence of a solution for the whole system, we proceed applying twice the Schauder fixed point theorem. To obtain uniqueness we must consequently develop an ad hoc technique.

\subsection{Assumptions and main results}

During this chapter we assume that $\Omega \subset \mathbb{R}^{3}$ and $\partial \Omega$ is its $C^{2}$-boundary and we denote $Q_{T}:=\Omega \times(0, T)$ the corresponding space-time cylinder. During this and the following chapter we make use of the following notations:

- $W^{1, \infty}\left(0, T ; L^{\infty}(\Omega)\right)=\left\{v \in L^{\infty}\left(0, T ; L^{\infty}(\Omega)\right): v_{t} \in L^{\infty}\left(0, T ; L^{\infty}(\Omega)\right)\right\}$.

- $W_{p}^{r, s}\left(Q_{T}\right)=L^{p}\left(0, T ; W_{p}^{r}(\Omega)\right) \cap W_{p}^{s}\left(0, T ; L^{p}(\Omega)\right)$.

- We denote by $V$ the space $W_{2}^{1}(\Omega)$ and by $V^{*}$ the space $\left(W_{2}^{1}(\Omega)\right)^{*}$.

$W(0, T)=\left\{v \in L^{2}(0, T ; V): v_{t} \in L^{2}\left(0, T ; V^{*}\right)\right\}$, endowed with the norm

$$
\|v\|_{W(0, T)}=\left(\int_{0}^{T}\left(\|v(t)\|_{V}^{2}+\left\|v^{\prime}(t)\right\|_{V^{*}}^{2}\right) d t\right)^{\frac{1}{2}}
$$

The derivative of $u(x, t)$ with respect to $t$ will be denoted, depending on the circumstances, with $\frac{\partial u}{\partial t}$ or with $u_{t}$.

Throughout the chapter we will make use of the following assumptions:

(A1) $\rho$ and $k$ are positive constants.

(A2) $\alpha \in C(\mathbb{R})$ and there exist positive constants $\alpha_{0}, \alpha_{1}$ such that $\alpha_{0} \leq \alpha(\cdot) \leq \alpha_{1}$. $L_{p}, L_{m} \in L^{\infty}(\mathbb{R})$ and they are Lipschitz-continuous.

(A3) $\theta_{\Gamma}$ is a positive constant. $h \in W_{2}^{2}(\partial \Omega)$ with $h(x) \geq 0$ a.e. in $\partial \Omega$. $\theta_{0} \in W_{2}^{2}(\Omega)$, $\theta_{\Gamma} \geq \theta_{0}$.

(A4) $g_{1}, g_{2}$ are Lipschitz-continuous in both arguments and there exist positive constants $\gamma_{1}, \gamma_{2}$ such that $0 \leq g_{1}(\theta, c) \leq \gamma_{1}, \quad 0 \leq g_{2}(\theta, c) \leq \gamma_{2}, \quad \forall \theta, c \in \mathbb{R}$.

(A5) $\bar{m}$ is Lipschitz-continuous satisfying $\bar{m}(\theta, c) \in[0,1]$ for every $\theta, c \in \mathbb{R}$.

(A6) $D(\theta, c)$ is Lipschitz-continuous in both arguments and there are positive costants $\gamma_{3}, \gamma_{4}$ such that $\gamma_{3} \leq D(\theta, c) \leq \gamma_{4}, \forall \theta, c \in \mathbb{R}$.

(A7) $c_{p}$ is a positive constant. $\beta \in L^{\infty}(\partial \Omega)$ with $\beta \geq 0$ a.e. in $\partial \Omega . c_{0} \in L^{2}(\Omega)$ 
We are going to prove that, under the hypothesis above, the considered problem has a weak solution.

Theorem 2.2.1 (Existence of a weak solution). Assume (A1)-(A7), then there exists a weak solution $(\theta, c, p, m)$ to problem (2.1.1)-(2.1.10) such that $\theta \in W_{2}^{2,1}\left(Q_{T}\right), c \in$ $W(0, T), p, m \in W^{1, \infty}\left(0, T ; L^{\infty}(\Omega)\right)$.

The following proposition is also important from the physical point of view, since it provides a uniform bound on the temperature and on the carbon concentration, as we can expect from the process observation.

Proposition 2.2.2. Assume (A1)-(A7). Moreover assume that $\theta_{0} \in L^{\infty}(\Omega)$ and there exist positive constants $C_{1}, C_{2}$ such that $C_{1} \leq c_{p} \leq C_{2}$ in $\partial \Omega \times(0, T)$ and $C_{1} \leq c_{0} \leq C_{2}$ a.e. in $\Omega$. Then

$$
\begin{gathered}
\theta_{\Gamma} \leq \theta \leq \theta_{0} \quad \text { a.e in } Q_{T} \\
C_{1} \leq c \leq C_{2} \quad \text { a.e. in } Q_{T} .
\end{gathered}
$$

Theorem 2.2.3 (Uniqueness). Suppose that (A1)-(A7) are satisfied. Assume moreover that $\alpha$ is constant, $D=D(\theta), h, \beta \in W_{5}^{1}(\partial \Omega), \theta_{0}, c_{0} \in W_{5}^{2}(\Omega)$. Then the solution to (2.1.1)-(2.1.10) is unique.

Remark 1. (a) Concerning Theorem 2.2.1: the regularity assumptions on the boundary and initial values could be weakened. To avoid unnecessary technicalities we assumed $\theta_{\Gamma}$ and $c_{p}$ to be constants, but they could be in fact functions of space and time, according, for instance, to [37].

(b) Concerning Proposition 2.2.2: also in this case some weaker assumptions might be sufficient. Regarding $\theta_{\Gamma}$ and $c_{p}$, it is indeed sufficient their boundedness, but we preferred to keep the setting used for the existence theorem.

\subsection{Proof of existence}

As already mentioned, the proof is carried out using a nested fixed point argument. The first step is a preliminary lemma concerning the ODE system (2.1.3),(2.1.4) only, for $\theta$ and $c$ prescribed. The second step is the coupling of the ODE system and the temperature equation, which gives a solution $p, m, \theta$ depending on $c$ and the third is the further coupling with the equation for $c$. We begin with considering the initial value problem

$$
\begin{aligned}
z_{t} & =f(z, \theta, c) & & \text { in } Q_{T} \\
z(0) & =0 & & \text { in } \Omega
\end{aligned}
$$

where $z=(p, m)^{T}$ and $f=\left(f_{1}, f_{2}\right)^{T}$ denotes the right-hand side of (2.1.3),(2.1.4). The system of ODE's is meant as ODE with value in a Banach space, therefore, writing $z(x, t)$ with an abuse of notation, we mean $z(t)(x)$. 
Lemma 2.3.1. Under the assumptions (A4),(A5) the following statements are valid:

(a) For every $\theta, c \in L^{2}\left(Q_{T}\right)$ problem (2.3.1) has a unique solution $z$ such that $p \geq 0$, $m \geq 0$ and

$$
\||z|\|_{W^{1, \infty}\left(0, T ; L^{\infty}(\Omega)\right)} \leq M
$$

for a constant $M$ independent of $\theta$ and $c$. Moreover, there exists a constant $c_{T}$ such that

$$
0 \leq p(x, t)+m(x, t) \leq c_{T}<1 \quad \text { for a.e. }(x, t) \text { in } Q_{T} .
$$

(b) There are constants $M_{1}, M_{2}>0$ such that for every $\theta_{1}, \theta_{2}, c_{1}, c_{2} \in L^{p}\left(Q_{T}\right)$, for almost all $t \in(0, T)$ and all $p \geq 2$ we have

$$
\left\|\left|z_{1}(t)-z_{2}(t)\right|\right\|_{W^{1, p}(\Omega)}^{p} \leq M_{1} \int_{0}^{t}\left\|\theta_{1}-\theta_{2}\right\|_{L^{p}(\Omega)}^{p} d s+M_{2} \int_{0}^{t}\left\|c_{1}-c_{2}\right\|_{L^{p}(\Omega)}^{p} d s(2.3 .2)
$$

where $p_{i}, m_{i}$ is the solution corresponding to $\left(\theta_{i}, c_{i}\right)$, and $|\cdot|$ is the Euclidean norm in $\mathbb{R}^{2}$.

We will derive global existence on the interval $(0, \mathrm{~T})$ making use of the following classical result, which can be found for instance in [11, Theorem 1.1, Chap. 2]

Theorem 2.3.1 (Theorem of Carathéodory). We consider the initial value problem:

$$
\left\{\begin{array}{l}
u^{\prime}(t)=F(u(t), t) \quad \text { on } J \\
u\left(t_{0}\right)=u_{0}
\end{array}\right.
$$

where $J \subset \mathbb{R}$ with $t_{0} \in J$ and the map $F$ is of the form $F: K \times J \rightarrow \mathbb{R}^{N}$, where $K$ is a compact set in $\mathbb{R}^{N}$. We assume:

(i) $F: K \times J \rightarrow \mathbb{R}^{N}$ satisfies the Carathéodory condition, i.e.

- $t \rightarrow F_{i}(u, t)$ is measurable on $J$ for each $u \in K$

- $u \rightarrow F_{i}(u, t)$ is continuous on $K$ for almost all $t \in J$

(ii) There exists a Lebesgue-integrable function $M: J \rightarrow \mathbb{R}$ such that $\left|F_{i}(t, u)\right|<M(t)$ for all $(t, u) \in J \times K$ and all $i$.

Then there exists a solution $u$ of problem $(P)$ defined on a neighbourhood $U$ of $t_{0}$, 'in the extended sense', which means that $u$ is absolutely continuous, $u(\cdot)$ satisfies $(P)$ almost everywhere on $U$ and satisfies the initial condition.

Now we can start the proof of Lemma 2.3.1. 
Proof of Lemma 2.3.1 In order to prove (a) it is convenient to rewrite problem (2.3.1) as:

$$
\begin{aligned}
z_{t} & =F(z, t) \quad \text { in }(0, T) \\
z(0) & =0
\end{aligned}
$$

with $F(z, \cdot)=f(z, \theta(\cdot), c(\cdot))$.

First of all we are going to show that the hypothesis $(i),(i i)$ of the Carathéodory's Theorem are satisfied, with $K=[0,1] \times[0,1]$ and $J=(0, T)$ :

$(i)$

- $t \rightarrow F(z, t)$ is measurable on $(0, T)$ for each $z \in[0,1] \times[0,1]$;

- $z \rightarrow F(z, t)$ is continuous on $[0,1] \times[0,1]$ for almost all $t \in(0, T)$.

These conditions follow from the definition of $F$ as a consequence of the measurability of $\theta$ and $c$ on $(0, T)$ and of the fact that $g_{1}(\theta, c), g_{2}(\theta, c)$ are Lipschitz continuous in both variables.

(ii) Using assumption (A4),(A5) we have

$$
\begin{gathered}
\left|F_{1}(z, t)\right| \leq|1-p-m| g_{1}(\theta, c) \leq \gamma_{1} \quad \text { on } \quad[0,1] \times[0,1] \times(0, T), \\
\left|F_{2}(z, t)\right| \leq|\bar{m}-m| g_{2}(\theta, c) \leq \gamma_{2} \quad \text { on } \quad[0,1] \times[0,1] \times(0, T) .
\end{gathered}
$$

According to Carathéodory Theorem, system (2.3.3) has a solution in the extended sense on some time interval $\left[0, T_{+}\right)$, for some $T_{+}>0$.

Next we are going to show that the solution is globally unique. To this end it is sufficient to prove that there holds

$$
\left|F\left(z_{1}, t\right)-F\left(z_{2}, t\right)\right| \leq L\left|z_{1}-z_{2}\right| \quad \forall\left(z_{1}, t\right),\left(z_{2}, t\right) \in[0,1] \times[0,1] \times(0, T) .
$$

Indeed, according to the definition of $F$ :

$$
\begin{aligned}
& \left|F\left(z_{1}, t\right)-F\left(z_{2}, t\right)\right|^{2}=\left|\left(1-p_{1}-m_{1}\right) g_{1}(t)-\left(1-p_{2}-m_{2}\right) g_{1}(t)\right| \\
& \quad+\left|\left[\min \left\{\bar{m}(t) ; 1-p_{1}\right\}-m_{1}\right]_{+} g_{2}(t)-\left[\min \left\{\bar{m}(t) ; 1-p_{2}\right\}-m_{2}\right]_{+} g_{2}(t)\right| .
\end{aligned}
$$

Thanks to the boundedness of $g_{1}$ and $g_{2}$, we obtain $\left|\left(1-p_{1}-m_{1}\right) g_{1}(t)-\left(1-p_{2}-m_{2}\right) g_{1}(t)\right| \leq \gamma_{1}\left(\left|p_{1}-p_{2}\right|+\left|m_{2}-m_{1}\right|\right)$ and

$$
\begin{array}{r}
\left|\left[\min \left\{\bar{m}(t) ; 1-p_{1}\right\}-m_{1}\right]_{+} g_{2}(t)-\left[\min \left\{\bar{m}(t) ; 1-p_{2}\right\}-m_{2}\right]_{+} g_{2}(t)\right| \\
\leq \gamma_{2}\left|\min \left\{\bar{m}(t) ; 1-p_{1}\right\}-m_{1}-\min \left\{\bar{m}(t) ; 1-p_{2}\right\}+m_{2}\right| .
\end{array}
$$

We shall now distinguish some cases.

If either $\min \left\{\bar{m}(t) ; 1-p_{i}\right\}=1-p_{i}$ or $\min \left\{\bar{m}(t) ; 1-p_{i}\right\}=\bar{m}(t)$, for $i=1,2,(2.3 .4)$ immediately follows. 
If $\min \left\{\bar{m}(t) ; 1-p_{1}\right\}=1-p_{1}$ and $\min \left\{\bar{m}(t) ; 1-p_{2}\right\}=\bar{m}(t)$ (the same holds for inverted indices), we have $p_{2}<1-\bar{m}<p_{1}$, therefore

$$
\begin{aligned}
& \gamma_{2}\left|\min \left\{\bar{m}(t) ; 1-p_{1}\right\}-m_{1}-\min \left\{\bar{m}(t) ; 1-p_{2}\right\}+m_{2}\right| \\
& \quad \leq \gamma_{2}\left(\left|m_{1}-m_{2}\right|+\left|1-p_{1}-\bar{m}(t)\right|\right) \leq \gamma_{2}\left(\left|m_{1}-m_{2}\right|+\left|p_{1}-p_{2}\right|\right) .
\end{aligned}
$$

Thus, there exists a positive constant $L$ such that

$$
\left|F\left(z_{1}, t\right)-F\left(z_{2}, t\right)\right| \leq L\left|z_{1}-z_{2}\right| \quad \forall\left(z_{1}, t\right),\left(z_{2}, t\right) \in[0,1] \times[0,1] \times(0, T) .
$$

Hence we have proved uniqueness of $z$ on $(0, T)$.

To ensure global existence of the solution we need more an a priori estimate on an arbitrary subinterval of $(0, T)$ (see for instance [52], pp. 799-800). To this aim we introduce the variable $Z=p+m$ and define $T_{\epsilon}$ as the maximal time such that $Z<1-\epsilon$ on $\left(0, T_{\epsilon}\right)$.

We will show that for any $T>0$ there exists an $\epsilon$ such that $0 \leq Z \leq 1-\epsilon$ in $(0, T)$. By this means, we will have also shown the last statement of part (a) of the lemma. This will be done by means of a classical comparison criterium for ODEs (see for instance [28, Prop. 3.1, Chap. 1]). $Z$ satisfies, on $\left[0, T_{\epsilon}\right)$ :

$$
\begin{aligned}
\dot{Z}(t) & =(1-Z(t)) g_{1}(t)+[\min \{\bar{m}(t) ; 1-p(t)\}-m(t)]_{+} g_{2}(t) \\
& \leq g(t, Z(t)):=(1-Z(t))\left(g_{1}(t)+g_{2}(t)\right), \\
Z(0) & =0 .
\end{aligned}
$$

Now, if we consider on $[0, T]$ the auxiliary problem:

$$
\begin{aligned}
\dot{V}(t) & =(1-V(t))\left(g_{1}(t)+g_{2}(t)\right)=g(t, V(t)) \\
V(0) & =0
\end{aligned}
$$

the solution is given by

$$
V(t)=1-e^{-\int_{0}^{t}\left(g_{1}+g_{2}\right)(s) d s} \quad \forall t \in[0, T]
$$

and we immediately have that there exists a constant $c_{T}>0$ such that:

$$
0 \leq V(t) \leq c_{T}<1 \quad \text { on } \quad[0, T]
$$

Notice that $g(t, V(t))=(1-V(t))\left(g_{1}(t)+g_{2}(t)\right)$ is Lipschitz continuous on $[0, T]$ with respect to $V$. Thus, choosing $\epsilon=1-c_{T}$, we have

$$
Z(t) \leq V(t) \leq 1-\epsilon \quad \text { on } \quad[0, T]
$$

Since $T_{\epsilon}$ was chosen maximally such that $Z(t) \leq 1-\epsilon$ on $\left(0, T_{\epsilon}\right)$, it follows that $T_{\epsilon} \geq T$.

Now, since $Z<1-\epsilon$, we have, by definition, that $p_{t}$ and $m_{t}$ are positive with $p(0)=$ $m(0)=0$. Therefore $p \geq 0$ and $m \geq 0$ on $(0, T)$. It follows that

$$
0 \leq p+m \leq c_{T}<1 \text { on }(0, T)
$$


Thus part $(a)$ of Lemma 2.3.1 is concluded.

(b) Let us consider again the equation $z_{t}=f(z, \theta, c)$. Let $z_{i}$ be the solution to the system (2.3.1), corresponding to $\theta_{i}, c_{i}, i=1,2$. Denoting $z=z_{1}-z_{2}$, subtracting the equations and taking the scalar product with the function $|z|^{p-2} z$, we obtain:

$$
\frac{1}{p} \int_{\Omega}|z(t)|^{p} d x=\int_{0}^{t} \int_{\Omega}\left(f\left(z_{1}, \theta_{1}, c_{1}\right)-f\left(z_{1}, \theta_{2}, c_{2}\right)\right) \cdot z|z|^{p-2} d x d s .
$$

Invoking (A4), $f$ is Lipschitz-continuous in all variables, thus, proceeding from (2.3.6), the conclusion follows through standard application of Young's inequality and Gronwall lemma. The proof of Lemma 2.3.1 is thus completed.

Next, we define

$$
B(\theta, c):=\rho L_{p}(\theta) p_{t}+\rho L_{m}(\theta) m_{t},
$$

where $(p, m)$ depends on $\theta, c$ as characterized by the previous lemma.

Lemma 2.3.2. Suppose that (A2), (A4), (A5) hold. Then the operator $B$ defined by (2.3.7) has the following properties

(a) There exists a constant $\bar{B}$ independent of $\theta$, c such that, for all $\theta \in L^{2}\left(Q_{T}\right)$, $c \in L^{2}\left(Q_{T}\right)$ there holds

$$
\|B(\theta, c)\|_{L^{\infty}\left(Q_{T}\right)} \leq \bar{B}
$$

(b) Given $c \in L^{2}\left(Q_{T}\right)$, let $\theta_{k} \subset L^{2}\left(Q_{T}\right)$ be any sequence converging strongly in $L^{2}\left(Q_{T}\right)$ to $\theta \in L^{2}\left(Q_{T}\right)$. Then for every $p \in[1, \infty)$, we have

$$
B\left(\theta_{k}, c\right) \rightarrow B(\theta, c) \quad \text { strongly in } L^{p}\left(Q_{T}\right)
$$

(c) There are constants $K_{1}, K_{2}>0$ such that for all $\theta_{1}, \theta_{2}, c_{1}, c_{2} \in L^{2}\left(Q_{T}\right)$ and for almost all $x \in \Omega$ and every $t \in(0, T)$

$$
\begin{aligned}
& \int_{0}^{t}\left|B\left(\theta_{1}(x, s), c_{1}(x, s)\right)-B\left(\theta_{2}(x, s), c_{2}(x, s)\right)\right|^{2} d s \\
& \leq K_{1} \int_{0}^{t}\left|\theta_{1}(x, s)-\theta_{2}(x, s)\right|^{2} d s+K_{2} \int_{0}^{t}\left|c_{1}(x, s)-c_{2}(x, s)\right|^{2} d s .
\end{aligned}
$$

Proof of Lemma 2.3.2

(a) follows directly from assumptions (A2),(A4),(A5) and Lemma 2.3.1 (a). 
(b) Emphasizing the dependence of $p$ and $m$ from the $c$ and $\theta$, as possible according to the previous lemma, we have

$$
\begin{aligned}
\frac{\partial p_{\theta, c}}{\partial t} & =(1-p-m) g_{1}(\theta, c) \\
\frac{\partial m_{\theta, c}}{\partial t} & =[\min \{\bar{m}(\theta, c) ; 1-p\}-m]_{+} g_{2}(\theta, c) .
\end{aligned}
$$

Let $x \in \Omega \backslash N$, with $N \subset \Omega$ of zero measure and consider $z=(p, m)^{T}$. By Lemma 2.3.1 $(a),\left\|z_{\theta_{k}}\right\|_{W^{1, \infty}\left(0, T ; L^{\infty}(\Omega)\right)} \leq M \forall k$, thus $\left\|z_{\theta_{k}}\right\|_{W^{1, p}\left(0, T ; L^{\infty}(\Omega)\right)} \leq M \forall k, \forall p<\infty$. Thus, there exists a subsequence, $\left\{\theta_{k^{\prime}}\right\}$, and some $\hat{z}$ such that

$$
z_{\theta_{k^{\prime}}}(x, \cdot) \rightarrow \hat{z}(x, \cdot) \quad \text { weakly }- \text { star } \quad \text { in } \quad W^{1, \infty}(0, T), \quad \text { for a.e. } x \in \Omega .
$$

Thus, we have

$$
\begin{aligned}
& \frac{\partial z_{\theta_{k^{\prime}}}}{\partial t}(x, \cdot) \rightarrow \frac{\partial \hat{z}}{\partial t}(x, \cdot) \quad \text { weakly } \quad \text { in } L^{p}(0, T) \quad \forall p<\infty \text {, for a.e. } x \in \Omega \text {. } \\
& z_{\theta_{k}^{\prime}}(x, \cdot) \rightarrow \hat{z}(x, \cdot) \quad \text { strongly } \quad \text { in } \quad C[0, T], \quad \text { for a.e. } x \in \Omega .
\end{aligned}
$$

Since the solution to system (2.3.1) is unique, we have $\hat{z}(x, \cdot)=z_{\theta}(x, \cdot)$ and the convergence holds for the whole sequence, hence we can conclude that $z_{\theta_{k}}(x, t) \rightarrow$ $z_{\theta}(x, t)$ pointwise in $Q_{T}$. Since $\theta_{k} \rightarrow \theta$ strongly in $L^{2}\left(Q_{T}\right)$, using assumption (A4), possibly extracting a subsequence, we have

$$
\rho L_{p}\left(\theta_{k^{\prime}}\right) \frac{\partial p_{\theta_{k^{\prime}}}}{\partial t}+\rho L_{m}\left(\theta_{k^{\prime}}\right) \frac{\partial m_{\theta_{k^{\prime}}}}{\partial t} \rightarrow \rho L_{p}(\theta) \frac{\partial p_{\theta}}{\partial t}+\rho L_{m}(\theta) \frac{\partial m_{\theta}}{\partial t} \quad \text { a.e } \quad \text { in } Q_{T} .
$$

But, applying Lebesgue theorem, we get

$$
B\left(\theta_{k^{\prime}}, c\right) \rightarrow B(\theta, c) \quad \text { strongly in } L^{p}\left(Q_{T}\right) .
$$

Since the limit does not depend on the extracted subsequence the convergence holds for the whole sequence $\left\{\theta_{k}\right\}$, hence we obtain (2.3.8).

(c) follows directly from assumption (A2) and Lemma 2.3.1 (b).

Lemma 2.3.3. Suppose (A2),(A4) hold. Let $\hat{c} \in L^{2}\left(0, T ; L^{2}(\Omega)\right)$. Then there exists a unique $\theta(\hat{c}) \in W_{2}^{2,1}\left(Q_{T}\right)$ and a unique $z(\hat{c})=(p(\hat{c}), m(\hat{c})) \in W^{1, \infty}\left(0, T ; L^{\infty}(\Omega)\right) \times$ $W^{1, \infty}\left(0, T ; L^{\infty}(\Omega)\right)$, satisfying

$$
\begin{aligned}
\rho \alpha(\theta) \frac{\partial \theta}{\partial t}-k \Delta \theta=B(\theta, \hat{c}) & \text { in } Q_{T} \\
-k \frac{\partial \theta}{\partial \nu}=h\left(\theta-\theta_{\Gamma}\right) & \text { on } \partial \Omega \times(0, T) \\
\theta(x, 0)=\theta_{0} & \text { in } \Omega \\
z_{t}=f(z, \theta, \hat{c}) & \text { in } Q_{T} \\
z(0)=0 & \text { in } \Omega .
\end{aligned}
$$

where $f$ is defined as in (2.3.1a). Moreover, there exist $\lambda_{1}, \lambda_{2}>0$ such that

$$
\left\|\theta_{1}-\theta_{2}\right\|_{L^{2}\left(0, t ; L^{2}(\Omega)\right)}^{2} \leq \lambda_{1} \int_{0}^{t}\left\|\hat{c}_{1}-\hat{c}_{2}\right\|_{L^{2}\left(0, s ; L^{2}(\Omega)\right)}^{2} d s
$$


and

$$
\left\|\left|z_{1}-z_{2}\right|\right\|_{L^{2}\left(0, t ; L^{2}(\Omega)\right)}^{2} \leq \lambda_{2} \int_{0}^{t}\left\|\hat{c}_{1}-\hat{c}_{2}\right\|_{L^{2}\left(0, s ; L^{2}(\Omega)\right)}^{2} d s,
$$

where $\left(\theta_{i}, z_{i}\right)$ is the solution corresponding to $\hat{c}_{i}, i=1,2$.

Proof of Lemma 2.3.3 Existence. We introduce the operator

$$
\begin{gathered}
P: L^{2}\left(Q_{T}\right) \rightarrow L^{2}\left(Q_{T}\right), \\
\theta=P \hat{\theta},
\end{gathered}
$$

by demanding $\theta$ to be the solution of the linear parabolic problem

$$
\begin{aligned}
\rho \alpha(\hat{\theta}) \frac{\partial \theta}{\partial t}-k \triangle \theta=B(\hat{\theta}, \hat{c}) & \text { in } Q_{T} \\
-k \frac{\partial \theta}{\partial \nu}=h\left(\theta-\theta_{\Gamma}\right) & \text { on } \partial \Omega \times(0, T) \\
\theta(x, 0)=\theta_{0} & \text { in } \Omega .
\end{aligned}
$$

According to classical results about parabolic equations, problem (2.3.13) has a unique strong solution $\theta \in W_{2}^{1,1}\left(Q_{T}\right)$ (see [33][Chap. 3, Theorem 6.1- Remark 6.2]), therefore the operator $P$ is well-defined. Further, applying classical regularity results for elliptic equations it yields $\theta \in W_{2}^{2,1}\left(Q_{T}\right)$. Moreover, thanks to Lemma 2.3.2 (a), there exists a constant $M>0$, independent of $\hat{\theta}$, such that:

$$
\|\theta\|_{W_{2}^{2,1}\left(Q_{T}\right)} \leq M
$$

We shall now show the continuity of the operator $P$.

Let $\left(\hat{\theta}_{n}\right) \subset L^{2}\left(Q_{T}\right)$ with $\hat{\theta}_{n} \rightarrow \hat{\theta}$ strongly in $L^{2}\left(Q_{T}\right)$. Defining $\theta_{n}=P \hat{\theta}_{n}$, in view of (2.3.14), $\left\|\theta_{n}\right\|_{W_{2}^{2,1}\left(Q_{T}\right)} \leq M$. Thus, we can find a sub-sequence $\hat{\theta}_{n^{\prime}}$ such that

$$
\begin{array}{ll}
\theta_{n^{\prime}} \rightarrow \theta & \text { weakly in } \quad W_{2}^{2,1}\left(Q_{T}\right), \quad \text { strongly in } L^{2}\left(Q_{T}\right), \\
\theta_{n^{\prime}} \rightarrow \theta & \text { a.e. in } Q_{T} .
\end{array}
$$

Testing equation (2.3.13a), written for the index $n^{\prime}$, by $\phi \in L^{2}\left(0, t ; W_{2}^{1}(\Omega)\right)$, we get

$$
\begin{aligned}
& \int_{0}^{t} \int_{\Omega} \rho \alpha\left(\hat{\theta}_{n^{\prime}}\right) \frac{\partial \theta_{n^{\prime}}}{\partial s} \phi d x d s+k \int_{0}^{t} \int_{\Omega} \nabla \theta_{n^{\prime}} \nabla \phi d x d s \\
& +\int_{0}^{t} \int_{\partial \Omega} h(\sigma)\left(\theta_{n^{\prime}}-\theta_{\Gamma}\right) \phi d \sigma d s-\int_{0}^{t} \int_{\Omega} B\left(\hat{\theta}_{n^{\prime}}, \hat{c}\right) \phi d x d s=0 .
\end{aligned}
$$

By means of $(2.3 .15 \mathrm{a}),(2.3 .15 \mathrm{~b})$ we can pass to the limit in the last three terms of (2.3.16). We can break the first term in two terms

$\rho \int_{0}^{t} \int_{\Omega} \alpha\left(\hat{\theta}_{n^{\prime}}\right) \frac{\partial \theta_{n^{\prime}}}{\partial s} \phi d x d s=\rho \int_{0}^{t} \int_{\Omega} \alpha\left(\hat{\theta}_{n^{\prime}}\right)\left(\frac{\partial \theta_{n^{\prime}}}{\partial s}-\frac{\partial \theta}{\partial s}\right) \phi d x d s+\rho \int_{0}^{t} \int_{\Omega} \alpha\left(\hat{\theta}_{n^{\prime}}\right) \frac{\partial \theta}{\partial s} \phi d x d s$. 
Thanks to the continuity of $\alpha$, we have that

$$
\alpha\left(\hat{\theta}_{n^{\prime}}\right) \phi \rightarrow \alpha(\hat{\theta}) \phi \quad \text { a.e. in } Q_{T}
$$

thus, using Lebesgue theorem, $\rho \alpha\left(\hat{\theta}_{n^{\prime}}\right) \phi \rightarrow \rho \alpha(\hat{\theta}) \phi$ strongly in $L^{2}\left(Q_{T}\right)$ while $\frac{\partial \theta_{n^{\prime}}}{\partial s} \rightarrow \frac{\partial \theta}{\partial s}$ weakly in $L^{2}\left(Q_{T}\right)$. Thus, $\int_{0}^{t} \int_{\Omega} \alpha\left(\hat{\theta}_{n^{\prime}}\right)\left(\frac{\partial \theta_{n^{\prime}}}{\partial s}-\frac{\partial \theta}{\partial s}\right) \phi d x d s \rightarrow 0$ and

$$
\rho \int_{0}^{t} \int_{\Omega} \alpha\left(\hat{\theta}_{n^{\prime}}\right) \frac{\partial \theta_{n^{\prime}}}{\partial s} \phi d x d s \rightarrow \rho \int_{0}^{t} \int_{\Omega} \alpha(\hat{\theta}) \frac{\partial \theta}{\partial s} \phi d x d s
$$

Hence we have obtained

$$
\begin{aligned}
& \rho \int_{0}^{t} \int_{\Omega} \alpha(\hat{\theta}) \theta_{s} \phi d x d s+k \int_{0}^{t} \int_{\Omega} \nabla \theta \nabla \phi d x d s \\
& +\int_{0}^{t} \int_{\partial \Omega} h(\sigma)\left(\theta-\theta_{\Gamma}\right) \phi d \sigma d s-\int_{0}^{t} \int_{\Omega} B(\hat{\theta}, \hat{c}) \phi d x d s=0 .
\end{aligned}
$$

As the solution to the parabolic system (2.3.13) is unique, we have

$$
\theta=P \hat{\theta} \quad \text { a.e. in } Q_{T}
$$

and, since the limit does not depend on the extracted sub-sequence, it follows that

$$
P \hat{\theta}_{n} \rightarrow P \hat{\theta}
$$

weakly in $W_{2}^{2,1}\left(Q_{T}\right)$ and strongly in $L^{2}\left(Q_{T}\right)$.

Now, let

$$
K:=\left\{u \in L^{2}\left(Q_{T}\right):\|u\|_{W_{2}^{2,1}\left(Q_{T}\right)} \leq M\right\} .
$$

$K$ is non-empty, convex, closed and relatively compact subset of $L^{2}\left(Q_{T}\right)$ and $P: K \subset$ $L^{2}\left(Q_{T}\right) \rightarrow K$ is a continuous mapping. By the Schauder fixed point theorem, there exists a fixed point of the mapping $P$, i.e. there exists a weak solution $\theta \in W_{2}^{2,1}\left(Q_{T}\right)$ to $(2.3 .10 \mathrm{a}),(2.3 .10 \mathrm{~b}),(2.3 .10 \mathrm{c})$. Since the equation $z_{t}=f(z, \theta, \hat{c})$ with initial condition has already been solved and incorporated in the right-hand side $B(\theta, \hat{c})$, the proof provides actually the existence of a solution for the whole system (2.3.10a)-(2.3.10e). Now we must prove (2.3.11), which can be seen as a kind of stability of the solution $\theta$ with respect to given $\theta$ and $c$.

Uniqueness and stability. Let

$$
J(\theta):=\int_{0}^{\theta} \rho \alpha(\xi) d \xi
$$


Integration of (1.4.6) with respect to time leads to

$$
\int_{0}^{t} B(\theta, c)(x, s) d s=J(\theta(x, t))-J\left(\theta_{0}(x)\right)-k \Delta \int_{0}^{t} \theta(x, s) d s .
$$

Now, let $\theta_{1}, \theta_{2} \in W_{2}^{2,1}\left(Q_{T}\right)$ be solutions to the system (2.3.13) corresponding to $\hat{c}_{1}, \hat{c}_{2}$ respectively. Inserting these solutions into (2.3.18), subtracting both equations, and testing by $\theta:=\theta_{1}-\theta_{2}$, we find

$$
\begin{gathered}
\int_{0}^{t} \int_{\Omega}\left(\int_{0}^{s} B\left(\theta_{1}(x, \xi), \hat{c}_{1}(x, \xi)\right)-B\left(\theta_{2}(x, \xi), \hat{c}_{2}(x, \xi)\right) d \xi\right) \theta(x, s) d x d s \\
=\int_{0}^{t} \int_{\Omega}\left[J\left(\theta_{1}(x, s)\right)-J\left(\theta_{2}(x, s)\right)\right] \theta(x, s) d x d s+k \int_{0}^{t} \int_{\Omega} \nabla\left(\int_{0}^{s} \theta(x, \xi) d \xi\right) \nabla \theta(x, s) d x d s \\
+\int_{0}^{t} \int_{\partial \Omega}\left(\int_{0}^{s} h(\sigma) \theta(\sigma, \xi) d \xi\right) \theta(\sigma, s) d \sigma d s .
\end{gathered}
$$

Concerning the last term we can see that

$$
\begin{aligned}
& \int_{0}^{t} \int_{\partial \Omega}\left(\int_{0}^{s} h(\sigma) \theta(\sigma, \xi) d \xi\right) \theta(\sigma, s) d \sigma d s=\int_{0}^{t} \int_{\partial \Omega} h(\sigma)\left(\int_{0}^{s} \theta(\sigma, \xi) d \xi\right) \theta(\sigma, s) d \sigma d s \\
& =\frac{1}{2} \int_{0}^{t} \int_{\partial \Omega} h(\sigma) \frac{d}{d s}\left(\int_{0}^{s} \theta(\sigma, \xi) d \xi\right)^{2} d \sigma d s=\frac{1}{2} \int_{\partial \Omega} h(\sigma)\left(\int_{0}^{t} \theta(\sigma, s) d s\right)^{2} d \sigma .
\end{aligned}
$$

Thus, from (2.3.19) we obtain

$$
\begin{aligned}
& \left.\int_{0}^{t} \int_{\Omega}\left(\int_{0}^{s} B\left(\theta_{1}(x, \xi), \hat{c}_{1}(x, \xi)\right)-B\left(\theta_{2}(x, \xi), \hat{c}_{2}(x, \xi)\right)(x, \xi)\right) d \xi\right) \theta(x, s) d x d s \\
& \geq \alpha_{0} \rho \int_{0}^{t} \int_{\Omega} \theta^{2}(x, s) d x d s+\frac{k}{2} \int_{\Omega}\left|\nabla \int_{0}^{t} \theta(x, s) d s\right|^{2} d x \\
& +\frac{1}{2} \int_{\partial \Omega} h(\sigma)\left(\int_{0}^{t} \theta(\sigma, s) d s\right)^{2} d \sigma \geq \alpha_{0} \rho \int_{0}^{t} \int_{\Omega} \theta^{2}(x, s) d x d s .
\end{aligned}
$$


Using Holder's and Young's inequalities and Lemma 2.3.1 it follows that

$$
\begin{aligned}
& \left|\int_{0}^{t} \int_{\Omega}\left(\int_{0}^{s} B\left(\theta_{1}(x, \xi), \hat{c}_{1}(x, \xi)\right)-B\left(\theta_{2}(x, \xi), \hat{c}_{2}(x, \xi)\right) d \xi\right) \theta(x, s) d x d s\right| \\
& \leq \frac{1}{4 \delta} \int_{0}^{t} \int_{\Omega}\left(\int_{0}^{s} B\left(\theta_{1}(x, \xi), \hat{c}_{1}(x, \xi)\right)-B\left(\theta_{2}(x, \xi), \hat{c}_{2}(x, \xi)\right) d \xi\right)^{2} d x d s \\
& +\delta \int_{0}^{t}\|\theta(\cdot, s)\|_{L^{2}(\Omega)}^{2} d s \\
& \leq \frac{T}{4 \delta} \int_{0}^{t} \int_{\Omega} \int_{0}^{s}\left(K_{1}\left|\theta_{1}(x, \xi)-\theta_{2}(x, \xi)\right|^{2}+K_{2}\left|\hat{c}_{1}(x, \xi)-\hat{c}_{2}(x, \xi)\right|^{2}\right) d \xi d x d s \\
& +\delta \int_{0}^{t}\|\theta(\cdot, s)\|_{L^{2}(\Omega)}^{2} d s \\
& \leq \frac{C T}{4 \delta} \int_{0}^{t}\|\theta\|_{L^{2}\left(0, s ; L^{2}(\Omega)\right)}^{2} d s+\frac{C T}{4 \delta} \int_{0}^{t}\|\hat{c}\|_{L^{2}\left(0, s ; L^{2}(\Omega)\right)}^{2} d s+\delta \int_{0}^{t}\|\theta(\cdot, s)\|_{L^{2}(\Omega)}^{2} d s,
\end{aligned}
$$

where $C=\min \left\{\mathrm{K}_{1}, \mathrm{~K}_{2}\right\}$. Thus, we have

$$
\begin{aligned}
\alpha_{0} \rho \int_{0}^{t} \int_{\Omega} \theta^{2}(x, s) d x d s & \leq \frac{C T}{4 \delta} \int_{0}^{t}\|\theta\|_{L^{2}\left(0, s ; L^{2}(\Omega)\right)}^{2} d s \\
& +\frac{C T}{4 \delta} \int_{0}^{t}\|\hat{c}\|_{L^{2}\left(0, s ; L^{2}(\Omega)\right)}^{2} d s+\delta \int_{0}^{t}\|\theta(\cdot, s)\|_{L^{2}(\Omega)}^{2} d s .
\end{aligned}
$$

Choosing $\delta=\frac{\alpha_{0} \rho}{2}$ we have:

$$
\|\theta\|_{L^{2}\left(0, t ; L^{2}(\Omega)\right)}^{2} \leq M_{1} \int_{0}^{t}\|\theta\|_{L^{2}\left(0, s ; L^{2}(\Omega)\right)}^{2} d s+M_{2} \int_{0}^{t}\|\hat{c}\|_{L^{2}\left(0, s ; L^{2}(\Omega)\right)}^{2} d s
$$

with constants $M_{1}, M_{2}>0$.

Hence, applying Gronwall lemma, we find a constant $C_{1}$ such that

$$
\left\|\theta_{1}-\theta_{2}\right\|_{L^{2}\left(0, t ; L^{2}(\Omega)\right)}^{2} \leq C_{1} \int_{0}^{t}\left\|\hat{c}_{1}(s)-\hat{c}_{2}(s)\right\|_{L^{2}\left(0, s ; L^{2}(\Omega)\right)}^{2} d s .
$$

Therefore (2.3.11) is proved. Inequality (2.3.12) follows immediately from Lemma 2.3.1 $(b)$ and estimate (2.3.11). The proof of Lemma 2.3.3 is thus completed. 
Now, we are in a position to prove Theorem 2.2.1.

Proof of Theorem 2.2.1

Let us denote

$$
\mu(\theta, c):=(1-p-m) D(\theta, c) .
$$

We note that, in view of (A6) and Lemma 2.3.1 (b), $\mu$ is Lipschitz-continuous with respect to $\theta$ and $c$.

We define an operator

$$
\begin{gathered}
\mathcal{T}: L^{2}\left(Q_{T}\right) \longrightarrow L^{2}\left(Q_{T}\right), \\
\mathcal{T} \hat{c}=c,
\end{gathered}
$$

by demanding $c$ to be the solution of the parabolic problem

$$
\begin{aligned}
\frac{\partial c}{\partial t}-\operatorname{div}\left(\mu_{\hat{c}} \nabla c\right) & =0 & & \text { in } Q_{T} \\
-\mu_{\hat{c}} \frac{\partial c}{\partial \nu} & =\beta\left(c-c_{p}\right) & & \text { on } \partial \Omega \times(0, T) \\
c(x, 0) & =c_{0} & & \text { in } \Omega .
\end{aligned}
$$

where $\mu_{\hat{c}}=\left(1-p_{\hat{c}}-m_{\hat{c}}\right) D\left(\theta_{\hat{c}}, \hat{c}\right),\left(\theta_{\hat{c}}, p_{\hat{c}}, m_{\hat{c}}\right)$ being the solution to problem (2.3.10), with respect to given $\hat{c}$. Denoting

$$
\begin{aligned}
a(c, \phi ; t) & :=\int_{\Omega} \mu_{\hat{c}} \nabla c \nabla \phi d x+\int_{\partial \Omega} \beta c \phi d \sigma, \\
\langle f(t), \phi\rangle & :=\int_{\partial \Omega} \beta c_{p} \phi d \sigma, \quad \phi \in W_{2}^{1}(\Omega)
\end{aligned}
$$

we have that problem (2.3.22) is equivalent to the following one. We seek a function $c$ such that, for all $\phi \in W_{2}^{1}(\Omega)$ and a.e in $t \in(0, T)$

$$
\begin{aligned}
\left\langle\frac{d}{d t} c(t), \phi\right\rangle+a(c(t), \phi ; t) & =\langle f(t), \phi\rangle, \\
c(0) & =c_{0} \\
c & \in W(0, T),
\end{aligned}
$$

where $\langle$,$\rangle denotes the duality between W_{2}^{1}(\Omega)$ and $\left(W_{2}^{1}(\Omega)\right)^{*}$.

In view of (A3),(A6),(A7), problem (2.3.23) admits a unique solution $c \in W(0, T)$ (cf. [36, Theorem 1.2, Chap. 2]). Moreover, there exists a constant $M$ independent of $\hat{c}$, such that:

$$
\|c\|_{W(0, T)} \leq M
$$


To derive the continuity of the operator $\mathcal{T}$, let $\left\{\hat{c}_{n}\right\} \subset L^{2}\left(0, T ; L^{2}(\Omega)\right)$, with $\hat{c}_{n} \rightarrow \hat{c}$ strongly in $L^{2}\left(Q_{T}\right)$. Defining $c_{n}=\mathcal{T} \hat{c}_{n}$, thanks to $(2.3 .24)$, we have $\left\|c_{n}\right\|_{W(0, T)} \leq M$. Thus, there exists a sub-sequence $\left\{\hat{c}_{n^{\prime}}\right\}$ such that

$$
c_{n^{\prime}} \longrightarrow c \text { weakly in } W(0, T) \text {. }
$$

We test $(2.3 .22 \mathrm{a})$ by

$$
\Phi(x, t)=\psi(t) \phi(x) \quad \text { with } \quad \psi \in C^{1}[0, T], \psi(T)=0, \phi \in W_{2}^{1}(\Omega) .
$$

Denoting $\mathcal{T} \hat{c}_{n^{\prime}}:=c_{n^{\prime}}$, we have

$$
\int_{0}^{T} \int_{\Omega} \frac{\partial c_{n^{\prime}}}{\partial s} \Phi d x d s+\int_{0}^{T} \int_{\Omega} \mu_{\hat{c}_{n^{\prime}}} \nabla c_{n^{\prime}} \nabla \Phi d x d s+\int_{0}^{T} \int_{\partial \Omega} \beta\left(c_{n^{\prime}}-c_{p}\right) \Phi d \sigma d s=0 .
$$

Concerning the first term in (2.3.27) we have

$$
\int_{0}^{T} \int_{\Omega} \frac{\partial c_{n^{\prime}}}{\partial s} \Phi d x d s=-\int_{\Omega} c_{n^{\prime}}(x, 0) \Phi(x, 0) d x-\int_{0}^{T} \int_{\Omega} c_{n^{\prime}} \frac{\partial \Phi}{\partial s} d x d s .
$$

Now,

$$
\int_{\Omega} c_{n^{\prime}}(x, 0) \Phi(x, 0) d x=\int_{\Omega} c_{0} \Phi(x, 0) d x,
$$

and, by virtue of (2.3.25),

$$
\int_{0}^{T} \int_{\Omega} c_{n^{\prime}} \frac{\partial \Phi}{\partial s} d x d s \rightarrow \int_{0}^{T} \int_{\Omega} c \frac{\partial \Phi}{\partial s} d x d s
$$

The second term can be rearranged as

$$
\int_{0}^{T} \int_{\Omega} \mu_{\hat{c}_{n^{\prime}}} \nabla c_{n^{\prime}} \nabla \Phi d x d s=\int_{0}^{T} \int_{\Omega} \mu_{\hat{c}_{n^{\prime}}}\left(\nabla c_{n^{\prime}}-\nabla c\right) \nabla \Phi d x d s+\int_{0}^{T} \int_{\Omega} \mu_{\hat{c}_{n^{\prime}}} \nabla c \nabla \Phi d x d s .
$$

Since $\mu$ is continuous and bounded as a function of $c$, possibly extracting a subsequence, we obtain: $\mu_{\hat{c}_{n^{\prime}}}(x, t) \rightarrow \mu_{\hat{c}}(x, t)$ a.e in $Q_{T}$, therefore $\mu_{\hat{c}_{n^{\prime}}}(x, t) \nabla \Phi \rightarrow \mu_{\hat{c}}(x, t) \nabla \Phi$ pointwise, moreover $\mu_{\hat{c}_{n^{\prime}}}(x, t) \nabla \Phi$ is bounded in $L^{2}$ thus, using Lebesgue theorem, we have

$$
\mu_{\hat{c}_{n^{\prime}}} \nabla \Phi \rightarrow \mu_{\hat{c}} \nabla \Phi \quad \text { strongly } \quad \text { in } \quad L^{2}\left(Q_{T}\right) .
$$

Moreover, $\left(\nabla c_{n^{\prime}}-\nabla c\right) \rightarrow 0$ weakly in $L^{2}\left(Q_{T}\right)$ because of $(2.3 .25)$, thus we obtain

$$
\int_{0}^{T} \int_{\Omega} \mu_{\hat{c}_{n^{\prime}}} \nabla c_{n^{\prime}} \nabla \Phi d x d s \rightarrow \int_{0}^{T} \int_{\Omega} \mu_{\hat{c}} \nabla c \nabla \Phi d x d s .
$$


Applying the trace theorem, the last term in (2.3.27) converges too.

Thus, we can pass to the limit in (2.3.27) obtaining

$$
\begin{aligned}
-\psi(0) \int_{\Omega} c_{0} \phi(x) d x & -\int_{0}^{T} \int_{\Omega} c \psi_{s} \phi d x d s \\
& +\int_{0}^{T} \psi \int_{\Omega} \mu_{\hat{c}} \nabla c \nabla \phi d x d s+\int_{0}^{T} \psi \int_{\partial \Omega} \beta\left(c-c_{p}\right) \phi d \sigma d s=0 .
\end{aligned}
$$

Consequently

$$
\int_{0}^{T} \psi\left(\int_{\Omega} c_{s} \phi d x+\int_{\Omega} \mu_{\hat{c}} \nabla c \nabla \phi d x+\int_{\partial \Omega} \beta\left(c-c_{p}\right) \phi d \sigma\right) d s=0 .
$$

The above is true for $\phi, \psi$ satisfying (2.3.28). Therefore (2.3.28) gives, a.e in $t \in(0, T)$

$$
\left\langle\frac{d}{d t} c(t), \phi\right\rangle+a(t ; c(t), \phi)=\langle F(t), \phi\rangle \quad \forall \phi \in W_{2}^{1}(\Omega) .
$$

Since the solution of (2.3.22) is unique, we can conclude

$$
\mathcal{T} \hat{c}=c
$$

and, since the limit does not depend on the extracted sub-sequence, it follows that

$$
\mathcal{T} \hat{c}_{n} \rightarrow \mathcal{T} c
$$

weakly in $W(0, T)$ and strongly in $L^{2}\left(Q_{T}\right)$.

Now, let

$$
K:=\left\{v \in L^{2}\left(Q_{T}\right):\|v\|_{W(0, T)} \leq M\right\} .
$$

$K$ is convex and compact in $L^{2}\left(Q_{T}\right)$ and $\mathcal{T}: K \subset L^{2}\left(Q_{T}\right) \rightarrow K$ is a continuous mapping. By the Schauder fixed point theorem the proof of the existence of solutions is concluded.

\section{Proof of Proposition 2.2.2}

We prove now that $\theta \in L^{\infty}\left(Q_{T}\right)$.

To this end, we write the weak form of equation (2.1.1):

$$
\begin{array}{r}
\int_{0}^{t}\left\langle\rho \alpha \frac{\partial \theta}{\partial t}, \phi\right\rangle d s+\int_{0}^{t} \int_{\Omega} k \nabla \theta \nabla \phi d x d s+\int_{0}^{t} \int_{\partial \Omega} h\left(\theta-\theta_{\Gamma}\right) \phi d \sigma d s \\
=\int_{0}^{t}\langle B(\theta, c) \phi\rangle d s .
\end{array}
$$


Testing with the function $\phi=\left(\theta-\theta_{\Gamma}\right)^{-}=-\min \left\{\theta-\theta_{\Gamma}, 0\right\}$, through integration by parts, we obtain from the first term:

$$
\begin{aligned}
\int_{0}^{t} \rho\left\langle\alpha \frac{\partial \theta}{\partial t}, \phi\right\rangle d s \geq \rho \alpha_{1} & \int_{0}^{t}\left\langle\frac{\partial \theta}{\partial t}, \phi\right\rangle d s \\
& =\rho \alpha_{1} \int_{0}^{t}\left\langle\frac{\partial\left(\theta-\theta_{\Gamma}\right)}{\partial t}, \phi\right\rangle d s=\rho \alpha_{1} \frac{1}{2} \int_{\Omega}|\phi(t)|^{2} d x
\end{aligned}
$$

from the second term

$$
\begin{aligned}
k \int_{0}^{t} \int_{\Omega} \nabla \theta \nabla \phi d x d s=\int_{0}^{t} \int_{\left\{x \in \Omega: \theta-\theta_{\Gamma} \leq 0\right\}} \nabla \phi \nabla \phi d x d s & \\
& =\int_{0}^{t} \int_{\Omega}|\nabla \phi|^{2} d x d s \geq 0,
\end{aligned}
$$

from the third term

$$
\int_{0}^{t} \int_{\partial \Omega} h\left(\theta-\theta_{\Gamma}\right) \phi d \sigma d s=\int_{0}^{t} \int_{\left\{\sigma \in \partial \Omega: \theta-\theta_{\Gamma} \leq 0\right\}} h\left(\theta-\theta_{\Gamma}\right)^{2} d \sigma d s \geq 0 .
$$

Regarding the right-hand side of (2.3.30), we notice that if $\left(\theta-\theta_{\Gamma}\right)^{-} \neq 0$, then $\theta \leq \theta_{\Gamma}$, but in this range of temperature we know that $B(\theta, c)=0$, since $g_{i}=0$ and vice-versa: if $B(\theta, c) \neq 0$, then $\left(\theta-\theta_{\Gamma}\right)^{-}=0$. Therefore the right-hand side is always zero and we can conclude, from (2.3.30)-(2.3.33), that:

$$
\frac{1}{2} \int_{\Omega}\left|\left(\theta-\theta_{\Gamma}\right)^{-}(t)\right|^{2} d x \leq 0 \quad \forall t \in(0, T),
$$

thus

$$
\left(\theta-\theta_{\Gamma}\right)^{-}(t)=0 \quad \forall t \in(0, T) \quad \text { a.e. in } \Omega
$$

and $\theta(x, t) \geq \theta_{\Gamma}, \forall t \in(0, T)$.

A similar argument, using the test function $\phi=\left(\theta-\theta_{0}\right)^{+}$, leads to $\theta(x, t) \leq \theta_{0}(x)$ a.e. in $Q_{T}$.

Now we pass to consider the weak form of equation (2.1.2):

$$
\int_{0}^{t}\left\langle\frac{\partial c}{\partial t}, \phi\right\rangle d s+\int_{0}^{t} \int_{\Omega} \mu_{\theta, c} \nabla c \nabla \phi d x d s+\int_{0}^{t} \int_{\partial \Omega} \beta\left(c-c_{p}\right) \phi d \sigma d s=0,
$$

$\forall \phi \in L^{2}\left(0, t ; W_{2}^{1}(\Omega)\right)$ and for every $t \in(0, T)$, where $\langle$,$\rangle denotes the duality pairing$ between $W_{2}^{1}(\Omega)$ and $\left(W_{2}^{1}(\Omega)\right)^{*}$. 
Testing with the function $\phi=\left(c-C_{1}\right)^{-}=-\min \left\{c-C_{1}, 0\right\}$, through integration by parts, we get from the first term:

$$
\int_{0}^{t}\left\langle\frac{\partial c}{\partial t}, \phi\right\rangle d s=\int_{0}^{t}\left\langle\frac{\partial\left(c-C_{1}\right)}{\partial t}, \phi\right\rangle d s=\frac{1}{2} \int_{\Omega}|\phi(t)|^{2} d x
$$

from the second term

$$
\begin{aligned}
\int_{0}^{t} \int_{\Omega} \mu_{\theta, c} \nabla c \nabla \phi d x d s=\int_{0}^{t} \int_{\left\{x \in \Omega: c-C_{1} \leq 0\right\}} \mu_{\theta, c} \nabla \phi \nabla \phi d x d s & \\
& =\int_{0}^{t} \int_{\Omega} \mu_{\theta, c}|\nabla \phi|^{2} d x d s \geq 0
\end{aligned}
$$

and from the third term

$$
\begin{aligned}
\int_{0}^{t} \int_{\partial \Omega} \beta\left(c-c_{p}\right) \phi d \sigma d s=\int_{0}^{t} \int_{\left\{\sigma \in \partial \Omega: c-C_{1} \leq 0\right\}} & \beta\left(c-c_{p}\right)\left(c-C_{1}\right) d \sigma d s \\
& \geq \int_{0}^{t} \int_{\left\{\sigma \in \partial \Omega: c-C_{1} \leq 0\right\}} \beta\left(c-C_{1}\right)^{2} d \sigma d s \geq 0
\end{aligned}
$$

Thus, from (2.3.34)-(2.3.37), we obtain

$$
\frac{1}{2} \int_{\Omega}\left|\left(c-C_{1}\right)^{-}(t)\right|^{2} d x \leq 0 \quad \forall t \in(0, T),
$$

thus

$$
\left(c-C_{1}\right)^{-}(t)=0 \quad \forall t \in(0, T) \quad \text { a.e. in } \Omega
$$

and $c(x, t) \geq C_{1}, \forall t \in(0, T)$. A similar argument, using the test function $\phi=\left(c-C_{2}\right)^{+}$, leads to $c(x, t) \leq C_{2}$ a.e in $Q_{T}$.

\subsection{Proof of uniqueness}

We commence with the following regularity result:

Lemma 2.4.1. Under the assumptions of Theorem 2.2.3, the solutions $\theta$, c to the initial-boundary values problems related to equations (2.1.1)-(2.1.2) are in $W_{5}^{2,1}\left(Q_{T}\right)$.

Proof. Until now we have proved the existence of at least one solution for the initialboundary value problems related to equations (2.1.1), (2.1.2) and we proved that the 
solutions are bounded in $L^{\infty}\left(Q_{T}\right)$ (Prop. 2.2.2). Then we can directly apply a classical result from Lady̆zenskaja ([33, Theorem 10.1, Chap. 3]), which gives us that $\theta$ and $c$ are Hölder continuous functions, with Hölder exponent depending, among the others, also on the maximum of the functions over $Q_{T}$. It follows that the right-hand sides of the ODEs (2.1.3)-(2.1.4) are continuous functions, therefore the corresponding solutions, $p$ and $m$, are continuously differentiable.

Thus, the PDEs (2.1.1)-(2.1.2) have continuous coefficients and, since $\theta_{0}, c_{0} \in W_{5}^{2}(\Omega)$ and $h, \beta \in W_{5}^{1}(\partial \Omega)$, we can apply another classical result from Lady̆zenskaja ([33, Theorem 9.1, Chap. 4] ) which yields: $\theta, c \in W_{5}^{2,1}\left(Q_{T}\right)$.

Lemma 2.4.2. Assuming that $\alpha$ is constant, we have that, for every $c_{1}, c_{2} \in L^{2}\left(Q_{T}\right)$, there exists a constant $M>0$ such that, for the corresponding $\theta_{1}, \theta_{2}$, it holds:

$$
\left\|\theta_{1}-\theta_{2}\right\|_{W_{2}^{2,1}\left(Q_{T}\right)}^{2} \leq M\left\|c_{1}-c_{2}\right\|_{L^{2}\left(Q_{T}\right)}^{2} .
$$

Proof.

We consider the heat equation of our system:

$$
\rho \alpha \frac{\partial \theta}{\partial t}=k \Delta \theta+\rho L_{p} p_{t}+\rho L_{m} m_{t}
$$

We write (2.4.2) for $\theta_{1}, c_{1}, p_{1}, m_{1}$ and $\theta_{2}, c_{2}, p_{2}, m_{2}$. Subtracting and denoting as usual $\theta=\theta_{1}-\theta_{2}$, we see that the difference satisfies the following system:

$$
\begin{aligned}
\rho \alpha \frac{\partial \theta}{\partial t}-k \Delta \theta & =\rho\left(L_{p}\left(\theta_{1}\right) p_{1, t}-L_{p}\left(\theta_{2}\right) p_{2, t}\right)+\rho\left(L_{m}\left(\theta_{1}\right) m_{1, t}-L_{m}\left(\theta_{2}\right) m_{2, t}\right)=: f \\
-k \frac{\partial \theta}{\partial \nu} & =h \theta \\
\theta(x, 0) & =0 .
\end{aligned}
$$

Applying again standard parabolic theory (cf.[37, Theorem 6.2, Chap. 4] ), we know that there exists a positive constant $K$ such that we can estimate the norm of the solution as follows:

$$
\|\theta\|_{W_{2}^{2,1}\left(Q_{T}\right)} \leq K\|f\|_{L^{2}\left(Q_{T}\right)}
$$

Now we can estimate the term $\|f\|_{L^{2}\left(Q_{T}\right)}$, by means of Lemma 2.3.1 (b) and assumptions (A2), as:

$$
\begin{array}{r}
\left\|\rho\left(L_{p}\left(\theta_{1}\right) p_{1, t}-L_{p}\left(\theta_{2}\right) p_{2, t}\right)+\rho\left(L_{m}\left(\theta_{1}\right) m_{1, t}-L_{m}\left(\theta_{2}\right) m_{2, t}\right)\right\|_{L^{2}\left(Q_{T}\right)} \\
\leq K_{1}\left\|\theta_{1}-\theta_{2}\right\|_{L^{2}\left(Q_{T}\right)}+K_{2}\left\|c_{1}-c_{2}\right\|_{L^{2}\left(Q_{T}\right)}
\end{array}
$$

with $K_{1}, K_{2}$ positive constants. Now, using Lemma 2.3.3, we can estimate the term $\left\|\theta_{1}-\theta_{2}\right\|_{L^{2}\left(Q_{T}\right)}$ and therewith finish the proof.

Lemma 2.4.3. Let $u \in L^{\infty}\left(0, T ; L^{2}(\Omega)\right) \cap L^{2}\left(0, T ; W_{2}^{1}(\Omega)\right)$, then there holds

$$
\int_{0}^{T}\|u(t)\|_{L^{10 / 3}(\Omega)}^{10 / 3} d t \leq\left(\int_{0}^{T}\|u(t)\|_{L^{6}(\Omega)}^{2} d t\right)\|u\|_{L^{\infty}\left(0, T ; L^{2}(\Omega)\right)}^{4 / 3} .
$$


Proof. Owing to Riesz' convexity theorem (cf. [8, Oss. 2, Chap. 4]), we have

$$
\|u\|_{L^{r}(\Omega)} \leq\|u\|_{L^{q_{1}(\Omega)}}^{1-\Theta}\|u\|_{L^{q_{2}(\Omega)}}^{\Theta},
$$

for all $u \in L^{q_{1}}(\Omega) \cap L^{q_{2}}(\Omega)$ with $1 \leq q_{1}, q_{2}<\infty, 0<\Theta<1$, and $\frac{1}{r}=\frac{1-\Theta}{q_{1}}+\frac{\Theta}{q_{2}}$. Invoking the continuous embedding $W_{2}^{1}(\Omega) \subset L^{6}(\Omega)$, the assertion follows by defining $q_{1}=6, q_{2}=2, \Theta=\frac{2}{5}$, and $r=\frac{10}{3}$.

We are now in a position to prove Theorem 2.2.3.

Proof of Theorem 2.2.3

We write equation (2.1.2) for $c_{1}$ and $c_{2}$, subtract, integrate over $Q_{T}$ and test by $c_{1}-c_{2}$. In the sequel we will use the following notations: $c=c_{1}-c_{2}, \theta=\theta_{1}-\theta_{2}, p=$ $p_{1}-p_{2}, m=m_{1}-m_{2}$.

We have:

$$
\begin{aligned}
\frac{1}{2} \int_{\Omega} c^{2}(t) d x & +\int_{0}^{t} \int_{\Omega}\left(\left(1-p_{1}-m_{1}\right) D\left(\theta_{1}\right) \nabla c_{1}-\left(1-p_{2}-m_{2}\right) D\left(\theta_{2}\right) \nabla c_{2}\right) \nabla c d x d s \\
& +\int_{0}^{t} \int_{\partial \Omega} \beta c^{2} d \sigma d s=0 .
\end{aligned}
$$

Now,

$$
\begin{aligned}
\int_{0}^{t} \int_{\Omega}((1- & \left.\left.p_{1}-m_{1}\right) D\left(\theta_{1}\right) \nabla c_{1}-\left(1-p_{2}-m_{2}\right) D\left(\theta_{2}\right) \nabla c_{2}\right) \nabla c d x d s \\
& =\int_{0}^{t} \int_{\Omega}\left(1-p_{1}-m_{1}\right) D\left(\theta_{1}\right)|\nabla c|^{2} d x d s-\int_{0}^{t} \int_{\Omega}(p+m) D\left(\theta_{1}\right) \nabla c_{2} \nabla c d x d s \\
& +\int_{0}^{t} \int_{\Omega}\left(1-p_{2}-m_{2}\right)\left(D\left(\theta_{1}\right)-D\left(\theta_{2}\right)\right) \nabla c_{2} \nabla c d x d s .
\end{aligned}
$$

Denoting, in the sequel, by $K_{i}$ generic positive constants independent of $\theta$ and $c$, we obtain:

$$
\begin{aligned}
\frac{1}{2} \int_{\Omega} c^{2}(t) d x & +K_{1} \int_{0}^{t}\|\nabla c\|_{L^{2}(\Omega)}^{2} d x d s \\
& \leq \int_{0}^{t} \int_{\Omega}|p+m|\left|D\left(\theta_{1}\right)\right|\left|\nabla c_{2}\right||\nabla c| d x d s \\
& +\int_{0}^{t} \int_{\Omega}\left|1-p_{2}-m_{2}\right|\left|D\left(\theta_{1}\right)-D\left(\theta_{2}\right)\right|\left|\nabla c_{2}\right||\nabla c| d x d s .
\end{aligned}
$$


By means of Lemma 2.4.1, we know that $c_{2} \in W_{5}^{2,1}\left(Q_{T}\right)$. According to Amann (cf [1, Theorem 1.1], Theorem 1.1), we have the embedding $W_{5}^{2,1}\left(Q_{T}\right) \hookrightarrow C\left([0, T] ; W_{5}^{1}(\Omega)\right)$. Thus, we can estimate the first term in the right hand-side of (2.4.4) as:

$$
\begin{aligned}
& \int_{0}^{t} \int_{\Omega}\left|p+m \| D\left(\theta_{1}\right)\right|\left|\nabla c_{2}\right||\nabla c| d x d s \\
& \leq \int_{0}^{t}\|p+m\|_{L^{10 / 3}(\Omega)}\left\|\nabla c_{2}\right\|_{L^{5}(\Omega)}\left\|D\left(\theta_{1}\right)\right\|_{L^{\infty}(\Omega)}\|\nabla c\|_{L^{2}(\Omega)} d s \\
& \leq \frac{K_{1}}{4} \int_{0}^{t}\|\nabla c\|_{L^{2}(\Omega)}^{2} d s+\frac{4 K_{2}}{K_{1}} \int_{0}^{t}\|p+m\|_{L^{10 / 3}(\Omega)}^{2} d s .
\end{aligned}
$$

Moreover, thanks to Lemma 2.3.1 (b), we get:

$$
\begin{aligned}
& \int_{0}^{t}\|p+m\|_{L^{10 / 3}(\Omega)}^{2} d s=\int_{0}^{t}\left[\int_{\Omega}|p+m|^{10 / 3} d x\right]^{3 / 5} d s \\
& \leq K_{3} \int_{0}^{t}\left[\int_{0}^{s} \int_{\Omega} \theta^{10 / 3} d x d \tau\right]^{3 / 5} d s+K_{4} \int_{0}^{t}\left[\int_{0}^{s} \int_{\Omega} c^{10 / 3} d x d \tau\right]^{3 / 5} d s .
\end{aligned}
$$

Now, we apply Lemma 2.4.3, Young's inequality and the embedding $W_{2}^{1}(\Omega) \hookrightarrow L^{6}(\Omega)$ to the right-hand side of (2.4.6), obtaining:

$$
\begin{aligned}
& \int_{0}^{t}\left[\int_{0}^{s} \int_{\Omega} \theta^{10 / 3} d x d \tau\right]^{3 / 5} d s \leq \int_{0}^{t}\left[\int_{0}^{s} K_{5}\|\theta\|_{W_{2}^{1}(\Omega)}^{2} d \tau\right]^{3 / 5}\|\theta\|_{L^{\infty}\left(0, s ; L^{2}(\Omega)\right)}^{4 / 5} d s \\
& \leq \frac{3 K_{5}}{5} \int_{0}^{t} \int_{0}^{s}\|\theta\|_{W_{2}^{1}(\Omega)}^{2} d \tau d s+\frac{2}{5} \int_{0}^{t}\|\theta\|_{L^{\infty}\left(0, s ; L^{2}(\Omega)\right)}^{2} d s .
\end{aligned}
$$

Analogously, it holds:

$$
\int_{0}^{t}\left[\int_{0}^{s} \int_{\Omega} c^{10 / 3} d x d \tau\right]^{3 / 5} d s \leq K_{5} \frac{3}{5} \int_{0}^{t} \int_{0}^{s}\|c\|_{W_{2}^{1}(\Omega)}^{2} d \tau d s+\frac{2}{5} \int_{0}^{t}\|c\|_{L^{\infty}\left(0, s ; L^{2}(\Omega)\right)}^{2} d s(2.4 .8)
$$

Regarding the second term in the right-hand side of (2.4.4), using Lemma 4.1, assumption (A6) and Young's inequality again, we have:

$$
\begin{aligned}
\int_{0}^{t} \int_{\Omega} \mid 1-p_{2}-m_{2} \| D\left(\theta_{1}\right) & -D\left(\theta_{2}\right)\left\|\nabla c_{2}|| \nabla c \mid d x d s \leq\right\| \nabla c_{2}\left\|_{L^{\infty}\left(0, t ; L^{3}(\Omega)\right)} \int_{0}^{t}\right\| \theta\left\|_{L^{6}(\Omega)}\right\| \nabla c \|_{L^{2}(\Omega)} d s \\
& \leq K_{6} \int_{0}^{t}\|\theta\|_{W_{2}^{1}(\Omega)}^{2} d s+\frac{K_{1}}{4} \int_{0}^{t}\|\nabla c\|_{L^{2}(\Omega)}^{2} d s .
\end{aligned}
$$


Using (5.4) to (2.4.9), we find that

$$
\begin{aligned}
& \min \left\{\frac{1}{2}, \frac{K_{1}}{2}\right\} \int_{\Omega} c^{2}(t) d x+\int_{0}^{t}\|\nabla c\|_{L^{2}(\Omega)}^{2} d s \\
& \leq K_{7}\left(\int_{0}^{t}\|\theta\|_{W_{2}^{1}(\Omega)}^{2} d s+\int_{0}^{t}\|\theta\|_{L^{\infty}\left(0, s ; L^{2}(\Omega)\right)}^{2} d s\right)+\frac{3 K_{5}}{5} \int_{0}^{t} \int_{0}^{s}\|\theta\|_{W_{2}^{1}(\Omega)}^{2} d \tau d s \\
& +K_{8}\left(\int_{0}^{t} \int_{0}^{s}\|c\|_{W_{2}^{1}(\Omega)}^{2} d \tau d s+\int_{0}^{t}\|c\|_{L^{\infty}\left(0, s ; L^{2}(\Omega)\right)}^{2} d s\right) .
\end{aligned}
$$

Thanks to the embedding $W_{2}^{2,1}\left(Q_{T}\right) \hookrightarrow C\left([0, T] ; W_{2}^{1}(\Omega)\right)$ (see [2], Theorem 1.1) and Lemma 5.2, we obtain for the first term in the right-hand side of (2.4.10):

$$
\int_{0}^{t}\|\theta(s)\|_{W_{2}^{1}(\Omega)}^{2} d s \leq \int_{0}^{t}\|\theta\|_{L^{\infty}\left(0, s ; W_{2}^{1}(\Omega)\right)}^{2} d s \leq K_{9} \int_{0}^{t}\|\theta\|_{W_{2}^{2,1}\left(Q_{s}\right)} d s \leq \int_{0}^{t} K_{10} \int_{0}^{s}\|c\|_{L^{2}(\Omega)}^{2} d \tau d s
$$

Analogous estimates hold for the other terms involving $\theta$. Thus we end up with:

$$
\begin{aligned}
\|c(t)\|_{L^{2}(\Omega)}^{2} & +\int_{0}^{t}\|\nabla c\|_{L^{2}(\Omega)}^{2} d s \leq K_{11} \int_{0}^{t}\|c\|_{L^{\infty}\left(0, s ; L^{2}(\Omega)\right)}^{2} d s \\
& +K_{12} \int_{0}^{t} \int_{0}^{s}\|\nabla c\|_{L^{2}(\Omega)}^{2} d \tau d s+K_{13} \int_{0}^{t} \int_{0}^{s}\|c\|_{L^{2}(\Omega)}^{2} d \tau d s \quad \forall t \in[0, T] .
\end{aligned}
$$

Taking the essential supremum over $[0, \hat{t}]$ for some $\hat{t} \in[0, T]$ in the previous inequality, we obtain:

$$
\begin{gathered}
\|c(t)\|_{L^{\infty}\left(0, \hat{t} L^{2}(\Omega)\right)}^{2}+\int_{0}^{\hat{t}}\|\nabla c\|_{L^{2}(\Omega)}^{2} \leq K_{11} \int_{0}^{\hat{t}}\|c\|_{L^{\infty}\left(0, s ; L^{2}(\Omega)\right)}^{2} d s \\
+K_{12} \int_{0}^{\hat{t}} \int_{0}^{s}\|\nabla c\|_{L^{2}(\Omega)}^{2} d \tau d s+K_{13} \int_{0}^{\hat{t}} \int_{0}^{s}\|c\|_{L^{2}(\Omega)}^{2} d \tau d s .
\end{gathered}
$$

Since $\int_{0}^{\hat{t}} \int_{0}^{s}\|c\|_{L^{2}(\Omega)}^{2} d \tau d s \leq T \int_{0}^{\hat{t}}\|c\|_{L^{\infty}\left(0, s ; L^{2}(\Omega)\right)}^{2} d s$, we can apply the Gronwall lemma and conclude the proof of Theorem 3.2. 


\section{Chapter 3}

\section{Analysis of a related quasilinear parabolic system}

\subsection{Problem statement and strategy}

The uniqueness result presented in the previous chapter has been obtained under quite strong assumptions. This leads us to consider a related system constituted of the two quasilinear parabolic equations, with the same structure of those present in the complete model, under weaker assumptions, not coupled though with the two additional ODEs for the phase transitions. It seems, in fact, that the analysis performed in this chapter can't be carried over to the whole system considered previously. Nevertheless we believe in the intrinsic mathematical interest of this chapter, dealing with a quite general and relevant class of parabolic systems, often encountered in problems arising from physics. Very similar systems of quasilinear parabolic equations are studied also for other applications (see for instance, [42] and [43]).

Here we examine, in the domain $Q_{T}=\Omega \times(0, T), \Omega \subset \mathbb{R}^{N}$, the following system of equations:

$$
\begin{aligned}
\theta_{t}-\Delta \theta & =r(\theta, c) \\
c_{t}-\operatorname{div}(D(\theta, c) \nabla c) & =0
\end{aligned}
$$

with boundary conditions

$$
\begin{aligned}
\frac{\partial \theta}{\partial \nu}+h\left(x, \theta, \theta_{\Gamma}(x, t)\right) & =0 \\
-D(\theta, c) \frac{\partial c}{\partial \nu} & =b(x, t)
\end{aligned}
$$

and initial conditions

$$
\begin{aligned}
& \theta(x, 0)=\theta^{0}(x) \\
& c(x, 0)=c^{0}(x) .
\end{aligned}
$$

The properties of the nonlinearities $r, D$, and $h$, as well as the hypotheses on the data $b, \theta_{\Gamma}, \theta^{0}$, and $c^{0}$ will be specified in the next section. 
This system presents differences from the one studied in the previous chapter, mainly in the nonlinear boundary condition for $\theta$, in the boundary condition for $c$ and in the fact that we allow the coefficient $D$ to depend both on $\theta$ and $c$. Hence, in comparison with the analysis contained in Chapter 2, we conduct here a different proof of existence of solutions and of uniqueness as well.

The first part of our analysis aims to show the existence of a generalized solution $(\theta, c)$ of the system (3.1.1)-(3.1.6), under appropriate regularity assumptions, with

$$
\theta \in L^{\infty}\left(0, T ; H^{1}(\Omega)\right) \cap H^{1}\left(0, T ; L^{2}(\Omega)\right), \quad c \in L^{2}\left(0, T ; H^{1}(\Omega)\right) \cap H^{1}\left(0, T ;\left(H^{1}(\Omega)\right)^{\prime}\right) .
$$

Together with the existence, employing a maximum principle and a Moser iteration, we also prove that the solution $\theta(x, t)$ is positive and uniformly bounded from above in $Q_{T}$, only assuming linear growth of $r(\theta, c)$.

Then we come to the discussion of the more delicate question of uniqueness. After the usual observation that, in order to prove a uniqueness result, it is useful to proceed with analyzing the regularity of solutions, we address explicitly the question of regularity. To the subject of nonlinear parabolic equations of second order are dedicated many monographs, among the quite recent ones, we mention [1], [31] and [35]. Since it seemed to us that no complete result is available in literature for the case of system (3.1.1)(3.1.6), we develop a strategy technically involved but self contained and elementary as far as possible. We are able to show that, for $N \leq 3, \theta$ possesses the regularity

$$
\nabla \theta \in L^{2}\left(0, T ; L^{\infty}(\Omega) \cap C^{\alpha}(\Omega)\right),
$$

for some $\alpha>0$. To this aim, we proceed in several steps. Due to the nonlinearity in the boundary condition (3.1.3), we first regularize the boundary condition with a parameter $\delta>0$ that we will eventually let tend to zero. A fundamental tool employed in our approach to the problem are the anisotropic Sobolev spaces, which are found to be particularly adequate in the treatment of equations presenting different regularity in tangential and in normal directions, that is the case of our system with associated third-order boundary conditions.

Regarding the assumptions, we notice that no assumption about the regularity of the gradient of the solution, in particular its boundedness, was made. This technique is inspired from the estimation technique proposed in [40] for elliptic equations with linear boundary conditions. Here, however, we obtain different estimates in tangential and normal directions and it is necessary to use an embedding theorem for anisotropic spaces that can be found in a separate section, proved by the methods of Besov ([6]). The last part contains the proof of uniqueness and stability for the whole system. We prove uniqueness and continuous dependence on the data, $\theta_{\Gamma}, b, \theta^{0}$ and $c^{0}$. The proof is based on an $L^{p}$-variant of the Gronwall lemma. If the standard Gronwall lemma can be viewed as a result of the fact that the $L^{\infty}$-norm of the function $v$ is bounded above by its weighted $L^{1}$-norm, what we show is that an $L^{p}$-Gronwall estimate still holds if the $L^{\infty}$-norm on the left-hand side is replaced with an $L^{p}$-norm for $p>1$.

Remark 2. Although it is not possible to describe the whole process of case hardening through this system, still the stages without phase fractions growth may be therewith 
correctly modelled. In this scenario, the interaction between temperature evolution and diffusion of carbon would be reflected in the carbon diffusion coefficient $D(\theta, c)$ and in the heat source term $r(\theta, c)$. The function $\theta_{\Gamma}$ would be again the external temperature of the atmosphere in the furnace and the flux of carbon through the surface of the workpiece would be expressed by the function $b(x, t)$. In this contest we point out that a relevant issue for applications is the fact that the boundary condition for the $\theta$, if interpreted as temperature, encompasses heat exchanges by conduction, convection and radiation. This is also a reason why we require no growth restriction on $h\left(x, \theta, \theta_{\Gamma}\right)$, and the boundary condition (3.1.3) thus includes thus the case

$$
\frac{\partial \theta}{\partial \nu}+\alpha(x)\left(\theta-\theta_{\Gamma}\right)+\beta(x)\left(\theta^{4}-\theta_{\Gamma}^{4}\right)=0,
$$

with coefficients $\alpha(x), \beta(x) \geq 0, \alpha(x)+\beta(x) \geq \alpha_{0}>0$.

\subsection{Main results}

We state for system (3.1.1)-(3.1.6) two sets of hypotheses: Hypothesis 3.2.1 for existence and its stronger version 3.2.2 for regularity and uniqueness. Note that we do not assume any upper bound for the growth of $h$.

Hypothesis 3.2.1. The domain $\Omega \subset \mathbb{R}^{N}, N \leq 3$, is bounded and has Lipschitzcontinuous boundary.

We prescribe the data $b \in L^{2}(\partial \Omega \times(0, T)), c^{0} \in L^{2}(\Omega), \theta^{0} \in V \cap L^{\infty}(\Omega)$, and let $0<\theta_{*}:=\operatorname{essinf} \theta^{0}$.

The function $h$ is measurable in $x$, locally Lipschitz-continuous with respect to $\theta$ and Lipschitz-continuous with respect to $\theta_{\Gamma}$, with the properties

$$
\begin{gathered}
h\left(x, \theta_{*}, \theta_{\Gamma}\right) \leq 0 \\
\exists a>0: \forall m>0 \exists C_{m}>0: \theta_{\Gamma} \leq m, \theta \geq 0 \Rightarrow h\left(x, \theta, \theta_{\Gamma}\right) \geq a \theta-C_{m} .
\end{gathered}
$$

Furthermore,

- $\theta_{\Gamma} \in L^{\infty}(\partial \Omega \times(0, T)),\left(\theta_{\Gamma}\right)_{t} \in L^{2}(\partial \Omega \times(0, T)), \theta_{\Gamma} \geq \theta_{*}$ a. e.,

- $r$ is Lipschitz-continuous with respect to both variables, $0 \leq r$

- $D$ is continuous and there exist constants $d_{0}, d_{1}$ such that

$$
0<d_{0} \leq D(\theta, c) \leq d_{1}
$$

Hypothesis 3.2.2. In addition to Hypothesis 3.2.1, we assume that the domain $\Omega$ is of class $C^{2,1}$, that is, the outward normal vector has Lipschitz-continuous derivatives until the second order. There exist connected relatively open subsets $\Gamma_{j}$ of $\partial \Omega, j=1, \ldots, n$, $\Gamma_{i} \cap \Gamma_{j}=\emptyset, i \neq j$, which are $C^{2,1}$-diffeomorphic to open bounded subsets of $\mathbb{R}^{2}$, and a function $h_{0} \in W^{2, \infty}(\partial \Omega)$ such that $h\left(x, \theta, \theta_{\Gamma}\right)=h_{0}(x)\left(\theta-\theta_{\Gamma}\right)$ on $\partial \Omega \backslash \bigcup_{j=1}^{n} \Gamma_{j}$. Furthermore, 


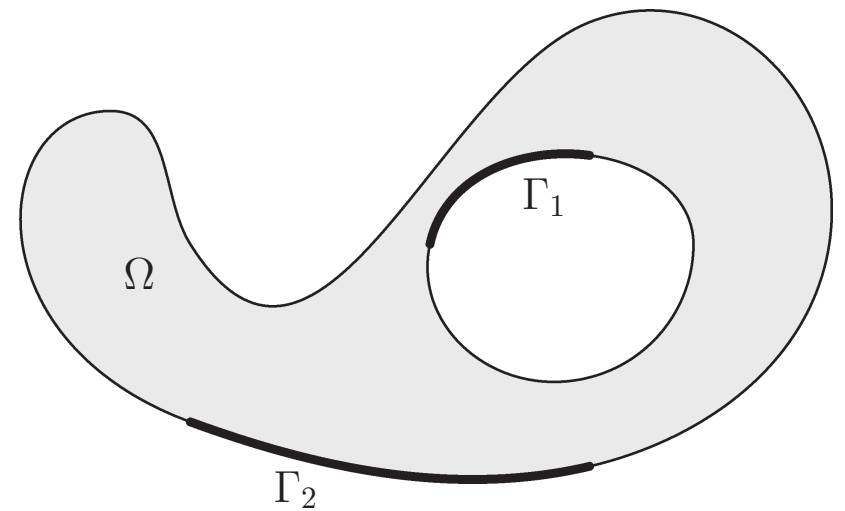

Figure 3.1: An admissible domain $\Omega$. Thick lines denote the set of nonlinear boundary conditions.

$-\theta^{0} \in W^{2,2}(\Omega)$,

- $h$ is of class $W_{l o c}^{2, \infty}$ with respect to all variables,

- $\theta_{\Gamma} \in L^{2}\left(0, T ; W^{2,2}(\partial \Omega)\right),\left(\theta_{\Gamma}\right)_{t} \in L^{2}\left(0, T ; W^{1,2}(\partial \Omega)\right)$,

- r, F, $\partial_{\theta} F$ are globally Lipschitz-continuous with respect to both variables $\theta$ and $c$, where we set

$$
F(\theta, c)=\int_{0}^{c} D\left(\theta, c^{\prime}\right) \mathrm{d} c^{\prime}
$$

- $\partial_{\theta} F$ is globally bounded.

We deal with the following weak formulation of (3.1.1)-(3.1.4).

$$
\begin{array}{r}
\int_{\Omega}\left(\theta_{t} \varphi+\nabla \theta \cdot \nabla \varphi-r(\theta, c) \varphi\right) \mathrm{d} x+\int_{\partial \Omega}\left(h\left(x, \theta, \theta_{\Gamma}(x, t)\right) \varphi \mathrm{d} S=0\right. \\
\int_{\Omega}\left(c_{t} \psi+D(\theta, c) \nabla c \cdot \nabla \psi\right) \mathrm{d} x+\int_{\partial \Omega} b(x, t) \psi \mathrm{d} S=0
\end{array}
$$

for every test functions $\varphi, \psi \in V$.

Theorem 3.2.3. (Existence) Let Hypothesis 3.2.1 hold. Then there exists $K_{0}>0$ and a solution $(\theta, c)$ to the system (3.2.1)-(3.2.2) with initial conditions (3.1.5)-(3.1.6), with the regularity $\theta \in L^{\infty}(0, T ; V), \theta_{t} \in L^{2}\left(Q_{T}\right), c \in L^{2}(0, T ; V), c_{t} \in L^{2}\left(0, T ; V^{\prime}\right)$, and such that $\theta_{*} \leq \theta(x, t) \leq K_{0}$ a.e.

Theorem 3.2.4. (Regularity) Let Hypothesis 3.2.2 hold. Then every solution $(\theta, c)$ to (3.2.1)-(3.2.2) from Theorem 3.2.3 has the additional regularity $\nabla \theta \in L^{2}\left(0, T ; L^{\infty}(\Omega)\right)$. 
To simplify the notation, we introduce the symbol

$$
|w(t)|_{p}=\left(\int_{\Omega}|w(x, t)|^{p} \mathrm{~d} x\right)^{1 / p} \quad \text { for } t \in(0, T),
$$

to denote the partial $L^{p}(\Omega)$-norm of a generic function $w: Q_{T} \rightarrow \mathbb{R}^{d}, d \geq 1$, with an obvious modification for $p=\infty$.

An important achievement of this chapter is the following uniqueness and continuous dependence result. It will be based on the partial Kirchhoff transform

$$
u=F(\theta, c)
$$

with $F$ from Hypothesis 3.2.2.

Theorem 3.2.5. (Uniqueness and continuous data dependence) Let Hypothesis 3.2.2 hold, and let $\left(\theta_{1}, c_{1}\right),\left(\theta_{2}, c_{2}\right)$ be two solutions with the regularity from Theorem 3.2.4 corresponding to the same $h(x, \cdot)$, and to different data $\theta_{i}^{0}, c_{i}^{0}, \theta_{\Gamma i}, b_{i}, i=1,2$, satisfying Hypothesis 3.2.2. Let $u_{i}=F\left(\theta_{i}, c_{i}\right), i=1,2$, be defined by the Kirchhoff transform (3.2.4). $\operatorname{Set} \bar{\theta}=\theta_{1}-\theta_{2}, \bar{u}=u_{1}-u_{2}, \bar{c}^{0}=c_{1}^{0}-c_{2}^{0}, \bar{\theta}^{0}=\theta_{1}^{0}-\theta_{2}^{0}, \bar{\theta}_{\Gamma}=\theta_{\Gamma 1}-\theta_{\Gamma 2}, \bar{b}=b_{1}-b_{2}$. Then there exists a positive constant $M>0$ depending only on $\theta_{1}^{0}, c_{1}^{0}, \theta_{\Gamma 1}, b_{1}$, such that the inequality

$$
|\bar{\theta}(t)|_{2}^{2}+\left|\nabla \int_{0}^{t} \bar{u}(\tau) \mathrm{d} \tau\right|_{2}^{2}+\int_{0}^{t}\left(|\nabla \bar{\theta}|_{2}^{2}+|\bar{u}|_{2}^{2}\right)(\tau) \mathrm{d} \tau \leq M \alpha(t),
$$

holds for every $t \in[0, T]$, where we set

$$
\alpha(t)=\left|\bar{\theta}^{0}\right|_{2}^{2}+\left|\bar{c}^{0}\right|_{2}^{2}+\int_{0}^{t} \int_{\partial \Omega}\left|\bar{\theta}_{\Gamma}(x, \tau)\right|^{2} \mathrm{~d} S \mathrm{~d} \tau+\int_{0}^{t} \int_{\partial \Omega}|\bar{b}(x, \tau)|^{2} \mathrm{~d} S \mathrm{~d} \tau .
$$

\subsection{Proof of existence}

We fix some $K>0$ that will be specified later, and set

$$
h_{K}\left(x, \theta, \theta_{\Gamma}\right)=h\left(x, \max \left\{\theta_{*}, \min \{\theta, K\}\right\}, \theta_{\Gamma}\right) .
$$

Instead of (3.2.1)-(3.2.2), we consider the decoupled and truncated problem

$$
\begin{aligned}
\int_{\Omega}\left(\theta_{t} \varphi+\nabla \theta \cdot \nabla \varphi-r(\theta, \hat{c}) \varphi\right) \mathrm{d} x+\int_{\partial \Omega} h_{K}\left(x, \theta, \theta_{\Gamma}(x, t)\right) \varphi \mathrm{d} S & =0 \\
\int_{\Omega}\left(c_{t} \psi+D(\hat{\theta}, \hat{c}) \nabla c \cdot \nabla \psi\right) \mathrm{d} x+\int_{\partial \Omega} b(x, t) \psi \mathrm{d} S & =0
\end{aligned}
$$

for every test functions $\varphi, \psi \in V$, with given functions $\hat{\theta}, \hat{c} \in L^{2}\left(Q_{T}\right)$, and with initial conditions (3.1.5)-(3.1.6). We now use the Schauder fixed point theorem. For $m_{c}, m_{\theta}>0$, we fix the set

$$
Z\left(m_{\theta}, m_{c}\right)=\left\{(\theta, c) \in L^{2}\left(Q_{T}\right) \times L^{2}\left(Q_{T}\right): \int_{0}^{T}|\theta(t)|_{2}^{2} \mathrm{~d} t \leq m_{\theta}, \int_{0}^{T}|c(t)|_{2}^{2} \mathrm{~d} t \leq m_{c}\right\} .
$$


Approximating the functions $\theta$ and $c$ by Faedo-Galerkin expansions into the system of eigenfunctions of the Laplacian with homogeneous Neumann boundary conditions (see [36]), we obtain by compactness argument the existence of solutions to (3.3.1)-(3.3.2), as well as the estimates

$$
\begin{gathered}
\int_{0}^{T}\left(\left|\theta_{t}(t)\right|_{2}^{2}+|\Delta \theta(t)|_{2}^{2}\right) \mathrm{d} t+\sup _{t \in(0, T)}|\nabla \theta(t)|_{2}^{2} \leq R_{1}\left(1+\int_{0}^{T}|\hat{c}(t)|_{2}^{2} \mathrm{~d} t\right) \\
\int_{0}^{T}\left(\left|c_{t}(t)\right|_{V^{\prime}}^{2}+|\nabla c(t)|_{2}^{2}\right) \mathrm{d} t+\sup _{t \in(0, T)}|c(t)|_{2}^{2} \leq R_{2},
\end{gathered}
$$

where $R_{1}, R_{2}$ are constants independent of $\hat{\theta}$ and $\hat{c}$. It now suffices to choose $m_{c}=$ $T R_{2}$ and $m_{\theta}=T R_{1}\left(1+T R_{2}\right)$ to check that the solution $(\theta, c)$ belongs to $Z\left(m_{\theta}, m_{c}\right)$ whenever $(\hat{\theta}, \hat{c}) \in Z\left(m_{\theta}, m_{c}\right) . Z\left(m_{\theta}, m_{c}\right)$ is closed, convex and the solution mapping associated with (3.3.1)-(3.3.2) is compact in $L^{2}\left(Q_{T}\right) \times L^{2}\left(Q_{T}\right)$, hence it admits a fixed point, which is a solution to $(3.2 .1)-(3.2 .2)$ with $h$ replaced by $h_{K}$.

It remains to find uniform bounds $\theta_{*} \leq \theta \leq K_{0}$ independent of $K$. Choosing $K>K_{0}$, we eventually obtain the assertion.

To do so, we first choose in (3.3.1) $\varphi=-\left(\theta_{*}-\theta\right)^{+}$, where $z^{+}$denotes the positive part of an element $z \in \mathbb{R}$. We obtain

$$
\frac{1}{2} \frac{\mathrm{d}}{\mathrm{d} t} \int_{\Omega}\left|\left(\theta_{*}-\theta\right)^{+}\right|^{2} \mathrm{~d} x+\int_{\Omega}\left|\nabla\left(\theta_{*}-\theta\right)^{+}\right|^{2} \mathrm{~d} x \leq 0
$$

hence $\theta(x, t) \geq \theta_{*}$ a. e.

The upper bound is obtained by Moser iterations as in [33]. Set $f(x, t)=r(\theta(x, t), c(x, t))$ and $\theta_{K}=\min \{\theta, K\}$. Estimates (3.3.4)-(3.3.3), Sobolev embeddings, and interpolations in Lebesgue spaces yield $f \in L^{2}\left(0, T ; L^{6}(\Omega)\right) \cap L^{\infty}\left(0, T ; L^{2}(\Omega)\right) \subset L^{q}\left(Q_{T}\right)$ for $q=10 / 3$.

The function $\theta$ is a solution of the equation

$$
\int_{\Omega}\left(\theta_{t} \varphi+\nabla \theta \cdot \nabla \varphi-f(x, t) \varphi\right) \mathrm{d} x+\int_{\partial \Omega} h\left(x, \theta_{K}, \theta_{\Gamma}(x, t)\right) \varphi \mathrm{d} S=0
$$

for every $\varphi \in V$. We may choose in particular $\varphi=p \theta_{K}^{p-1}$ for $p>1$, with the intention to let $p$ tend to $\infty$. In the remaining part of this section, we denote by $C$ any constant independent of $K$ and $p$. Setting $v_{K p}=\theta_{K}^{p / 2}$, we obtain from (3.3.5) after integration with respect to $t$ that

$$
\begin{aligned}
& \left|v_{K p}(t)\right|_{2}^{2}+\int_{0}^{t}\left|\nabla v_{K p}(\tau)\right|_{2}^{2} \mathrm{~d} \tau+a p \int_{0}^{t} \int_{\partial \Omega}\left|v_{K p}(x, \tau)\right|^{2} \mathrm{~d} S \mathrm{~d} \tau \\
& \leq C^{p}+C p\left(\int_{0}^{t} \int_{\Omega}|f|\left|v_{K p}\right|^{2 / p^{\prime}} \mathrm{d} x \mathrm{~d} \tau+\int_{0}^{t} \int_{\partial \Omega}\left|v_{K p}(x, \tau)\right|^{2 / p^{\prime}} \mathrm{d} S \mathrm{~d} \tau\right)
\end{aligned}
$$

where prime denotes here and in the sequel the conjugate exponent. Using Hölder's inequality, we eliminate the boundary integrals and obtain

$$
\begin{aligned}
\left|v_{K p}(t)\right|_{2}^{2}+\int_{0}^{t}\left|\nabla v_{K p}(\tau)\right|_{2}^{2} \mathrm{~d} \tau & \leq C^{p}+C p \int_{0}^{t} \int_{\Omega}|f|\left|v_{K p}\right|^{2 / p^{\prime}}(x, \tau) \mathrm{d} x \mathrm{~d} \tau(3.3 \\
& \leq C^{p}+C p\|f\|_{q}\left\|v_{K p}\right\|_{2 q^{\prime}}^{2 / p^{\prime}}
\end{aligned}
$$


where we use for simplicity the notation

$$
\|v\|_{r}=\left(\int_{0}^{T} \int_{\Omega}|v(x, t)|^{r} \mathrm{~d} x \mathrm{~d} t\right)^{1 / r}
$$

for $v \in L^{r}(\Omega \times(0, T))$ and $r \geq 1$. Set $q_{0}=(N / 2)+1$. Then $q_{0}<q$ and we define $\varrho>0$ by the formula $q_{0}^{\prime}=(1+\varrho) q^{\prime}$. From the Gagliardo-Nirenberg inequality we obtain the estimate

$$
\left\|v_{K p}\right\|_{2 q_{0}^{\prime}}^{2} \leq C\left(\sup _{t \in(0, T)}\left|v_{K p}(t)\right|_{2}^{2}+\int_{0}^{T}\left|\nabla v_{K p}(\tau)\right|_{2}^{2} \mathrm{~d} \tau\right)
$$

hence, by virtue of (3.3.7) and Young's inequality, we obtain

$$
\left\|v_{K p}\right\|_{2 q_{0}^{\prime}}^{2} \leq C p \max \left\{1, C^{p},\left\|v_{K p}\right\|_{2 q^{\prime}}^{2}\right\},
$$

that is,

$$
\left\|\theta_{K}\right\|_{p q_{0}^{\prime}} \leq(C p)^{1 / p} \max \left\{C,\left\|\theta_{K}\right\|_{p q^{\prime}}\right\},
$$

with a constant $C$ independent of $K$ and $p$. We now set $p_{j}=(1+\varrho)^{j}, z_{j}=\left\|\theta_{K}\right\|_{p_{j} q_{0}^{\prime}}$, and $y_{j}=\max \left\{C, z_{j}\right\}$ for $j=0,1,2, \ldots$ Then (3.3.9) has the form

$$
y_{j} \leq\left(C p_{j}\right)^{1 / p_{j}} y_{j-1} \quad \text { for } j \in \mathbb{N} .
$$

This can be rewritten as

$$
\log y_{j} \leq C(1+\varrho)^{-j}(1+j)+\log y_{j-1} \quad \text { for } j \in \mathbb{N},
$$

hence the sequence $y_{j}$ is bounded by a constant $C$ independent of $K$. Consequently, there exists $K_{0}$ such that

$$
\left\|\theta_{K}\right\|_{p} \leq K_{0}
$$

independently of $p$ and $K$, which is the desired estimate that enables us to complete the proof of Theorem 3.2.3.

\subsection{Proof of regularity}

We do not know any reference for the claim of Theorem 3.2.4, although it might be possible to obtain the same result using other strategies. The following proof is rather involved but relies essentially on elementary tools (opportune estimates in Hilbert spaces). More specifically, the proof is organized as follows.

- First of all we work, in order, with the domain: first the half space in $\mathbb{R}^{N}$, then the deformed half-space (as given by (3.4.3). Eventually we will transpose the obtained results to the original bounded domain $\Omega$ of Hypothesis 3.2.2 through a localization argument (partition of unity).

- Since we know that we are going to use a localization argument to pass from the unbounded to the bounded domain, we start treating, instead of equation (3.2.1), its (slight) generalization (3.4.1), which contains a linear term more $(A \nabla v+B)$. 
- As already said, we start in the case that $\Omega$ is the half-space in $\mathbb{R}^{N}$. Using an estimation technique which is presented similarly in the book from Nečas [40] for elliptic equations with linear boundary conditions, we prove that a solution to ( 3.4.1) possesses the desired regularity $\nabla v \in L^{2}\left(0, T ; L^{\infty}(\Omega)\right)$. This will be done in five steps: Lemma 3.4.3, 3.4.4, 3.4.5, 3.4.6 and 3.5.3.

We point out that the nonlinear boundary condition $h\left(x, \theta, \theta_{\Gamma}\right)$ necessitates special attention, since the trace of $v$ and of its first and second partial derivatives on the boundary will be involved. The main idea for treating this nonlinear term is to introduce equation (3.4.10), which contains an additional boundary term just in order to manage the trace of $v$ at the boundary. The aim will be to let eventually tend $\delta$ to 0 and translate the results from equation (3.4.10) to equation (3.4.9).

After this, we will dispose of the regularity of the partial derivatives of $v$ sufficient in order to apply an opportune embedding theorem in the set of the anisotropic Sobolev spaces. This indeed requires less for the derivative in the normal direction than for the derivatives in the tangential directions. In this part we will make use of the results contained in the book from Besov, V. P. Il'in, S. M. Nikol'skiı $[6]$.

- Then we consider an $\Omega$ of the form given in (3.4.3), i.e. a "deformed" half-space and also in this case we proof that the solution of 3.4 .1 possesses the desired regularity $\nabla v \in L^{2}\left(0, T ; L^{\infty}(\Omega)\right)$. This is the content of Theorem 3.4.2.

- All the estimates derived in Lemmas 3.4.3-3.4.5 (concerning the partial derivatives of $v$ ) can be transposed to the case of $\Omega$ deformed half-space. This result is contained in Theorem 3.4.1.

- The last step consists in transposing the results to the original bounded domain $\Omega$, by means of a partition of unity argument. This leads to Theorem 3.2.4.

Let us start by considering the equation:

$$
\int_{\Omega}\left(v_{t} \varphi+A(x) \nabla v \cdot \nabla \varphi+B(x, t) \cdot \nabla \varphi-f(x, t) \varphi\right) \mathrm{d} x+\int_{\partial \Omega} h\left(x, v, v_{\Gamma}(x, t)\right) \varphi \mathrm{d} S=0
$$

for every test function $\varphi \in W^{1,2}(\Omega)$, with initial condition $v(x, 0)=v^{0}(x)$. Here $A=\left(A_{i j}\right)_{i, j=1}^{N}: \Omega \rightarrow \mathbb{R}_{\mathrm{sym}}^{N \times N}$ is a symmetric matrix function such that there exists $\kappa>0$ with the property

$$
\forall \xi \in \mathbb{R}^{N}: A(x) \xi \cdot \xi \geq \kappa|\xi|^{2} \quad \text { a. e. }
$$

and $f: Q_{T} \rightarrow \mathbb{R}, B: Q_{T} \rightarrow \mathbb{R}^{N}, B=\left(B_{1}, \ldots, B_{N}\right), h: \partial \Omega \times \mathbb{R}^{2} \rightarrow \mathbb{R}$, and $v_{\Gamma}:$ $\partial \Omega \times(0, T) \rightarrow \mathbb{R}$ are given functions.

The reasons for introducing the functions $A(x)$ and $B(x, t)$, which do not appear in (3.2.1), are purely technical. They arise as a result of deformations of the domain and partition of unity. 
Let us also specify the notations concerning the domain, which will be used in the following proofs.

Consider a set $\Omega \subset \mathbb{R}^{N}$ of the form

$$
\Omega=\left\{\left(x^{\prime}, x_{N}\right): x^{\prime} \in \mathbb{R}^{N-1}, x_{N}>g\left(x^{\prime}\right)\right\},
$$

with a given function $g$. Assume moreover that there exists a ball $\Omega_{k_{0}}$ centered at 0 of radius $k_{0}>0$ such that

$$
B(x, \cdot)=f(x, \cdot)=h(x, \cdot, \cdot)=0 \text { for } x \in \Omega \backslash \Omega_{k_{0}} .
$$

This assumption does not represent a restriction to our aim since we will deal with a localization argument. In particular, it is defined the space:

$$
\Omega=\mathbb{R}_{+}^{N}:=\left\{\left(y^{\prime}, y_{N}\right): y^{\prime} \in \mathbb{R}^{N-1}, y_{N}>0\right\}
$$

We will prove the following regularity results.

Theorem 3.4.1. Let $\Omega$ be as in (3.4.3), and let $g \in W^{2, \infty}\left(\mathbb{R}^{N-1}\right)$. We make the following assumptions:

- $h$ is a globally Lipschitz-continuous function in all variables; furthermore, with $v, v_{\Gamma} \in \mathbb{R}$ fixed, the functions $h\left(\cdot, v, v_{\Gamma}\right), \partial_{\ell} h\left(\cdot, v, v_{\Gamma}\right)$ belong to $L^{2}(\partial \Omega)$ for all $\ell=1, \ldots, N-1$;

- $A \in W^{1, \infty}\left(\Omega ; \mathbb{R}_{\mathrm{sym}}^{N \times N}\right), B \in L^{2}\left(0, T ; W^{1,2}\left(\Omega ; \mathbb{R}^{N}\right)\right), B_{t} \in L^{2}\left(Q_{T} ; \mathbb{R}^{N}\right) ;$

- $v^{0} \in W^{1,2}(\Omega), f \in L^{2}\left(Q_{T}\right), v_{\Gamma} \in L^{2}\left(0, T ; W^{1,2}(\partial \Omega)\right),\left(v_{\Gamma}\right)_{t} \in L^{2}\left(0, T ; L^{2}(\partial \Omega)\right)$.

Let $v \in L^{2}(0, T ; V)$ such that $v_{t} \in L^{2}\left(0, T ; V^{\prime}\right)$ be a solution to (3.4.1).

Then $v$ has the regularity

$$
v_{t} \in L^{2}\left(Q_{T}\right), \quad v \in L^{2}\left(0, T ; W^{2,2}(\Omega)\right), \quad \nabla v \in L^{\infty}\left(0, T ; L^{2}(\Omega)\right) .
$$

Theorem 3.4.2. Let $\Omega$ be as in (3.4.3), and let $g \in W^{3, \infty}\left(\mathbb{R}^{N-1}\right)$. We make the following assumptions:

- $h$ is of class $W^{2, \infty}$ with respect to all variables; furthermore, with $v, v_{\Gamma} \in \mathbb{R}$ fixed, the functions $h\left(\cdot, v, v_{\Gamma}\right), \partial_{\ell} h\left(\cdot, v, v_{\Gamma}\right), \partial_{\ell} \partial_{m} h\left(\cdot, v, v_{\Gamma}\right)$ belong to $L^{2}(\partial \Omega)$ for all $\ell, m=1, \ldots, N-1$;

$$
\text { - } A \in W^{2, \infty}\left(\Omega ; \mathbb{R}_{\mathrm{sym}}^{N \times N}\right), B \in L^{2}\left(0, T ; W^{2,2}\left(\Omega ; \mathbb{R}^{N}\right)\right), B_{t} \in L^{2}\left(Q_{T} ; \mathbb{R}^{N}\right) ;
$$

- $v^{0} \in W^{2,2}(\Omega), f \in L^{2}\left(0, T ; W^{1,2}(\Omega)\right), v_{\Gamma} \in L^{2}\left(0, T ; W^{2,2}(\partial \Omega)\right)$, $\left(v_{\Gamma}\right)_{t} \in L^{2}\left(0, T ; W^{1,2}(\partial \Omega)\right)$.

Let $v \in L^{2}(0, T ; V)$ such that $v_{t} \in L^{2}\left(0, T ; V^{\prime}\right)$ be a solution to (3.4.1). If $N \leq 3$, then $v$ has the regularity

$$
v_{t} \in L^{2}\left(Q_{T}\right), \quad v \in L^{2}\left(0, T ; W^{2,2}(\Omega)\right) \quad \nabla v \in L^{2}(0, T ; C(\bar{\Omega}) .
$$


Still for theorem 3.4.1 we are not aware of any reference. Therefore we proceed step by step, showing how Theorem 3.4.1 and to Theorem 3.4.2 follow from the estimates contained in the lemmas below. Before passing to the lemmas, we recall some classical estimates that are needed both here and afterwards in the proof of uniqueness.

- Young's inequality:

$$
a b \leq \epsilon a^{p}+C_{\epsilon} b^{p^{\prime}} \quad \forall a \geq 0, \quad b \geq 0
$$

- Hölder inequality:

$$
\|f\|_{r} \leq\|f\|_{p}^{\alpha}\|f\|_{q}^{1-\alpha} \frac{1}{r}=\frac{\alpha}{p}+\frac{1-\alpha}{q}, \quad p \leq r \leq q,
$$

for $f \in L^{p} \cap L^{q}, 1 \leq p \leq q \leq \infty$.

- Minkowski inequality:

$$
\left(\int_{Y}\left(\int_{X} f(x, y) d x\right)^{p}\right)^{1 / p} d y \leq \int_{X}\left(\int_{Y} f^{p}(x, y) d y\right)^{1 / p} d x, \quad 1<p<\infty
$$

As mentioned before, we first consider the case of $\Omega$ half-space of the form (3.4.5). For a general function $w \in W^{1,2}\left(\mathbb{R}_{+}^{N}\right)$, we have the identity

$$
w^{2}\left(y^{\prime}, y_{N}\right)-w^{2}\left(y^{\prime}, 0\right)=2 \int_{0}^{y_{N}} w\left(y^{\prime}, z\right) \partial_{N} w\left(y^{\prime}, z\right) \mathrm{d} z
$$

hence, for every $M>0$, integrating w.r.t. $y_{N}$, we have by Fubini Theorem that

$$
w^{2}\left(y^{\prime}, 0\right) \leq \frac{1}{M} \int_{0}^{M} w^{2}\left(y^{\prime}, y_{N}\right) \mathrm{d} y_{N}+2 \int_{0}^{M}\left|w\left(y^{\prime}, z\right)\right|\left|\partial_{N} w\left(y^{\prime}, z\right)\right| \mathrm{d} z .
$$

Letting $M$ tend to $\infty$, we obtain the trace interpolation formula

$$
|w(\cdot, 0)|_{L^{2}\left(\mathbb{R}^{N-1}\right)}^{2} \leq 2|w|_{2}\left|\partial_{N} w\right|_{2}
$$

or, as a consequence,

$$
\forall \varepsilon>0 \quad \exists C_{\varepsilon}>0 \quad \forall w \in W^{1,2}\left(\mathbb{R}_{+}^{N}\right):|w(\cdot, 0)|_{L^{2}\left(\mathbb{R}^{N-1}\right)}^{2} \leq C_{\varepsilon}|w|_{2}^{2}+\varepsilon|\nabla w|_{2}^{2} .
$$

For domains of the form (3.4.3) with a Lipschitz-continuous function $g$, this inequality reads after substitution in the integrals as

$$
\forall \varepsilon>0 \quad \exists C_{\varepsilon}>0 \quad \forall w \in W^{1,2}(\Omega): \quad \int_{\partial \Omega}|w(x)|^{2} \mathrm{~d} S \leq C_{\varepsilon}|w|_{2}^{2}+\varepsilon|\nabla w|_{2}^{2} .
$$

Proceeding for instance as in [40], by a partition of unity argument, we obtain (3.4.8) for every Lipschitz-continuous domain $\Omega$. 
If $\Omega$ is the half-space, i.e. in the context of (3.4.5), we rewrite equations (3.4.1) as

$\int_{\mathbb{R}_{+}^{N}}\left(v_{t} \varphi+(A \nabla v+B) \cdot \nabla \varphi-f \varphi\right) \mathrm{d} y+\int_{\mathbb{R}^{N-1}} h\left(y^{\prime}, v\left(y^{\prime}, 0, t\right), v_{\Gamma}\left(y^{\prime}, t\right)\right) \varphi\left(y^{\prime}, 0\right) \mathrm{d} y^{\prime}=0$.

Ad mentioned before, we introduce a sort of "regularized" problem. Let $\delta \geq 0$ and $\nabla_{y^{\prime}}$ denote the partial gradient $\nabla_{y^{\prime}} v=\left(\partial_{1} v, \ldots, \partial_{N-1} v\right)$. We consider the equation

$$
\begin{aligned}
& \int_{\mathbb{R}_{+}^{N}}\left(v_{t} \varphi+(A \nabla v+B) \cdot \nabla \varphi-f \varphi\right) \mathrm{d} y \\
& \quad+\int_{\mathbb{R}^{N-1}}\left(\delta \nabla_{y^{\prime}} v\left(y^{\prime}, 0, t\right) \cdot \nabla_{y^{\prime}} \varphi\left(y^{\prime}, 0\right)+h\left(y^{\prime}, v\left(y^{\prime}, 0, t\right), v_{\Gamma}\left(y^{\prime}, t\right)\right) \varphi\left(y^{\prime}, 0\right)\right) \mathrm{d} y^{\prime}=0
\end{aligned}
$$

which has to be satisfied in the case $\delta>0$ for every test function $\varphi\left(y^{\prime}, y_{N}\right)$ from the space

$$
W=\left\{\varphi \in W^{1,2}\left(\mathbb{R}_{+}^{N}\right): \varphi(\cdot, 0) \in W^{1,2}\left(\mathbb{R}^{N-1}\right)\right\} .
$$

Our goal will be to derive bounds for its solution independent of $\delta$, which then imply the corresponding estimates for the solution of (3.4.9).

Lemma 3.4.3. Let $v^{0} \in W^{1,2}\left(\mathbb{R}_{+}^{N}\right), f \in L^{2}\left(\mathbb{R}_{+}^{N} \times(0, T)\right), A \in W^{1, \infty}\left(\mathbb{R}_{+}^{N} ; \mathbb{R}_{\mathrm{sym}}^{N \times N}\right)$, $B \in L^{2}\left(0, T ; W^{1,2}\left(\mathbb{R}_{+}^{N} ; \mathbb{R}^{N}\right)\right)$, and $v_{\Gamma} \in L^{2}\left(0, T ; W^{1,2}\left(\mathbb{R}^{N-1}\right)\right)$ be given.

Let exist a function $h_{1} \in L^{2}\left(\mathbb{R}^{N-1}\right) \cap L^{\infty}\left(\mathbb{R}^{N-1}\right)$ such that $h$ together with all its first derivatives is bounded above by $h_{1}$.

Then there exists a constant $C_{1}>0$ independent of $\delta \geq 0$ such that the solution $v$ to (3.4.10) satisfies for all $t \in[0, T]$ the estimate

$$
\left|\partial_{\ell} v(t)\right|_{2}^{2}+\int_{0}^{T}\left|\nabla \partial_{\ell} v(t)\right|_{2}^{2} \mathrm{~d} t+\delta \int_{0}^{T} \int_{\mathbb{R}^{N-1}}\left|\partial_{\ell} \partial_{m} v\left(y^{\prime}, 0, t\right)\right|^{2} \mathrm{~d} y^{\prime} \mathrm{d} t \leq C_{1}
$$

for all $\ell, m=1, \ldots, N-1$.

Proof. A solution $v \in L^{2}(0, T ; W)$ such that $v_{t} \in L^{2}\left(0, T ; W^{\prime}\right)$ to $(3.4 .10)$ can be constructed e.g. as follows. For $k>k_{0}$ (cf. (3.4.4)), we denote by $\mathbb{R}_{k}^{N}$ the intersection of $\mathbb{R}_{+}^{N}$ with the ball $\Omega_{k}$, and by $\Gamma_{k}^{0}, \Gamma_{k}^{1}$ the flat and the curved part of the boundary of $\mathbb{R}_{k}^{N}$, respectively. Instead of (3.4.10), we consider the problem

$$
\begin{aligned}
& \int_{\mathbb{R}_{k}^{N}}\left(v_{t} \varphi+(A \nabla v+B) \cdot \nabla \varphi-f \varphi\right) \mathrm{d} y \\
& \quad+\int_{\Gamma_{k}^{0}}\left(\delta \nabla_{y^{\prime}} v\left(y^{\prime}, 0, t\right) \cdot \nabla_{y^{\prime}} \varphi\left(y^{\prime}, 0\right)+h\left(y^{\prime}, v\left(y^{\prime}, 0, t\right), v_{\Gamma}\left(y^{\prime}, t\right)\right) \varphi\left(y^{\prime}, 0\right)\right) \mathrm{d} y^{\prime}=0,
\end{aligned}
$$

with zero Dirichlet boundary condition on $\bar{\Gamma}_{k}^{1}$. We define the spaces

$$
W_{k}=\left\{\varphi \in W^{1,2}\left(\mathbb{R}_{k}^{N}\right): \varphi(\cdot, 0) \in W^{1,2}\left(\Gamma_{k}^{0}\right), \varphi=0 \text { on } \bar{\Gamma}_{k}^{1}\right\}
$$

Using Galerkin approximations, the compactness lemma in [36, Section 1.5], and the compact embedding of $W_{k}$ in the space $H_{k}=\left\{\varphi \in L^{2}\left(\mathbb{R}_{k}^{N}\right): \varphi(\cdot, 0) \in L^{2}\left(\Gamma_{k}^{0}\right)\right\}$, we 
prove the existence of a solution $v_{k}$ to (3.4.12), which we extend by 0 to $\mathbb{R}_{+}^{N}$. The solution is unique and satisfies the bound

$$
\left|v_{k}(T)\right|_{2}^{2}+\int_{0}^{T}\left|\nabla v_{k}(t)\right|_{2}^{2} \mathrm{~d} t+\delta \int_{0}^{T} \int_{\mathbb{R}^{N-1}}\left|\nabla_{y^{\prime}} v_{k}\left(y^{\prime}, 0, t\right)\right|^{2} \mathrm{~d} y^{\prime} \mathrm{d} t \leq C_{0}
$$

by virtue of (3.4.7), (3.4.4), and Gronwall lemma, with a constant $C_{0}$ independent of $\delta$ and $k$. This, and the fact that the nonlinear term has compact support independent of $k$, enable us to pass to the limit as $k \rightarrow \infty$ and find a solution $v$ to (3.4.10) satisfying the estimate (3.4.13).

To obtain higher order estimates, we use the method of the difference quotients to approximate the weak derivatives of $v$. In this sense it is used for example in [14] in order to improve the regularity of solutions of elliptic partial differential equations.

We denote by $e_{\ell}$ for $\ell=1, \ldots, N$ the $\ell$-th unit coordinate vector, and by $D_{s}^{\ell}$ for $s \neq 0$ the linear mapping

$$
D_{s}^{\ell}(v)(y, t)=\frac{1}{s}\left(v\left(y+s e_{\ell}, t\right)-v(y, t)\right)
$$

We will use the following formula which hold in a generic subspace $U$ of $\mathbb{R}^{N}$.

$$
\int_{U} w D_{s}^{\ell}(v) \mathrm{d} y=-\int_{U} v D_{s}^{-\ell}(w) \mathrm{d} y
$$

and the properties of differences quotients in relation with weak derivatives, which can be found, for instance, in the book from Evans [14, Chap. 5, Sect. 8, Th. 5].

Now let $\varphi \in W$ be given. In (3.4.10), we choose consecutively test functions $\tilde{\varphi}(y)=$ $\varphi(y)$ and $\tilde{\varphi}(y)=\varphi\left(y-s e_{\ell}\right)$ for some $\ell=1, \ldots, N-1$, and subtract the two identities. This yields, after a suitable substitution, that

$$
\begin{gathered}
\int_{\mathbb{R}_{+}^{N}}\left(D_{s}^{\ell} v_{t} \varphi+\left(A\left(y+s e_{\ell}\right) \nabla\left(D_{s}^{\ell} v\right)+\left(D_{s}^{\ell} A\right) \nabla v+D_{s}^{\ell} B\right) \cdot \nabla \varphi+f(y, t) D_{-s}^{\ell} \varphi\right) \mathrm{d} y(3.4 .14) \\
+\int_{\mathbb{R}^{N-1}}\left(\delta \nabla_{y^{\prime}} D_{s}^{\ell} v\left(y^{\prime}, 0, t\right) \cdot \nabla_{y^{\prime}} \varphi\left(y^{\prime}, 0\right)+D_{s}^{\ell}\left(h\left(y^{\prime}, v\left(y^{\prime}, 0, t\right), v_{\Gamma}\left(y^{\prime}, t\right)\right)\right) \varphi\left(y^{\prime}, 0\right)\right) \mathrm{d} y^{\prime} .
\end{gathered}
$$

For $\varphi(y)=D_{s}^{\ell} v(y, t)$, we have in particular the estimate

$$
\begin{aligned}
\frac{1}{2} \frac{\mathrm{d}}{\mathrm{d} t} \mid & \left.D_{s}^{\ell} v(t)\right|_{2} ^{2}+\kappa\left|\nabla\left(D_{s}^{\ell} v(t)\right)\right|_{2}^{2}+\delta \int_{\mathbb{R}^{N-1}}\left|\nabla_{y^{\prime}} D_{s}^{\ell} v\left(y^{\prime}, 0, t\right)\right|^{2} \mathrm{~d} y^{\prime} \\
\leq & |\nabla A|_{\infty}|\nabla v(t)|_{2}\left|\nabla\left(D_{s}^{\ell} v(t)\right)\right|_{2}+\left(|f(t)|_{2}+|\nabla B(t)|_{2}\right)\left|\nabla\left(D_{s}^{\ell} v(t)\right)\right|_{2} \\
\quad & \quad \int_{\mathbb{R}^{N-1}} h_{1}\left(y^{\prime}\right)\left(1+\left|D_{s}^{\ell} v_{\Gamma}\left(y^{\prime}, t\right)\right|+\left|D_{s}^{\ell} v\left(y^{\prime}, 0, t\right)\right|\right)\left|D_{s}^{\ell} v\left(y^{\prime}, 0, t\right)\right| \mathrm{d} y^{\prime} .
\end{aligned}
$$

We can pass to the limit as $s \rightarrow 0$. From (3.4.7) we have

$$
\left|\partial v_{\ell}\right|_{\mathbb{R}^{N-1}} \leq\left. C_{\epsilon}\left|\partial v_{\ell}\right|_{\mathbb{R}^{N-1}}|+\varepsilon| \nabla_{y^{\prime}} \partial v_{\ell}\right|_{\mathbb{R}^{N-1}}
$$


From this estimate, from (3.4.13) and from Gronwall lemma we obtain

$$
\begin{aligned}
& \left|\partial_{\ell} v(t)\right|_{2}^{2}+\int_{0}^{T}\left|\nabla \partial_{\ell} v(t)\right|_{2}^{2} \mathrm{~d} t+\delta \int_{0}^{T} \int_{\mathbb{R}^{N-1}}\left|\nabla_{y^{\prime}} \partial_{\ell} v\left(y^{\prime}, 0, t\right)\right|^{2} \mathrm{~d} y^{\prime} \mathrm{d} t \\
& \leq C\left(1+C_{0}+\left|\nabla v^{0}\right|_{2}^{2}+|\nabla A|_{\infty}^{2}+\int_{0}^{T}\left(|f(t)|_{2}^{2}+|\nabla B(t)|_{2}^{2}\right) \mathrm{d} t+\int_{0}^{T}\left|\nabla_{y^{\prime}} v_{\Gamma}(t)\right|_{2, \mathbb{R}^{N-1}}^{2} \mathrm{~d} t\right)
\end{aligned}
$$

with a constant $C$ independent of $\delta$, which we wanted to prove.

Lemma 3.4.4. Under the hypotheses of Lemma 3.4.3, assume in addition that: - $\left(v_{\Gamma}\right)_{t} \in L^{2}\left(\mathbb{R}^{N-1} \times(0, T)\right)$ and $B_{t} \in L^{2}\left(\mathbb{R}_{+}^{N} \times(0, T) ; \mathbb{R}^{N}\right)$.

Then there exists a constant $C_{2}$ independent of $\delta \geq 0$ such that the solution $v$ to (3.4.10) satisfies for every $t \in[0, T]$ the estimate

$$
|\nabla v(t)|_{2}^{2}+\delta \int_{\mathbb{R}^{N-1}}\left|\nabla_{y^{\prime}} v\left(y^{\prime}, 0, t\right)\right|^{2} \mathrm{~d} y^{\prime}+\int_{0}^{T}\left(|v(t)|_{W^{2,2}\left(\mathbb{R}_{+}^{N}\right)}^{2}+\left|v_{t}(t)\right|_{2}^{2}\right) \mathrm{d} t \leq C_{2} .
$$

Proof. We discretize Eq. (3.4.10) in time, test by the time increment of $v$, and let the time step tend to 0 . In the limit we obtain the identity

$$
\begin{aligned}
\int_{\mathbb{R}_{+}^{N}}\left(\left|v_{t}\right|^{2}-f v_{t}-B_{t} \cdot \nabla v\right) \mathrm{d} y+\frac{\mathrm{d}}{\mathrm{d} t} \int_{\mathbb{R}_{+}^{N}}\left(\frac{1}{2} A(y) \nabla v+B\right) \cdot \nabla v \mathrm{~d} y \\
+\frac{\mathrm{d}}{\mathrm{d} t} \int_{\mathbb{R}^{N-1}}\left(\frac{\delta}{2}\left|\nabla_{y^{\prime}} v\right|^{2}+\hat{h}\left(y^{\prime}, v, v_{\Gamma}\right)\right) \mathrm{d} y^{\prime}=\int_{\mathbb{R}^{N-1}} \partial_{v_{\Gamma}} \hat{h}\left(y^{\prime}, v, v_{\Gamma}\right)\left(v_{\Gamma}\right)_{t} \mathrm{~d} y^{\prime},
\end{aligned}
$$

where

$$
\hat{h}\left(y^{\prime}, v, v_{\Gamma}\right)=\int_{0}^{v} h\left(y^{\prime}, u, v_{\Gamma}\right) \mathrm{d} u .
$$

We have

$$
\hat{h}\left(y^{\prime}, v, v_{\Gamma}\right) \leq \frac{h_{1}}{2} v^{2}+|v|\left(h_{1}\left|v_{\Gamma}\right|+\left|h\left(y^{\prime}, 0,0\right)\right|\right)
$$

and

$$
\left|\partial_{v_{\Gamma}} \hat{h}\left(y^{\prime}, v, v_{\Gamma}\right)\right| \leq h_{1}|v|
$$

Integrating equation (3.4.18) and using the estimates above, we obtain:

$$
\begin{aligned}
\delta \int_{\mathbb{R}^{N-1}} & \left|\nabla_{y^{\prime}} v\left(y^{\prime}, 0, t\right)\right|^{2} \mathrm{~d} y^{\prime}+\int_{0}^{t}\left|v_{t}(\tau)\right|_{2}^{2} \mathrm{~d} \tau+|\nabla v(t)|_{2}^{2} \\
\leq & C\left(1+\left|\nabla v^{0}\right|_{2}^{2}+\int_{0}^{t}|f(\tau)|_{2}^{2} \mathrm{~d} \tau+\int_{\mathbb{R}^{N-1}}\left|v_{\Gamma}\left(y^{\prime}, t\right)\right|^{2} \mathrm{~d} y^{\prime}\right. \\
& \left.+\int_{0}^{t} \int_{\mathbb{R}^{N-1}}\left|\left(v_{\Gamma}\right)_{t}\left(y^{\prime}, t\right)\right|^{2} \mathrm{~d} y^{\prime} \mathrm{d} \tau\right)
\end{aligned}
$$

with a constant $C$ independent of $\delta$ and $t$ as a consequence of (3.4.7) and Gronwall lemma.

At this point we are very close to the desired estimate (3.4.17). Indeed, by Lemma 3.4.3, we have $\nabla \partial_{\ell} v \in L^{2}\left(\mathbb{R}_{+}^{N} \times(0, T)\right)$ for all $\ell=1, \ldots, N-1$. 
In order to finish the proof, we must show that $\partial_{N}^{2} v \in L^{2}\left(\mathbb{R}_{+}^{n}\right)$. To this aim we now choose in Eq. (3.4.10) any test function $\varphi=\varphi_{0} \in L^{2}\left(0, T ; W^{1,2}\left(\mathbb{R}_{+}^{N}\right)\right)$ with a compact support in $\mathbb{R}_{+}^{N}$. We integrate by parts in all terms except for $A_{N N} \partial_{N} v \partial_{N} \varphi$, and obtain an identity of the form

$$
\int_{0}^{T} \int_{\mathbb{R}_{+}^{N}} A_{N N}(y) \partial_{N} v(y, t) \partial_{N} \varphi_{0}(y, t) \mathrm{d} y \mathrm{~d} t=\int_{0}^{T} \int_{\mathbb{R}_{+}^{N}} \Psi(y, t) \varphi_{0}(y, t) \mathrm{d} y \mathrm{~d} t
$$

with a function $\Psi \in L^{2}\left(\mathbb{R}_{+}^{N} \times(0, T)\right)$. Hence,

$$
\partial_{N}\left(A_{N N}(y) \partial_{N} v(y, t)\right) \in L^{2}\left(\mathbb{R}_{+}^{N} \times(0, T)\right) .
$$

Now according to the uniform ellipticity condition (3.4.2), setting $\xi=e_{n}=(0, \ldots, 0,1)$ we have

$$
A_{N N}(y) \geq \kappa>0
$$

for all $y \in \mathbb{R}_{+}^{n}$. Since $A_{N N} \in W^{1, \infty}\left(\mathbb{R}_{+}^{N}\right)$, we obtain that

$$
\partial_{N}^{2} v \in L^{2}\left(\mathbb{R}_{+}^{n}\right) .
$$

Therewith the proof of Lemma 3.4.4 is complete.

Lemmas 3.4.3 and 3.4.4 enable us to rewrite Eq. (3.4.10) in strong form

$$
\begin{aligned}
& v_{t}-\operatorname{div}(A(y) \nabla v+B(y, t))-f(y, t)=0 \quad \text { a. e. in } \mathbb{R}_{+}^{N} \times(0, T) \\
& \sum_{j=1}^{N} A_{N j} \partial_{j} v+B_{N}-\delta \Delta_{y^{\prime}} v+h\left(y^{\prime}, v, v_{\Gamma}\left(y^{\prime}, t\right)\right)=0 \quad \text { a. e. in } \mathbb{R}^{N-1} \times(0, T)
\end{aligned}
$$

where $\Delta_{y^{\prime}}$ is the Laplacian with respect to $y^{\prime}$.

Lemma 3.4.5. Let $N \leq 3$ and $\delta>0$. Under the hypotheses of Lemma 3.4.4, assume in addition that:

$-v^{0} \in W^{2,2}\left(\mathbb{R}_{+}^{N}\right), f \in L^{2}\left(0, T ; W^{1,2}\left(\mathbb{R}_{+}^{N}\right)\right)$,

$-A \in W^{2, \infty}\left(\mathbb{R}_{+}^{N} ; \mathbb{R}_{\mathrm{sym}}^{N \times N}\right), B \in L^{2}\left(0, T ; W^{2,2}\left(\mathbb{R}_{+}^{N} ; \mathbb{R}^{N}\right)\right)$,

$-v_{\Gamma} \in L^{2}\left(0, T ; W^{2,2}\left(\mathbb{R}^{N-1}\right)\right),\left(v_{\Gamma}\right)_{t} \in L^{2}\left(0, T ; W^{1,2}\left(\mathbb{R}^{N-1}\right)\right)$,

- there exists a function $h_{2} \in L^{2}\left(\mathbb{R}^{N-1}\right) \cap L^{\infty}\left(\mathbb{R}^{N-1}\right)$ such that $h$ together with all its first and second derivatives is bounded above by $h_{2}$.

Then there exists a constant $C_{3}>0$ independent of $\delta$ such that the solution $v$ to (3.4.10) satisfies for all $t \in 0, T]$ the estimate

$$
\left|\partial_{m} \partial_{\ell} v(t)\right|_{2}^{2}+\int_{0}^{T}\left|\nabla \partial_{m} \partial_{\ell} v(t)\right|_{2}^{2} \mathrm{~d} t+\delta \int_{0}^{T} \int_{\mathbb{R}^{N-1}}\left|\partial_{\ell} \partial_{m} \partial_{k} v\left(y^{\prime}, 0, t\right)\right|^{2} \mathrm{~d} y^{\prime} \mathrm{d} t \leq C_{3}
$$

for all $\ell, m, k=1, \ldots, N-1$. 
Proof. Passing to the limit in (3.4.14) as $s \rightarrow 0$, we obtain

$$
\begin{aligned}
& \int_{\mathbb{R}_{+}^{N}}\left(\partial_{\ell} v_{t} \varphi+\left(A \nabla \partial_{\ell} v+\left(\partial_{\ell} A\right) \nabla v+\partial_{\ell} B\right) \cdot \nabla \varphi-\partial_{\ell} f(y, t) \varphi\right) \mathrm{d} y \\
& \quad+\int_{\mathbb{R}^{N-1}}\left(\delta \nabla_{y^{\prime}} \partial_{\ell} v \cdot \nabla_{y^{\prime}} \varphi+\partial_{\ell}\left(h\left(y^{\prime}, v\left(y^{\prime}, 0, t\right), v_{\Gamma}\left(y^{\prime}, t\right)\right)\right) \varphi\left(y^{\prime}, 0\right) \mathrm{d} y^{\prime}=0 .\right.
\end{aligned}
$$

We proceed as in (3.4.14), applying to (3.4.24) the operator $D_{s}^{m}$ with $m \in\{1, \ldots, N-$ $1\}$, and set $\varphi(y)=D_{s}^{m} \partial_{\ell} v(y, t)$, with the intention to proceed as in the proof of Lemma 3.4.3. Here, the situation is more delicate because the second derivatives of the nonlinear term $h\left(y^{\prime}, v, v_{\Gamma}\right)$ will be involved. We obtain indeed the inequality

$$
\begin{gathered}
\frac{1}{2} \frac{\mathrm{d}}{\mathrm{d} t}\left|D_{s}^{m} \partial_{\ell} v(t)\right|_{2}^{2}+\kappa\left|\nabla\left(D_{s}^{m} \partial_{\ell} v(t)\right)\right|_{2}^{2}+\delta \int_{\mathbb{R}^{N-1}}\left|\nabla_{y^{\prime}} D_{s}^{m} \partial_{\ell} v\left(y^{\prime}, 0, t\right)\right|^{2} \mathrm{~d} y^{\prime} \\
\leq \gamma(t)+C \int_{\mathbb{R}^{N-1}}\left(\left|\partial_{\ell} v\right|\left|\partial_{m} v\right|+\left|\partial_{\ell} \partial_{m} v\right|\right)\left|\partial_{\ell} \partial_{m} v\right|\left(y^{\prime}, 0, t\right) \mathrm{d} y^{\prime} \\
\leq \gamma(t)+\tilde{C} \int_{\mathbb{R}^{N-1}}\left(\left|\partial_{\ell} v\right|^{4}+\left|\partial_{\ell} \partial_{m} v\right|^{2}\right)\left(y^{\prime}, 0, t\right) \mathrm{d} y^{\prime}
\end{gathered}
$$

where $\gamma \in L^{1}(0, T)$ includes all terms that have already been estimated above, and $\tilde{C}$ is a constant independent of $t$ and $\delta$. The right hand side of $(3.4 .25)$ is in $L^{1}(0, T)$ by virtue of Lemmas 3.4.3 and 3.4.4 and of the interpolation inequality:

$$
|\psi|_{L^{4}\left(\mathbb{R}^{N-1}\right)} \leq C\left(|\psi|_{L^{2}\left(\mathbb{R}^{N-1}\right)}+|\psi|_{L^{2}\left(\mathbb{R}^{N-1}\right)}^{1 / 2}\left|\nabla_{y^{\prime}} \psi\right|_{L^{2}\left(\mathbb{R}^{N-1}\right)}^{1 / 2}\right)
$$

which holds, for $N=3$, for every $\psi \in W^{1,2}\left(\mathbb{R}^{N-1}\right.$ ) (see [8]), that we apply as follows:

$$
\left|\partial_{\ell} v\right|_{L^{4}\left(\mathbb{R}^{N-1}\right)} \leq C\left(\left|\partial_{\ell} v\right|_{L^{2}\left(\mathbb{R}^{N-1}\right)}+\left|\partial_{\ell} v\right|_{L^{2}\left(\mathbb{R}^{N-1}\right)}^{1 / 2}\left|\nabla_{y^{\prime}} \partial_{\ell} v\right|_{L^{2}\left(\mathbb{R}^{N-1}\right)}^{1 / 2}\right) .
$$

Summarizing we can see that the term

$$
\tilde{C} \int_{\mathbb{R}^{N-1}}\left(\left|\partial_{\ell} v\right|^{4}+\left|\partial_{\ell} \partial_{m} v\right|^{2}\right)\left(y^{\prime}, 0, t\right) \mathrm{d} y^{\prime}
$$

is well defined but can be estimated by a constant depending on $\delta$, since both $\left|\nabla_{y^{\prime}} \partial_{\ell} v\right|_{2}$ and $\left.\left|\partial_{\ell} \partial_{m} v\right|^{2}\right)\left(y^{\prime}, 0, t\right) \mathrm{d} y^{\prime}$, can be estimated in $L^{2}$, through (3.4.11), only by a constant depending on $\delta$. This dependence will be removed later. Thanks to [8][Th.3, Chap.5, Sec.8] we can pass to the limit in (3.4.25) as $s \rightarrow 0$ and use (3.4.26) to end up with

$$
\begin{aligned}
& \frac{1}{2} \frac{\mathrm{d}}{\mathrm{d} t}\left|\partial_{m} \partial_{\ell} v(t)\right|_{2}^{2}+\kappa\left|\nabla \partial_{m} \partial_{\ell} v(t)\right|_{2}^{2}+\delta \int_{\mathbb{R}^{N-1}}\left|\nabla_{y^{\prime}} \partial_{m} \partial_{\ell} v\left(y^{\prime}, 0, t\right)\right|^{2} \mathrm{~d} y^{\prime} \\
& \leq \gamma(t)+C \int_{\mathbb{R}^{N-1}}\left(\left|\nabla_{y^{\prime}} v\right|^{4}+\left|\partial_{\ell} \partial_{m} v\right|^{2}\right)\left(y^{\prime}, 0, t\right) \mathrm{d} y^{\prime} \\
& \leq \gamma(t)+C \int_{\mathbb{R}^{N-1}}\left|\partial_{\ell} \partial_{m} v\right|^{2}\left(y^{\prime}, 0, t\right) \mathrm{d} y^{\prime} \\
& +C\left(\left|\nabla_{y^{\prime}} v(\cdot, 0, t)\right|_{L^{2}\left(\mathbb{R}^{N-1}\right)}^{4}+\left|\nabla_{y^{\prime}} v(\cdot, 0, t)\right|_{L^{2}\left(\mathbb{R}^{N-1)}\right.}^{2}\left|\Delta_{y^{\prime}} v(\cdot, 0, t)\right|_{L^{2}\left(\mathbb{R}^{N-1}\right)}^{2}\right)
\end{aligned}
$$


with a possibly different function $\gamma \in L^{1}(0, T)$ and different constants $C$ independent of $t$ and $\delta$. To continue the estimate of the right hand side of (3.4.27), we need formula (3.4.6), that we recall here:

$$
|w(\cdot, 0)|_{L^{2}\left(\mathbb{R}^{N-1}\right)}^{2} \leq 2|w|_{2}\left|\partial_{N} w\right|_{2}
$$

With this we obtain the following upper bound:

$\gamma(t)+C\left(\left|\partial_{\ell} \partial_{m} v\right|_{2}\left|\partial_{N} \partial_{\ell} \partial_{m} v\right|_{2}+\left|\nabla_{y^{\prime}} v\right|_{2}^{2}\left|\partial_{N} \nabla_{y^{\prime}} v\right|_{2}^{2}+\left|\nabla_{y^{\prime}} v\right|_{2}\left|\partial_{N} \nabla_{y^{\prime}} v\right|_{2}\left|\Delta_{y^{\prime}} v\right|_{2}\left|\partial_{N} \Delta_{y^{\prime}} v\right|_{2}\right)$.

Now we observe that, by Lemma 3.4.3, $\left|\nabla_{y^{\prime}} v\right|_{2} \leq C_{1}$, and $\beta(t):=\left|\partial_{N} \nabla_{y^{\prime}} v(t)\right|_{2}$ belongs to $L^{2}(0, T)$. Hence, by Young's inequality, we obtain from (3.4.27) the estimate

$$
\begin{aligned}
\frac{\mathrm{d}}{\mathrm{d} t} \sum_{\ell, m=1}^{N-1}\left|\partial_{m} \partial_{\ell} v(t)\right|_{2}^{2}+\sum_{\ell, m=1}^{N-1}\left|\nabla \partial_{m} \partial_{\ell} v(t)\right|_{2}^{2} & \\
& \leq \gamma(t)+C \sum_{\ell, m=1}^{2}\left(\left|\partial_{\ell} \partial_{m} v\right|_{2}^{2}+\beta^{2}(t)\left|\Delta_{y^{\prime}} v\right|_{2}^{2}\right),
\end{aligned}
$$

and from Gronwall's argument we obtain (3.4.23).

We now let $\delta$ tend to 0 and prove the following step.

Lemma 3.4.6. Under the hypotheses of Lemma 3.4.5, there exists a constant $C_{4}>0$ such that the solution $v$ to (3.4.9) satisfies for all $t \in[0, T]$ the estimate

$$
\left|\partial_{N} \partial_{\ell} v(t)\right|_{2}^{2}+\int_{0}^{T}\left|\nabla \partial_{N} \partial_{\ell} v(t)\right|_{2}^{2} \mathrm{~d} t \leq C_{4}
$$

for all $\ell=1, \ldots, N-1$.

Proof. From Lemma 3.4.5 it follows that the solution $v$ to (3.4.9) satisfies (3.4.21)(3.4.23) with $\delta=0$. Let us consider now test functions $\varphi$ with compact support in $\mathbb{R}_{+}^{N}$, and apply the operator $D_{s}^{N}$ to Eq. (3.4.9). As a counterpart of (3.4.14), we obtain

$$
\int_{\mathbb{R}_{+}^{N}}\left(D_{s}^{N} v_{t} \varphi+\left(A\left(y+s e_{N}\right) \nabla\left(D_{s}^{N} v\right)+\left(D_{s}^{N} A\right) \nabla v+D_{s}^{N} B\right) \cdot \nabla \varphi-D_{s}^{N} f(y, t) \varphi\right) \mathrm{d} y=0 .
$$

Passing to the limit as $s \rightarrow 0$ yields

$$
\int_{\mathbb{R}_{+}^{N}}\left(\partial_{N} v_{t} \varphi+\left(A(y) \nabla \partial_{N} v+\left(\partial_{N} A(y)\right) \nabla v+\partial_{N} B(y, t)\right) \cdot \nabla \varphi-\partial_{N} f(y, t) \varphi\right) \mathrm{d} y=0 .
$$

Let $V_{0}$ denote the space $W_{0}^{1,2}\left(\mathbb{R}_{+}^{N}\right)$. We choose any $\varphi_{0} \in V_{0}$ and set $\varphi=A_{N \ell} \varphi_{0}$ in (3.4.24) and obtain

$$
\begin{aligned}
& \int_{\mathbb{R}_{+}^{N}}\left(\partial_{\ell} v_{t} A_{N \ell} \varphi_{0}+\left(A \nabla \partial_{\ell} v+\left(\partial_{\ell} A\right) \nabla v+\partial_{\ell} B\right) \cdot \nabla A_{N \ell} \varphi_{0}\right. \\
& \left.-\partial_{\ell} f(y, t) A_{N \ell} \varphi_{0}\right) \mathrm{d} y=0 .
\end{aligned}
$$


Analogously we choose $\varphi=A_{N N} \varphi_{0}$ and set it in (3.4.31)

$$
\begin{gathered}
\int_{\mathbb{R}_{+}^{N}}\left(\partial_{N} v_{t} A_{N N} \varphi_{0}+\left(A(y) \nabla \partial_{N} v+\left(\partial_{N} A(y)\right) \nabla v+\partial_{N} B(y, t)\right) \cdot \nabla A_{N N} \varphi_{0}\right. \\
\left.\left.\quad-\partial_{N} f(y, t)\right) A_{N N} \varphi_{0}\right) \mathrm{d} y=0 .
\end{gathered}
$$

To proceed with the computations, we need the formula

$A \nabla \partial_{\ell} v \cdot \nabla\left(A_{N \ell} \varphi_{0}\right)=A \nabla\left(A_{N \ell} \partial_{\ell} v\right) \cdot \nabla \varphi_{0}+\left(A \nabla \partial_{\ell} v \cdot \nabla A_{N \ell}\right) \varphi_{0}-\partial_{\ell} v\left(A \nabla A_{N \ell} \cdot \nabla \varphi_{0}\right)$.

Now, with a computation, we can rewrite the equations (3.4.32),(3.4.33) as

$$
\begin{gathered}
\int_{\mathbb{R}_{+}^{N}}(A_{N \ell} \partial_{\ell} v_{t} \varphi_{0}+A \nabla\left(A_{N \ell} \partial_{\ell} v\right) \cdot \nabla \varphi_{0}+\underbrace{\left(A \nabla \partial_{\ell} v \cdot \nabla A_{N \ell}\right) \varphi_{0}}_{I}-\underbrace{\partial_{\ell} v\left(A \nabla A_{N \ell} \cdot \nabla \varphi_{0}\right)}_{I I} \\
+\underbrace{\left(\partial_{\ell} A \nabla v+\partial_{\ell} B\right) \cdot \nabla\left(A_{N \ell} \varphi_{0}\right)}_{I I I}-A_{N \ell} \partial_{\ell} f(y, t) \varphi_{0}) \mathrm{d} y=0
\end{gathered}
$$

for all $\ell=1, \ldots, N$.

Consider now the function

$$
w=\sum_{\ell=1}^{N} A_{N \ell} \partial_{\ell} v
$$

Summing up the above identities over $\ell$ and as a consequence of (3.4.22) with $\delta=0$, we can see that $w$ is a solution of the inhomogeneous Dirichlet problem

$$
\int_{\mathbb{R}_{+}^{N}}\left(w_{t} \varphi_{0}+A(y) \nabla w \cdot \nabla \varphi_{0}-f_{1}(y, t) \varphi_{0}\right) \mathrm{d} y=0 \quad \forall \varphi_{0} \in V_{0},
$$

with boundary condition

$$
w\left(y^{\prime}, 0, t\right)+B_{N}\left(y^{\prime}, 0, t\right)+h\left(y^{\prime}, v\left(y^{\prime}, 0, t\right), v_{\Gamma}\left(y^{\prime}, t\right)\right)=0
$$

on $\mathbb{R}^{N-1} \times(0, T)$. The function $f_{1}$ in $(3.4 .35)$ is given by:

$$
f_{1}=\sum_{\ell=1}^{N}(A_{N \ell} \partial_{\ell} f-\underbrace{A \nabla \partial_{\ell} v \cdot \nabla A_{N \ell}}_{I}-\underbrace{\operatorname{div}\left(\left(\partial_{\ell} v\right) A \nabla A_{N \ell}\right)}_{I I}+\underbrace{A_{N \ell} \operatorname{div}\left(\left(\partial_{\ell} A\right) \nabla v+\partial_{\ell} B\right)}_{I I I}),
$$

Hence $f_{1}$ belongs to $L^{2}\left(\mathbb{R}_{+}^{N} \times(0, T)\right)$. We now fix a smooth function $\varrho$ with compact support in $\mathbb{R}_{+}$and such that $\varrho(0)=1$, and set

$$
w_{1}(y, t)=B_{N}(y, t)+\varrho\left(y_{N}\right) h\left(y^{\prime}, v(y, t), v_{\Gamma}\left(y^{\prime}, t\right)\right) .
$$

$w_{1}$ so defined can be seen as a prolongation of the boundary condition to $\mathbb{R}_{+}^{N} \times(0, T)$. The function $w_{0}:=w-w_{1}$ is a solution to the homogeneous Dirichlet problem for the following counterpart of (3.4.35)

$$
\int_{\mathbb{R}_{+}^{N}}\left(\left(w_{0}\right)_{t} \varphi_{0}+A(y) \nabla w_{0} \cdot \nabla \varphi_{0}-f_{2}(y, t) \varphi_{0}\right) \mathrm{d} y=0 \quad \forall \varphi_{0} \in V_{0}
$$


where

$$
f_{2}=f_{1}-\left(w_{1}\right)_{t}+\operatorname{div} A(y) \nabla w_{1} .
$$

Let us check that $f_{2} \in L^{2}\left(\mathbb{R}_{+}^{N} \times(0, T)\right)$. By virtue of Lemmas 3.4.4-3.4.5, the delicate term in $f_{2}$ is $\operatorname{div} A(y) \nabla w_{1}$, since it involves, due to the nonlinearity of $h$ terms of the form $\partial_{\ell}^{2} v$. These terms will be bounded in $L^{2}$ provided we prove that

$$
\nabla v \in L^{4}\left(\mathbb{R}_{+}^{N} \times(0, T)\right) .
$$

To this end, we refer to [6, Vol I, Theorem 10.2], see also Remark 3, which states that there exists a constant $C>0$ such that for every function $\xi \in L^{2}\left(\mathbb{R}_{+}^{N} \times(0, T)\right)$ with the regularity $\partial_{\ell}^{2} \xi, \partial_{N} \xi \in L^{2}\left(\mathbb{R}_{+}^{N} \times(0, T)\right)$ for all $\ell=1, \ldots, N-1$, and for every $\sigma \in(0,1]$ we have the inequality (note that $N \leq 3$ !)

$$
|\xi|_{4} \leq C\left(\sigma^{-1 / 2}|\xi|_{2}+\sigma^{1 / 2}\left(\left|\partial_{N} \xi\right|_{2}+\sum_{\ell=1}^{N-1}\left|\partial_{\ell}^{2} \xi\right|_{2}\right)\right) .
$$

This can be equivalently written as

$$
|\xi|_{4} \leq C\left(|\xi|_{2}+|\xi|_{2}^{1 / 2}\left(\left|\partial_{N} \xi\right|_{2}+\sum_{\ell=1}^{N-1}\left|\partial_{\ell}^{2} \xi\right|_{2}\right)^{1 / 2}\right)
$$

In (3.4.43), we choose $\xi=\partial_{k} v(t)$ for $k=1, \ldots, N$ and a. e. $t$. From Lemmas 3.4.43.4 .5 we obtain $(3.4 .41)$, hence $f_{2} \in L^{2}\left(\mathbb{R}_{+}^{N} \times(0, T)\right)$.

Following the same idea as in the proof of Lemma 3.4.3, we now apply the operator $D_{s}^{\ell}$ to Eq. (3.4.39) for $\ell=1, \ldots, N-1$ and test by $\varphi_{0}=D_{s}^{\ell} w_{0}$.

Using the identity $\int\left(D_{s}^{\ell} w_{1}\right)_{t} D_{s}^{\ell} w_{0} \mathrm{~d} y=\int\left(w_{1}\right)_{t} D_{-s}^{\ell} D_{s}^{\ell} w_{0} \mathrm{~d} y$, we may let $s$ tend to 0 and conclude that

$$
\partial_{\ell} \nabla w_{0} \in L^{2}\left(\mathbb{R}_{+}^{N} \times(0, T)\right)
$$

for all $\ell=1, \ldots, N-1$. By Lemma 3.4.5, and since $A_{N N} \geq \kappa$, we obtain that

$$
\partial_{\ell} \partial_{N}^{2} v \in L^{2}\left(\mathbb{R}_{+}^{N} \times(0, T)\right)
$$

for all $\ell=1, \ldots, N-1$, and the proof is complete.

\subsection{An anisotropic embedding theorem}

Here we prove here an embedding theorem for anisotropic Sobolev spaces that is needed for the proof of Theorem 3.4.1. For a vector $\mathbf{p}=\left(p_{1}, \ldots, p_{N}\right), 1 \leq p_{i}<\infty$, we define the space $L^{\mathbf{P}}\left(\mathbb{R}^{N}\right)$ as the subspace of $L^{1}\left(\mathbb{R}^{N}\right)$ of functions $u$ such that the norm

$$
\|u\|_{\mathbf{p}}=\left(\int_{\mathbb{R}}\left(\cdots \int_{\mathbb{R}}\left(\int_{\mathbb{R}}|u(x)|^{p_{1}} \mathrm{~d} x_{1}\right)^{p_{2} / p_{1}} \mathrm{~d} x_{2} \ldots\right)^{p_{N} / p_{N-1}} \mathrm{~d} x_{N}\right)^{1 / p_{N}}
$$


is finite. For a matrix $\mathbf{P}=\left(P_{i j}\right)_{i, j=1}^{N}, P_{i j}=1 / p_{i j}, 1 \leq p_{i j}<\infty$, we define the anisotropic Sobolev space

$$
W^{1, \mathbf{P}}\left(\mathbb{R}^{N}\right)=\left\{u \in L^{1}\left(\mathbb{R}^{N}\right): \frac{\partial u}{\partial x_{i}} \in L^{\mathbf{p}_{i}}\left(\mathbb{R}^{N}\right), i=1, \ldots, N\right\}
$$

where $\mathbf{p}_{i}=\left(p_{i 1}, \ldots, p_{i N}\right)$.

We denote by $\mathbf{I}$ the identity $N \times N$ matrix, and by $\mathbf{1}$ the vector $\mathbf{1}=(1,1, \ldots, 1)$. The spectral radius $\varrho(\mathbf{P})$ of $\mathbf{P}$ is defined as

$$
\varrho(\mathbf{P})=\max \{|\lambda|: \lambda \in \mathbb{C}, \operatorname{det}(\mathbf{P}-\lambda \mathbf{I})=0\}=\limsup _{n \rightarrow \infty}\left|\mathbf{P}^{n}\right|^{1 / n} .
$$

Theorem 3.5.1. Let $\varrho(\mathbf{P})<1$, and let

$$
(\mathbf{I}-\mathbf{P})^{-1} \mathbf{1}=\mathbf{b}=\left(b_{1}, \ldots, b_{N}\right) .
$$

Then $W^{1, \mathbf{P}}\left(\mathbb{R}^{N}\right)$ is embedded in $L^{\infty}\left(\mathbb{R}^{N}\right)$, and there exists a constant $C>0$ such that each $u \in W^{1, \mathbf{P}}\left(\mathbb{R}^{N}\right)$ has for all $x, z \in \mathbb{R}^{N}$ the Hölder property

$$
|u(z)-u(x)| \leq C\|u\|_{W^{1, \mathbf{P}\left(\mathbb{R}^{N}\right)}} \sum_{i=1}^{N}\left|z_{i}-x_{i}\right|^{1 / b_{i}} .
$$

The identity (3.5.4) can be written as

$$
\mathbf{b}=\left(\mathbf{I}+\mathbf{P}+\mathbf{P}^{2}+\ldots\right) \mathbf{1} .
$$

Since all entries of $\mathbf{P}$ are positive, we obtain $b_{i}>1$ for all $i$, so that the right hand side of (3.5.5) is meaningful. Note also that in the isotropic case $p_{i j}=p$, Theorem 3.5.1 gives the well-known embedding condition $p>N$ with Hölder exponent $1 / b=1-(N / p)$.

Proof. Following [6], we fix a smooth function $\Phi$ with compact support in $\mathbb{R}^{N}$ such that $\int_{\mathbb{R}^{N}} \Phi(x) \mathrm{d} x=1$, and for $\sigma>0$ and $u \in W^{1, \mathbf{P}}\left(\mathbb{R}^{N}\right)$ set

$$
u^{\sigma}(x)=\sigma^{-|\mathbf{b}|} \int_{\mathbb{R}^{N}} \Phi\left(\frac{x-y}{\sigma^{\mathbf{b}}}\right) u(y) \mathrm{d} y,
$$

where $|\mathbf{b}|=\sum_{i=1}^{N} b_{i}$ and

$$
\frac{x-y}{\sigma^{\mathbf{b}}}=\left(\frac{x_{1}-y_{1}}{\sigma^{b_{1}}}, \ldots, \frac{x_{N}-y_{N}}{\sigma^{b_{N}}}\right) .
$$

By substitution, we have the identity

$$
u^{\sigma}(x)=\int_{\mathbb{R}^{N}} \Phi(z) u\left(x-\sigma^{\mathbf{b}} z\right) \mathrm{d} z,
$$

which implies that

$$
\lim _{\sigma \rightarrow 0}\left|u^{\sigma}-u\right|_{1}=0
$$


We differentiate $u^{\sigma}$ with respect to $\sigma$, integrate by parts with respect to $y$, and obtain

$$
\frac{\partial u^{\sigma}(x)}{\partial \sigma}=-\sum_{i=1}^{N} \sigma^{-|\mathbf{b}|-1+b_{i}} \int_{\mathbb{R}^{N}} \Psi_{i}\left(\frac{x-y}{\sigma^{\mathbf{b}}}\right) \frac{\partial u(y)}{\partial y_{i}} \mathrm{~d} y,
$$

where

$$
\Psi_{i}(z)=b_{i} z_{i} \Phi(z) \quad \text { for } z \in \mathbb{R}^{N} .
$$

By the anisotropic Hölder inequality we have

$$
\left|\frac{\partial u^{\sigma}(x)}{\partial \sigma}\right| \leq \sum_{i=1}^{N} \sigma^{-|\mathbf{b}|-1+b_{i}}\left\|\Psi_{i}\left(\frac{\cdot}{\sigma^{\mathbf{b}}}\right)\right\|_{\mathbf{p}_{i}^{\prime}}\left\|\frac{\partial u}{\partial y_{i}}\right\|_{\mathbf{p}_{i}}
$$

where $\mathbf{p}_{i}^{\prime}$ is the componentwise conjugate of $\mathbf{p}_{i}$. By substitution, we have

$$
\left\|\Psi_{i}\left(\frac{\cdot}{\sigma^{\mathbf{b}}}\right)\right\|_{\mathbf{p}_{i}^{\prime}}=\sigma^{\sum_{j=1}^{N} b_{j} / p_{i j}^{\prime}}\left\|\Psi_{i}\right\|_{\mathbf{p}_{i}^{\prime}}=\sigma^{|\mathbf{b}|-(\mathbf{P b})_{i}}\left\|\Psi_{i}\right\|_{\mathbf{p}_{i}^{\prime}} .
$$

This and (3.5.4) yield the following estimate independent of $\sigma$ and $x$ :

$$
\left|\frac{\partial u^{\sigma}(x)}{\partial \sigma}\right| \leq \sum_{i=1}^{N} \sigma^{b_{i}-(\mathbf{P b})_{i}-1}\left\|\Psi_{i}\right\|_{\mathbf{p}_{i}^{\prime}}\left\|\frac{\partial u}{\partial y_{i}}\right\|_{\mathbf{p}_{i}}=\sum_{i=1}^{N}\left\|\Psi_{i}\right\|_{\mathbf{p}_{i}^{\prime}}\left\|\frac{\partial u}{\partial y_{i}}\right\|_{\mathbf{p}_{i}}=: U
$$

For $\sigma>\tilde{\sigma}>0$ we have

$$
\left|u^{\sigma}(x)-u^{\tilde{\sigma}}(x)\right| \leq(\sigma-\tilde{\sigma}) U
$$

hence $u^{\sigma}$ converges uniformly in $L^{\infty}\left(\mathbb{R}^{N}\right)$ as $\sigma \rightarrow 0$. In view of (3.5.8), its limit is $u$, which thus belongs to $L^{\infty}\left(\mathbb{R}^{N}\right) \cap C\left(\mathbb{R}^{N}\right)$, and we have for all $\sigma>0$ the embedding inequality

$$
|u(x)| \leq\left|u^{\sigma}(x)\right|+\sigma U \leq|\Phi|_{\infty} \sigma^{-\mid \mathbf{b}}|u|_{1}+\sigma U .
$$

To prove the Hölder estimate, we replace $u(x)$ in (3.5.13) by $u\left(x+h e_{i}\right)-u(x)$, where $e_{i}$ is the $i$-th unit coordinate vector and $h>0$ is arbitrary. We obtain

$$
\left|u\left(x+h e_{i}\right)-u(x)\right| \leq\left|u^{\sigma}\left(x+h e_{i}\right)-u^{\sigma}(x)\right|+2 \sigma U,
$$

where

$$
\begin{aligned}
u^{\sigma}\left(x+h e_{i}\right)-u^{\sigma}(x) & =\sigma^{-|\mathbf{b}|} \int_{\mathbb{R}^{N}} \Phi\left(\frac{x-y}{\sigma^{\mathbf{b}}}\right)\left(u\left(y+h e_{i}\right)-u(y)\right) \mathrm{d} y \\
& =-\sigma^{-|\mathbf{b}|} \int_{0}^{h} \int_{\mathbb{R}^{N}} \Phi\left(\frac{x-y}{\sigma^{\mathbf{b}}}\right) \frac{\partial u}{\partial y_{i}}\left(y+s e_{j}\right) \mathrm{d} y \mathrm{~d} s
\end{aligned}
$$

This and (3.5.11) entail

$$
\left|u^{\sigma}\left(x+h e_{i}\right)-u^{\sigma}(x)\right| \leq h \sigma^{-|\mathbf{b}|}\left\|\Psi_{i}\left(\frac{\cdot}{\sigma^{\mathbf{b}}}\right)\right\|_{\mathbf{p}_{i}^{\prime}}\left\|\frac{\partial u}{\partial y_{i}}\right\|_{\mathbf{p}_{i}} \leq h \sigma^{-(\mathbf{P b})_{i}}\left\|\Psi_{i}\right\|_{\mathbf{p}_{i}^{\prime}}\left\|\frac{\partial u}{\partial y_{i}}\right\|_{\mathbf{p}_{i}} .
$$


We thus conclude from (3.5.14) that there exists a constant $C>0$ such that for all $u \in W^{1, \mathbf{P}}\left(\mathbb{R}^{N}\right), x \in \mathbb{R}^{N}, \sigma>0$, and $h>0$ we have

$$
\left|u\left(x+h e_{i}\right)-u(x)\right| \leq C\left(h \sigma^{-(\mathbf{P b})_{i}}+\sigma\right) \sum_{j=1}^{N}\left\|\frac{\partial u}{\partial y_{j}}\right\|_{\mathbf{p}_{j}} .
$$

In particular, for $\sigma=h^{1 / b_{i}}$ we obtain, by virtue of (3.5.4), the formula

$$
\left|u\left(x+h e_{i}\right)-u(x)\right| \leq C h^{1 / b_{i}}\|u\|_{W^{1, \mathbf{P}\left(\mathbb{R}^{N}\right)}},
$$

and (3.5.5) follows from the triangle inequality.

Corollary 3.5.2. The space:

$$
Y:=\left\{w \in L^{1}\left(\mathbb{R}_{+}^{N}\right): \partial_{N} w \in X^{p_{0}, q_{0}}, \quad \nabla_{y^{\prime}} w \in X^{p_{1}, q_{1}}\right\}
$$

satisfies the condition in Theorem 3.5.1 if and only if

$$
\frac{p_{0}^{\prime}}{p_{1} q_{0}}+\frac{1}{q_{1}}<\frac{1}{N-1}
$$

Proof. The matrix $\mathbf{P}-\lambda \mathbf{I}$ has the form

$$
\mathbf{P}-\lambda \mathbf{I}=\left(\begin{array}{ccccc}
1 / q_{1}-\lambda & 1 / q_{1} & \ldots & 1 / q_{1} & 1 / p_{1} \\
1 / q_{1} & 1 / q_{1}-\lambda & \ldots & 1 / q_{1} & 1 / p_{1} \\
& & \ldots & & \\
1 / q_{1} & 1 / q_{1} & \ldots & 1 / q_{1}-\lambda & 1 / p_{1} \\
1 / q_{0} & 1 / q_{0} & \ldots & 1 / q_{0} & 1 / p_{0}-\lambda
\end{array}\right),
$$

and its determinant is

$$
\operatorname{det}(\mathbf{P}-\lambda \mathbf{I})=(-\lambda)^{N-2}\left(\left(\frac{N-1}{q_{1}}-\lambda\right)\left(\frac{1}{p_{0}}-\lambda\right)-\frac{N-1}{q_{0} p_{1}}\right) .
$$

We easily check that all roots of the equation $\operatorname{det}(\mathbf{P}-\lambda \mathbf{I})=0$ are in absolute value smaller that 1 if and only if condition (3.5.20) holds.

Remark 3. The embedding formula (3.4.42) in $\mathbb{R}^{3}$ can be derived from (3.5.6), where we set $b_{1}=b_{2}=\frac{1}{2}, b_{3}=1$. Put $u\left(y^{\prime}, y_{N}\right)=\xi\left(y^{\prime}, y_{N}\right)$ for $y_{N}>0, u\left(y^{\prime}, y_{N}\right)=\xi\left(y^{\prime},-y_{N}\right)$ for $y_{N}<0$. Assuming that $\Phi(z)=\Phi(-z)$, we may set $\hat{\Psi}_{1}(z)=\int_{-\infty}^{z_{1}} \Psi_{1}\left(s, z_{2}, z_{3}\right) \mathrm{d} s$, $\hat{\Psi}_{2}(z)=\int_{-\infty}^{z_{2}} \Psi_{2}\left(z_{1}, s, z_{3}\right) \mathrm{d} s$. Then $\hat{\Psi}_{1}, \hat{\Psi}_{2}$ have compact support and we may integrate by parts in (3.5.9) to obtain

$$
\begin{aligned}
\frac{\partial u^{\sigma}}{\partial \sigma}(x)= & -\sum_{i=1}^{2} \sigma^{-|\mathbf{b}|-1+2 b_{i}} \int_{\mathbb{R}^{3}} \hat{\Psi}_{i}\left(\frac{x-y}{\sigma^{\mathbf{b}}}\right) \frac{\partial^{2} u(y)}{\partial y_{i}^{2}} \mathrm{~d} y \\
& -\sigma^{-|\mathbf{b}|-1+b_{3}} \int_{\mathbb{R}^{3}} \Psi_{3}\left(\frac{x-y}{\sigma^{\mathbf{b}}}\right) \frac{\partial u(y)}{\partial y_{3}} \mathrm{~d} y .
\end{aligned}
$$


Integrals of the form $\int_{\mathbb{R}^{3}} \Psi_{*}\left(\frac{x-y}{\sigma^{\mathbf{b}}}\right) u_{*}(y) \mathrm{d} y$ with $u_{*} \in L^{2}\left(\mathbb{R}^{3}\right)$ can be estimated in $L^{4}\left(\mathbb{R}^{3}\right)$ using the Young inequality for convolutions as

$$
\left|\int_{\mathbb{R}^{3}} \Psi_{*}\left(\frac{\cdot-y}{\sigma^{\mathbf{b}}}\right) u_{*}(y) \mathrm{d} y\right|_{4} \leq \sigma^{(3 / 4)|\mathbf{b}|}\left|\Psi_{*}\right|_{4 / 3}\left|u_{*}\right|_{2} .
$$

Hence, by virtue of the choice of $\mathbf{b}$, we have

$$
\left|\frac{\partial u^{\sigma}}{\partial \sigma}\right|_{4} \leq C \sigma^{-1 / 2}\left(\left|\frac{\partial^{2} u}{\partial y_{1}^{2}}\right|_{2}+\left|\frac{\partial^{2} u}{\partial y_{2}^{2}}\right|_{2}+\left|\frac{\partial u}{\partial y_{3}}\right|_{2}\right), \quad\left|u^{\sigma}\right|_{4} \leq C \sigma^{-1 / 2}|u|_{2}
$$

and (4.37) follows from the inequality

$$
|u|_{4} \leq\left|u^{\sigma}\right|_{4}+\left|\int_{0}^{\sigma} \frac{\partial u^{\sigma^{\prime}}}{\partial \sigma^{\prime}} \mathrm{d} \sigma^{\prime}\right|_{4}
$$

\section{Proof of Theorems 3.4.1 and 3.4.2.}

Now, we can conclude the proof of the regularity of the solution $\theta$, proceeding first with the proof of Theorem 3.4.1.

We now define in particular the anisotropic spaces

$$
X^{p, q}=\left\{w \in L^{1}\left(\mathbb{R}_{+}^{N}\right): \int_{0}^{\infty}\left(\int_{\mathbb{R}^{N-1}}\left|w\left(y^{\prime}, y_{N}\right)\right|^{q} \mathrm{~d} y^{\prime}\right)^{p / q} \mathrm{~d} y_{N}<\infty\right\} .
$$

We can extend the functions defined on $\mathbb{R}_{+}^{N}$ by symmetry to $\mathbb{R}^{N}$, and use Corollary 3.5.2 of the previous section to obtain the embedding

$$
Y:=\left\{w \in L^{1}\left(\mathbb{R}_{+}^{N}\right): \partial_{N} w \in X^{p_{0}, q_{0}}, \quad \nabla_{y^{\prime}} w \in X^{p_{1}, q_{1}}\right\}
$$

in the space $C^{\alpha}\left(\mathbb{R}_{+}^{N}\right) \cap L^{\infty}\left(\mathbb{R}_{+}^{N}\right)$ of bounded $\alpha$-Hölder continuous functions for some $\alpha>0$, provided

$$
\frac{p_{0}^{\prime}}{p_{1} q_{0}}+\frac{1}{q_{1}}<\frac{1}{N-1}
$$

where $p_{0}^{\prime}$ is the conjugate exponent to $p_{0}$. As a direct consequence, we have

Lemma 3.5.3. Under the conditions of Lemma 3.4.5, we have $\nabla v \in L^{2}\left(0, T ; L^{\infty}\left(\mathbb{R}_{+}^{N}\right)\right)$.

Proof. The functions $\partial_{\ell} v$ for $\ell=1, \ldots, N-1$ belong to $L^{2}\left(0, T ; W^{2,2}\left(\mathbb{R}_{+}^{N}\right)\right)$, which is embedded into $L^{2}\left(0, T ; L^{\infty}\left(\mathbb{R}_{+}^{N}\right)\right)$ by classical Sobolev embedding theorems, see [8]. For $w(y, t)=\partial_{N} v(y, t)$ and a. e. $t \in(0, T)$, we have

$$
\begin{aligned}
& \left|\partial_{\ell} w(t)\right|_{X^{6,6}}=\left|\partial_{\ell} w(t)\right|_{6} \leq C\left(\left|\partial_{\ell} w(t)\right|_{2}+\left|\nabla \partial_{\ell} w(t)\right|_{2}\right) \quad \text { for } \quad \ell=1, \ldots, N-1, \\
& \left|\partial_{N} w(t)\right|_{X^{2, q}} \leq C\left(\left|\partial_{N} w(t)\right|_{2}+\left|\nabla_{y^{\prime}} \partial_{N} w(t)\right|_{2}\right)
\end{aligned}
$$

with a constant $C>0$ since for $N=2, W^{1,2}(\Omega) \subset L^{q}(\Omega)$, for every $q \geq 2$. Hence, (3.5.24) is fulfilled with $p_{0}=2, q_{0}=q, p_{1}=q_{1}=6$. Integrating over $t$ we conclude the proof of Lemma 3.5.3. 
This enables us to prove Theorems 3.4.1 and 3.4.2.

Proof of Theorems 3.4.1 and 3.4.2. We substitute in (3.4.1) new variables $y^{\prime}=x^{\prime}$, $y_{N}=x_{N}-g\left(x^{\prime}\right)$, and obtain for the new unknown function $\tilde{v}\left(y^{\prime}, y_{N}\right)=v\left(y^{\prime}, y_{N}+g\left(y^{\prime}\right)\right)$ the equation

$$
\int_{\mathbb{R}_{+}^{N}}\left(\tilde{v}_{t} \varphi+(\tilde{A} \nabla \tilde{v}+\tilde{B}) \cdot \nabla \varphi-\tilde{f} \varphi\right) \mathrm{d} y+\int_{\mathbb{R}^{N-1}} \tilde{h}\left(y^{\prime}, \tilde{v}\left(y^{\prime}, 0, t\right), v_{\Gamma}\left(y^{\prime}, t\right)\right) \varphi\left(y^{\prime}, 0\right) \mathrm{d} y^{\prime}=0
$$

for every $\varphi \in W^{1,2}\left(\mathbb{R}_{+}^{N}\right)$, where

$$
\begin{aligned}
\tilde{f}\left(y^{\prime}, y_{N}, t\right) & =f\left(y^{\prime}, y_{N}+g\left(y^{\prime}\right), t\right), \\
\tilde{v}_{\Gamma}\left(y^{\prime}, t\right) & =v_{\Gamma}\left(y^{\prime}, g\left(y^{\prime}\right), t\right), \\
\tilde{h}\left(y^{\prime}, v, v_{\Gamma}\right) & =h\left(y^{\prime}, g\left(y^{\prime}\right), v, v_{\Gamma}\right) \sqrt{1+\left|\nabla_{y^{\prime}} g\left(y^{\prime}\right)\right|^{2}}, \\
\tilde{A}\left(y^{\prime}, y_{N}\right) & =L^{T}\left(y^{\prime}\right) A\left(y^{\prime}, y_{N}+g\left(y^{\prime}\right)\right) L\left(y^{\prime}\right), \\
\tilde{B}\left(y^{\prime}, y_{N}, t\right) & =L^{T}\left(y^{\prime}\right) B\left(y^{\prime}, y_{N}+g\left(y^{\prime}\right), t\right),
\end{aligned}
$$

and where the matrix $L$ has the same form as in

$$
L=\left(\begin{array}{ccccc}
1 & 0 & \ldots & 0 & -\partial_{1} g \\
0 & 1 & \ldots & 0 & -\partial_{2} g \\
& & \ldots & & \\
0 & 0 & \ldots & 1 & -\partial_{N-1} g \\
0 & 0 & \ldots & 0 & 1
\end{array}\right)
$$

Theorem 3.4.1 now follows from Lemma 3.4.4. Theorem 3.4.2 is a consequence of Lemma 3.5.3.

We are now ready to prove Theorem 3.2.4.

Proof of Theorem 3.2.4. The nonlinear boundary condition is active only on the subsets $\Gamma_{j}$ of $\partial \Omega$ for $j=1, \ldots, n$.

We choose a covering of $\Omega,\left\{\Omega_{j}\right\}_{j=1}^{n}, \bar{\Omega} \subset \bigcup_{j=1}^{n} \Omega_{j}$ with the property that

$$
\Gamma_{j} \subset \Omega_{j}, \quad \Gamma_{i} \cap \bar{\Omega}_{j}=\emptyset \quad \text { for } i \neq j .
$$

We now find a smooth partition of unity $\left\{\lambda_{j}\right\}_{j=1}^{n}, \lambda_{j} \in C^{\infty}\left(\mathbb{R}^{N}\right)$ so that

$$
1=\sum_{j=1}^{n} \lambda_{j}(x) \quad \text { on } \bar{\Omega} \quad \text { such that } \operatorname{supp} \lambda_{j} \subset \bar{\Omega}_{j},
$$

(such a partition of unity exists, see for instance [8]). We set $v_{j}=\theta \lambda_{j}, f(x, t)=$ $r(\theta(x, t), c(x, t))$. After suitable deformations and rotations, we may assume that each set $\Omega_{j} \cap \bar{\Omega}$ can be extended to a domain $\tilde{\Omega}_{j}$ of the form (3.4.3), such that $\Gamma_{j} \subset \partial \tilde{\Omega}_{j}$. To derive the equation for $v_{j}$, we test the equation

$$
\int_{\Omega}\left(\theta_{t} \varphi+\nabla \theta \cdot \nabla \varphi-f(x, t) \varphi\right) \mathrm{d} x+\int_{\partial \Omega} h\left(x, \theta, \theta_{\Gamma}(x, t)\right) \varphi \mathrm{d} S=0
$$


by $\varphi=\lambda_{j} \tilde{\varphi}$, and obtain

$$
\int_{\tilde{\Omega}_{j}}\left(\left(v_{j}\right)_{t} \tilde{\varphi}+\nabla v_{j} \cdot \nabla \tilde{\varphi}+B_{j} \cdot \nabla \tilde{\varphi}-f_{j}(x, t) \tilde{\varphi}\right) \mathrm{d} x+\int_{\partial \tilde{\Omega}_{j}} h\left(x, v_{j}, v_{\Gamma j}(x, t)\right) \tilde{\varphi} \mathrm{d} S=0,
$$

with $B_{j}=-\theta \nabla \lambda_{j}, f_{j}=f \lambda_{j}-\nabla \theta \cdot \nabla \lambda_{j}, v_{\Gamma j}=\theta_{\Gamma} \lambda_{j}$. Here we have used the fact that $\lambda_{j}=1$ on $\Gamma_{j}$, and that $h$ is linear on $\partial \tilde{\Omega}_{j} \backslash \Gamma_{j}$.

The assumptions of Theorem 3.4.1 are satisfied; hence, each $v_{j}$ has the regularity $\left(v_{j}\right)_{t} \in L^{2}\left(\Omega_{j} \times(0, T)\right), v_{j} \in L^{2}\left(0, T ; W^{2,2}\left(\Omega_{j}\right)\right)$. From the formula

$$
\theta=\sum_{j=1}^{n} v_{j}
$$

it follows that $\theta_{t} \in L^{2}(\Omega \times(0, T)), \theta \in L^{2}\left(0, T ; W^{2,2}(\Omega)\right)$. Consequently, we may use Theorem 3.4.2 because we have the regularity of the coefficients needed from the assumptions and obtain $\nabla v_{j} \in L^{2}\left(0, T ; L^{\infty}\left(\Omega_{j}\right)\right)$ for each $j$. Hence $\nabla \theta \in L^{2}\left(0, T ; L^{\infty}(\Omega)\right)$, which is what we wanted to prove.

\subsection{Proof of continuous data dependence}

Let the hypotheses of Theorem 3.2.5 hold. In terms of $\left(\theta_{i}, u_{i}\right)$, equations (3.2.1)-(3.2.2) have the form, for a.e. $t \in(0, T)$ :

$$
\begin{aligned}
\int_{\Omega}\left(\left(\theta_{i}\right)_{t} \varphi+\nabla \theta_{i} \cdot \nabla \varphi-R\left(\theta_{i}, u_{i}\right) \varphi\right) \mathrm{d} x+\int_{\partial \Omega} h\left(x, \theta_{i}, \theta_{\Gamma i}(x, t)\right) \varphi \mathrm{d} S & =0 \\
\int_{\Omega}\left(G\left(\theta_{i}, u_{i}\right)_{t} \psi+\nabla u_{i} \cdot \nabla \psi-H\left(\theta_{i}, u_{i}\right) \nabla \theta_{i} \cdot \nabla \psi\right) \mathrm{d} x+\int_{\partial \Omega} b_{i}(x, t) \psi \mathrm{d} S & =0(
\end{aligned}
$$

for every test functions $\varphi, \psi \in V$, where $G, H$, and $R$ are defined by the identities

$$
F(\theta, G(\theta, u))=u, \quad H(\theta, u)=\frac{\partial F}{\partial \theta}(\theta, G(\theta, u)), \quad R(\theta, u)=r(\theta, G(\theta, u)) .
$$

Hypothesis 3.2.2 implies that $G, H, R$ are Lipschitz-continuous in both variables, $1 / d_{1} \leq$ $\partial_{u} G \leq 1 / d_{0}$.

Set

$$
U_{i}(x, t)=\int_{0}^{t} u_{i}(x, \tau) \mathrm{d} \tau, \quad u_{i}^{0}=F\left(\theta_{i}^{0}, c_{i}^{0}\right), \quad \bar{U}=U_{1}-U_{2} .
$$

We consider the difference of the equations (3.6.1) for $i=1$ and $i=2$, tested by $\varphi=\bar{\theta}$, integrate the difference of the equations (3.6.2) for $i=1$ and $i=2$ from 0 to $t$, and test by $\psi=\bar{U}_{t}$.

We denote by $C$ any constant independent of the solutions, and by $\varepsilon$ a small parameter, which will be suitably chosen. Since $\theta_{i}$ and $\theta_{\Gamma i}$ are uniformly bounded, we may assume that $h$ is Lipschitz-continuous in $\theta$ and $\theta_{\Gamma}$. 
Hence, using (3.4.8) for an appropriate $\varepsilon$, we obtain

$$
\begin{aligned}
\int_{\Omega}\left(\bar{\theta}_{t} \bar{\theta}+|\nabla \bar{\theta}|^{2}\right) \mathrm{d} x & \leq \int_{\Omega}\left(R\left(\theta_{1}, u_{1}\right)-R\left(\theta_{2}, u_{2}\right)\right) \bar{\theta} \mathrm{d} x+C \int_{\partial \Omega}\left(\left|\bar{\theta}_{\Gamma}\right|+|\bar{\theta}|\right) \bar{\theta} \mathrm{d} S \\
& \leq C \int_{\Omega}\left(|\bar{\theta}|+\left|\bar{U}_{t}\right|\right)|\bar{\theta}| \mathrm{d} x+C \int_{\partial \Omega}\left|\bar{\theta}_{\Gamma}\right|^{2} \mathrm{~d} S
\end{aligned}
$$

and

$$
\begin{aligned}
\int_{\Omega}\left(G\left(\theta_{1}, U_{1 t}\right)-G\left(\theta_{2}, U_{2 t}\right)\right) \bar{U}_{t}(x, t) \mathrm{d} x+\frac{1}{2} \frac{\mathrm{d}}{\mathrm{d} t} \int_{\Omega}|\nabla \bar{U}|^{2}(x, t) \mathrm{d} x \\
=\int_{\Omega}\left(\int_{0}^{t}\left(H\left(\theta_{1}, U_{1 t}\right) \nabla \theta_{1}-H\left(\theta_{2}, U_{2 t}\right) \nabla \theta_{2}\right)(x, \tau) \mathrm{d} \tau\right) \cdot \nabla \bar{U}_{t}(x, t) \mathrm{d} x \\
\quad-\int_{\partial \Omega}\left(\int_{0}^{t} \bar{b}(x, \tau) \mathrm{d} \tau\right) \bar{U}_{t}(x, t) \mathrm{d} S+\int_{\Omega}\left(G\left(\theta_{1}^{0}, u_{1}^{0}\right)-G\left(\theta_{2}^{0}, u_{2}^{0}\right)\right) \bar{U}_{t}(x, t) \mathrm{d} x \\
=\frac{\mathrm{d}}{\mathrm{d} t} \int_{\Omega}\left(\int_{0}^{t}\left(H\left(\theta_{1}, U_{1 t}\right) \nabla \theta_{1}-H\left(\theta_{2}, U_{2 t}\right) \nabla \theta_{2}\right)(x, \tau) \mathrm{d} \tau\right) \cdot \nabla \bar{U}(x, t) \mathrm{d} x \\
\quad-\int_{\Omega}\left(H\left(\theta_{1}, U_{1 t}\right) \nabla \theta_{1}-H\left(\theta_{2}, U_{2 t}\right) \nabla \theta_{2}\right) \cdot \nabla \bar{U}(x, t) \mathrm{d} x \\
\quad-\frac{\mathrm{d}}{\mathrm{d} t} \int_{\partial \Omega}\left(\int_{0}^{t} \bar{b}(x, \tau) \mathrm{d} \tau\right) \bar{U}(x, t) \mathrm{d} S+\int_{\partial \Omega} \bar{b}(x, t) \bar{U}(x, t) \mathrm{d} S \\
+\int_{\Omega}\left(c_{1}^{0}-c_{2}^{0}\right) \bar{U}_{t}(x, t) \mathrm{d} x .
\end{aligned}
$$

Integrating Eq. (3.6.5)-(3.6.4) with respect to $t$ and using the hypotheses on the data, we obtain

$$
\begin{aligned}
& \frac{1}{2} \int_{\Omega}|\bar{\theta}|^{2}(x, t) d x+\int_{0}^{t} \int_{\Omega}|\nabla \bar{\theta}(x, \tau)|^{2} \mathrm{~d} x \mathrm{~d} \tau \\
& \leq C \int_{0}^{t} \int_{\Omega}\left(|\bar{\theta}|+\left|\bar{U}_{t}\right|\right)|\bar{\theta}|(x, \tau) \mathrm{d} x \mathrm{~d} \tau+C \int_{0}^{t} \int_{\partial \Omega}\left|\bar{\theta}_{\Gamma}\right|^{2} \mathrm{~d} S \mathrm{~d} \tau+\frac{1}{2} \int_{\Omega}\left|\bar{\theta}^{0}\right|^{2}(x) d x \\
& \frac{1}{d_{1}} \int_{0}^{t} \int_{\Omega}\left|\bar{U}_{t}(x, \tau)\right|^{2} \mathrm{~d} x \mathrm{~d} \tau+\frac{1}{2} \int_{\Omega}|\nabla \bar{U}|^{2}(x, t) \mathrm{d} x \\
& \leq \quad C \int_{\Omega}\left(\int_{0}^{t}\left(\left(|\bar{\theta}|+\left|\bar{U}_{t}\right|\right)\left|\nabla \theta_{1}\right|+|\nabla \bar{\theta}|\right)(x, \tau) \mathrm{d} \tau\right)|\nabla \bar{U}(x, t)| \mathrm{d} x \\
& \quad+C \int_{0}^{t} \int_{\Omega}\left(\left(|\bar{\theta}|+\left|\bar{U}_{t}\right|\right)\left|\nabla \theta_{1}\right|+|\nabla \bar{\theta}|\right)(x, \tau)|\nabla \bar{U}(x, \tau)| \mathrm{d} x \mathrm{~d} \tau \\
& \quad+\int_{\partial \Omega}\left(\int_{0}^{t}|\bar{b}(x, \tau)| \mathrm{d} \tau\right)|\bar{U}(x, t)| \mathrm{d} S+\int_{0}^{t} \int_{\partial \Omega}|\bar{b}(x, \tau)||\bar{U}(x, \tau)| \mathrm{d} S \mathrm{~d} \tau \\
& \quad+C \int_{0}^{t} \int_{\Omega}|\bar{\theta}(x, \tau)|\left|\bar{U}_{t}(x, \tau)\right| \mathrm{d} x \mathrm{~d} \tau+C \int_{\Omega}\left|\bar{c}^{0}\right||\bar{U}(x, t)| \mathrm{d} x
\end{aligned}
$$


Using Hölder's and Young's inequalities, we may rewrite (3.6.6)-(3.6.7) as

$$
\begin{aligned}
&|\bar{\theta}(t)|_{2}^{2}+ \int_{0}^{t}|\nabla \bar{\theta}(\tau)|_{2}^{2} \mathrm{~d} \tau \\
& \leq C\left(\alpha(t)+\int_{0}^{t}|\bar{\theta}(\tau)|_{2}^{2} \mathrm{~d} \tau\right)+\varepsilon \int_{0}^{t}\left|\bar{U}_{t}(\tau)\right|_{2}^{2} \mathrm{~d} \tau \\
& \int_{0}^{t}\left|\bar{U}_{t}(\tau)\right|_{2}^{2} \mathrm{~d} \tau+|\nabla \bar{U}(t)|_{2}^{2} \\
& \leq C \int_{\Omega}\left(\int_{0}^{t}\left(\left(|\bar{\theta}|+\left|\bar{U}_{t}\right|\right)\left|\nabla \theta_{1}\right|+|\nabla \bar{\theta}|\right)(x, \tau) \mathrm{d} \tau\right)^{2} \mathrm{~d} x \\
&+C \int_{0}^{t}\left(\left(|\bar{\theta}|_{2}+\left|\bar{U}_{t}\right|_{2}\right)\left|\nabla \theta_{1}\right|_{\infty}+|\nabla \bar{\theta}|_{2}\right)(\tau)|\nabla \bar{U}(\tau)|_{2} \mathrm{~d} \tau \\
&+C\left(\alpha(t)+\int_{0}^{t}|\bar{\theta}(\tau)|_{2}^{2} \mathrm{~d} \tau\right) \\
&+\varepsilon\left(|\bar{U}(t)|_{2}^{2}+\int_{0}^{t} \int_{\partial \Omega}|\bar{U}(x, \tau)|^{2} \mathrm{~d} S \mathrm{~d} \tau+\int_{\partial \Omega}|\bar{U}(x, t)|^{2} \mathrm{~d} S\right)
\end{aligned}
$$

with $\alpha(t)$ defined by (3.2.6). The first two integrals on the right hand side of (3.6.9) will be estimated using Minkowski's inequality

$$
\begin{gathered}
\left(\int_{\Omega}\left(\int_{0}^{t}\left(\left(|\bar{\theta}|+\left|\bar{U}_{t}\right|\right)\left|\nabla \theta_{1}\right|+|\nabla \bar{\theta}|\right)(x, \tau) \mathrm{d} \tau\right)^{2} \mathrm{~d} x\right)^{1 / 2} \\
\leq \int_{0}^{t}\left(\int_{\Omega}\left(\left(|\bar{\theta}|+\left|\bar{U}_{t}\right|\right)\left|\nabla \theta_{1}\right|+|\nabla \bar{\theta}|\right)^{2}(x, \tau) \mathrm{d} x\right)^{1 / 2} \mathrm{~d} \tau \\
\leq \int_{0}^{t}\left(\left(|\bar{\theta}|_{2}+\left|\bar{U}_{t}\right|_{2}\right)\left|\nabla \theta_{1}\right|_{\infty}+|\nabla \bar{\theta}|_{2}\right)(\tau) \mathrm{d} \tau
\end{gathered}
$$

and Hölder's and Young's inequalities

$$
\begin{aligned}
& C \int_{0}^{t}\left(\left(|\bar{\theta}|_{2}+\left|\bar{U}_{t}\right|_{2}\right)\left|\nabla \theta_{1}\right|_{\infty}+|\nabla \bar{\theta}|_{2}\right)(\tau)|\nabla \bar{U}(\tau)|_{2} \mathrm{~d} \tau \\
& \quad \leq C \int_{0}^{t}\left(1+\left|\nabla \theta_{1}(\tau)\right|_{\infty}^{2}\right)|\nabla \bar{U}(\tau)|_{2}^{2} \mathrm{~d} \tau+\varepsilon \int_{0}^{t}\left(|\bar{\theta}|_{2}^{2}+\left|\bar{U}_{t}\right|_{2}^{2}+|\nabla \bar{\theta}|_{2}^{2}\right)(\tau) \mathrm{d} \tau
\end{aligned}
$$

respectively. Using the inequality $\frac{\mathrm{d}}{\mathrm{d} t}|\bar{U}(t)|_{2} \leq\left|\bar{U}_{t}(t)\right|_{2}$ a. e., we have in (3.6.9)

$$
|\bar{U}(t)|_{2}^{2} \leq\left(\int_{0}^{t}\left|\bar{U}_{t}(\tau)\right|_{2} \mathrm{~d} \tau\right)^{2}
$$

For the boundary terms in (3.6.9), we refer to the trace embedding (3.4.8). Using the inequality $\frac{\mathrm{d}}{\mathrm{d} t}|\bar{U}(t)|_{2} \leq\left|\bar{U}_{t}(t)\right|_{2}$ a. e., we have in (3.6.9)

$$
|\bar{U}(t)|_{2}^{2} \leq\left(\int_{0}^{t}\left|\bar{U}_{t}(\tau)\right|_{2} \mathrm{~d} \tau\right)^{2}, \quad \int_{0}^{t}|\bar{U}(\tau)|_{2}^{2} \mathrm{~d} \tau \leq \frac{1}{2} \int_{0}^{t}\left(t^{2}-\tau^{2}\right)\left|\bar{U}_{t}(\tau)\right|_{2}^{2} \mathrm{~d} \tau .
$$


Choosing $\varepsilon$ sufficiently small, we thus obtain from (3.6.8)-(3.6.10) the inequality

$$
\begin{aligned}
|\bar{\theta}(t)|_{2}^{2}+ & |\nabla \bar{U}(t)|_{2}^{2}+\int_{0}^{t}\left(|\nabla \bar{\theta}|_{2}^{2}+\left|\bar{U}_{t}\right|_{2}^{2}\right)(\tau) \mathrm{d} \tau \\
\leq & C\left(\alpha(t)+\int_{0}^{t}\left(1+\left|\nabla \theta_{1}\right|_{\infty}\right)^{2}\left(|\bar{\theta}|_{2}^{2}+|\nabla \bar{U}|_{2}^{2}\right)(\tau) \mathrm{d} \tau\right) \\
& +C\left(\int_{0}^{t}\left(1+\left|\nabla \theta_{1}\right|_{\infty}\right)\left(|\nabla \bar{\theta}|_{2}^{2}+\left|\bar{U}_{t}\right|_{2}^{2}\right)^{1 / 2}(\tau) \mathrm{d} \tau\right)^{2} .
\end{aligned}
$$

Inequality (3.6.12) is of the form

$$
v(t)+\int_{0}^{t} s^{2}(\tau) \mathrm{d} \tau \leq C\left(\alpha(t)+\int_{0}^{t} \beta^{2}(\tau) v(\tau) \mathrm{d} \tau+\left(\int_{0}^{t} \beta(\tau) s(\tau) \mathrm{d} \tau\right)^{2}\right)
$$

with

$$
\beta=1+\left|\nabla \theta_{1}\right|_{\infty} \in L^{2}(0, T), \quad v(t)=|\bar{\theta}(t)|_{2}^{2}+|\nabla \bar{U}(t)|_{2}^{2}, \quad s^{2}(t)=|\nabla \bar{\theta}(t)|_{2}^{2}+\left|\bar{U}_{t}(t)\right|_{2}^{2} .
$$

To estimate $v(t)$ and $s(t)$, we derive below in Lemma 3.6.2 a refined variant of the Gronwall lemma. Recall first the classical Gronwall estimate.

Lemma 3.6.1. Let $\alpha \in L^{\infty}(0, T)$ and $\gamma \in L^{1}(0, T)$ be given nonnegative functions, and let a nonnegative function $v \in L^{\infty}(0, T)$ satisfy for a. e. $t \in(0, T)$ the inequality

$$
v(t) \leq \alpha(t)+\int_{0}^{t} \gamma(\tau) v(\tau) \mathrm{d} \tau
$$

Then for a. e. $t \in(0, T)$ we have

$$
v(t) \leq \alpha(t)+\int_{0}^{t} \alpha(\tau) \gamma(\tau) \mathrm{e}^{\int_{\tau}^{t} \gamma(\sigma) \mathrm{d} \sigma} \mathrm{d} \tau \leq \sup _{0<\tau<t} \operatorname{ess} \alpha(\tau) \mathrm{e}^{\int_{0}^{t} \gamma(\sigma) \mathrm{d} \sigma} .
$$

Sketch of the proof. Multiplying both sides of (3.6.15) by $\mathrm{e}^{-\int_{0}^{t} \gamma(\sigma) d \sigma}$, we obtain the inequality

$$
\frac{\mathrm{d}}{\mathrm{d} t}\left(\mathrm{e}^{-\int_{0}^{t} \gamma(\sigma) \mathrm{d} \sigma} \int_{0}^{t} \gamma(\tau) v(\tau) \mathrm{d} \tau\right) \leq \mathrm{e}^{-\int_{0}^{t} \gamma(\sigma) \mathrm{d} \sigma} \alpha(t) \gamma(t)
$$

The assertion will follow integrating w.r.t. time the inequality above (for a proof see for instance [52]).

Lemma 3.6.1 can be viewed as a result of the fact that the $L^{\infty}$-norm of the function $v$ is bounded above by its weighted $L^{1}$-norm. We now show that an $L^{p}$-Gronwall estimate still holds if the $L^{\infty}$-norm on the left-hand side is replaced with an $L^{p}$-norm for $p>1$.

Lemma 3.6.2. Let $p>1$ and its conjugate exponent $p^{\prime}=p /(p-1)$ be fixed, and let $\alpha \in L^{\infty}(0, T), \gamma_{1} \in L^{1}(0, T)$, and $\gamma_{2} \in L^{p^{\prime}}(0, T)$ be given, $\gamma_{2}$ nonnegative. Let 
nonnegative functions $v \in L^{\infty}(0, T), s \in L^{p}(0, T)$ satisfy for a.e. $t \in(0, T)$ the inequality

$$
v(t)+\int_{0}^{t} s^{p}(\tau) \mathrm{d} \tau \leq \alpha(t)+\int_{0}^{t} \gamma_{1}(\tau) v(\tau) \mathrm{d} \tau+\left(\int_{0}^{t} \gamma_{2}(\tau) s(\tau) \mathrm{d} \tau\right)^{p}
$$

Then there exists a constant $M$ such that for a. e. $t \in(0, T)$ we have

$$
v(t)+\int_{0}^{t} s^{p}(\tau) \mathrm{d} \tau \leq M \sup _{0<\tau<t} \operatorname{ess} \alpha(\tau) .
$$

Proof. Set $G_{2}=\left(\int_{0}^{T} \gamma_{2}^{p^{\prime}}(\tau) \mathrm{d} \tau\right)^{1 / p^{\prime}}$. We fix $\delta$ such that for every $t \in[0, T]$ we have

$$
\left(\int_{(t-\delta)^{+}}^{t} \gamma_{2}^{p^{\prime}}(\tau) \mathrm{d} \tau\right)^{1 / p^{\prime}} \leq \frac{1}{2}
$$

and consider first $t \in[0, \delta]$. By Hölder's inequality we have

$$
\left(\int_{0}^{t} \gamma_{2}(\tau) s(\tau) \mathrm{d} \tau\right)^{p} \leq\left(\int_{0}^{t} \gamma_{2}^{p^{\prime}}(\tau) \mathrm{d} \tau\right)^{p-1} \int_{0}^{t} s^{p}(\tau) \mathrm{d} \tau \leq 2^{-p} \int_{0}^{t} s^{p}(\tau) \mathrm{d} \tau
$$

By substitution we obtain instead of (3.6.16), the following estimate:

$$
v(t)+\int_{0}^{t} s^{p}(\tau) \mathrm{d} \tau \leq \alpha(t)+\int_{0}^{t} \gamma_{1}(\tau) v(\tau) \mathrm{d} \tau+2^{-p} \int_{0}^{t} s^{p}(\tau) \mathrm{d} \tau
$$

and it is easy to see how to obtain (3.6.17) from Lemma 3.6.1.

We want to use an induction argument. To this aim, assume that inequality (3.6.17) is proved for $t \in[0, k \delta]$ with a constant $M=M_{k}$, and consider $t \in(k \delta,(k+1) \delta]$. We have

$$
\begin{aligned}
\left(\int_{0}^{t} \gamma_{2}(\tau) s(\tau) \mathrm{d} \tau\right)^{p} & =\left(\int_{0}^{t-\delta} \gamma_{2}(\tau) s(\tau) \mathrm{d} \tau+\int_{t-\delta}^{t} \gamma_{2}(\tau) s(\tau) \mathrm{d} \tau\right)^{p} \\
& \leq 2^{p-1}\left(\left(\int_{0}^{t-\delta} \gamma_{2}(\tau) s(\tau) \mathrm{d} \tau\right)^{p}+\left(\int_{t-\delta}^{t} \gamma_{2}(\tau) s(\tau) \mathrm{d} \tau\right)^{p}\right) \\
& \leq 2^{p-1} G_{2}^{p} \int_{0}^{t-\delta} s^{p}(\tau) \mathrm{d} \tau+\frac{1}{2} \int_{t-\delta}^{t} s^{p}(\tau) \mathrm{d} \tau \\
& \leq 2^{p-1} G_{2}^{p} M_{k} \sup _{0 \leq \tau \leq k \delta} \alpha(\tau)+\frac{1}{2} \int_{0}^{t} s^{p}(\tau) \mathrm{d} \tau
\end{aligned}
$$

The previous estimation yields:

$v(t)+\int_{0}^{t} s^{p}(\tau) \mathrm{d} \tau \leq \alpha(t)+\int_{0}^{t} \gamma_{1}(\tau) v(\tau) \mathrm{d} \tau+2^{p-1} G_{2}^{p} M_{k} \sup _{0 \leq \tau \leq k \delta} \alpha(\tau)+\frac{1}{2} \int_{0}^{t} s^{p}(\tau) \mathrm{d} \tau$

Again, we can see how this last estimate, by means of Lemma 3.6.1, yields (3.6.17), for $t \in(k \delta,(k+1) \delta]$. Having proved the assertion for the base case $k=1$ and the inductive step, we may assert the the proof by induction is concluded. 
We are able now to finish the proof of Theorem 3.2.5. Indeed, inequality (3.6.13) has the same form as in Lemma 3.6.2, with $p=2, \alpha$ replaced by $C \alpha, \gamma_{1}=C \beta^{2}$, and $\gamma_{2}=C^{1 / p} \beta$, with $v$ and $s$ given by (3.6.14). The assertion of Theorem 3.2.5 therefore follows from inequality (3.6.13) and Lemma 3.6.2. 
68CHAPTER 3. ANALYSIS OF A RELATED QUASILINEAR PARABOLIC SYSTEM 


\section{Chapter 4}

\section{Numerical results}

\subsection{Introduction}

In the present chapter we present some numerical simulations to show how the model developed and analysed in the previous chapters can be applied in concrete situations. In the last decade, the simulation of heat treatments has covered great importance in research as well as in industrial applications, being able to simulate more or less complex three-dimensional geometries. The practice of heat treatment simulations is used as a tool to deliver quantifying information about the characteristic properties of critical component regions regarding previous production steps and the initial state of the material.

Among the papers concerned with gas carburizing, we mention: [9], [24], [39], [44], [45], [46]. Often they are not concerned with the analysis of the underlying model, but they rather use the empirical trial and error method. Currently, there are several possibilities to perform gas carburizing under very different conditions, starting from the choice of the steel, the type of furnace and of gaseous atmosphere. For an overview of the very recent engineering literature dealing with gas carburizing and case hardening, we refer, for instance, to [38], [50] and references therein.

We investigate first the carburization stage and then we pass to consider the effect of an inhomogeneous carbon distribution in the workpiece on the kinetics of the phases under cooling, in comparison with the results obtained in presence of a homogenous carbon distribution.

We conclude bringing together these two main stages of the process, by showing some simulations performed for a two-dimensional configuration, a gear sector, where both carbon diffusion and quenching have been included. 
The simulations were performed on the basis of our model:

$$
\begin{aligned}
\rho \alpha \frac{\partial \theta}{\partial t}-\operatorname{div}(k \nabla \theta)=\rho L_{p} p_{t}+\rho L_{m} m_{t} & \text { in } \Omega \times(0, T) \\
\frac{\partial c}{\partial t}-\operatorname{div}((1-p-m) D(\theta, c) \nabla c)=0 & \text { in } \Omega \times(0, T) \\
p_{t}=(1-p-m) g_{1}(\theta, c) & \text { in } \Omega \times(0, T) \\
m_{t}=[\bar{m}(\theta, c)-m]_{+} g_{2}(\theta, c) & \text { in } \Omega \times(0, T) \\
-k \frac{\partial \theta}{\partial \nu}=h\left(\theta-\theta_{\Gamma}\right) & \text { on } \partial \Omega \times(0, T) \\
-(1-p-m) D(\theta, c) \frac{\partial c}{\partial \nu}=\beta\left(c-c_{p}\right) & \text { on } \partial \Omega \times(0, T) \\
\theta(x, 0)=\theta_{0} & \text { in } \Omega \\
c(x, 0)=c_{0} & \text { in } \Omega \\
p(0)=0 & \text { in } \Omega \\
m(0)=0 & \text { in } \Omega
\end{aligned}
$$

where

$$
\begin{aligned}
\bar{m}(\theta, c) & =\min \left\{m_{K M}(\theta, c), 1-p\right\} \\
m_{K M}(\theta, c) & =\left(1-e^{-c_{k m}(c)\left(M_{s}(c)-\theta\right)}\right) H\left(M_{s}(c)-\theta\right) .
\end{aligned}
$$

Denoting with $T$ the end time, we divide the interval $[0, T]$ as it is shown in Figure 4.1, as

$$
[0, T]=\left[t_{0}, t_{1}\right] \cup\left[t_{1}, t_{2}\right] \cup\left[t_{2}, T\right] .
$$

We do not take into account in this study the first time interval $\left[0, t_{0}\right]$, corresponding to the heating stage. In any case the heating phase presents no difficulty from the point of view of the mathematics and of the simulations.

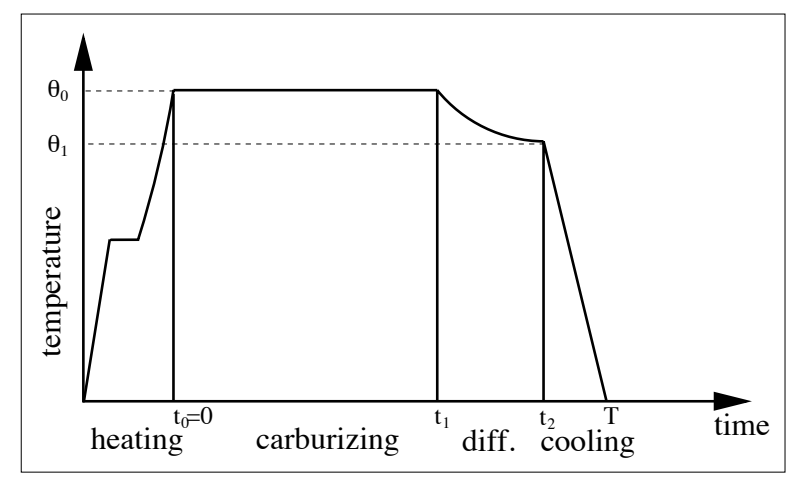

Figure 4.1: Schema of the gas carburizing cycle.

A general problem consists in determining the material parameters. As we do not have complete experimental data, we proceed as follows: we determine the parameters concerning the phase transitions from the TTT diagrams, for a certain steel, differing by carbon content. By interpolation, we obtain the necessary parameters as function 
of carbon content. We consider the low-alloyed steel 1320; its TTT diagrams for six different carbon contents (taken from [47], page 17) are shown in Figure 4.2.
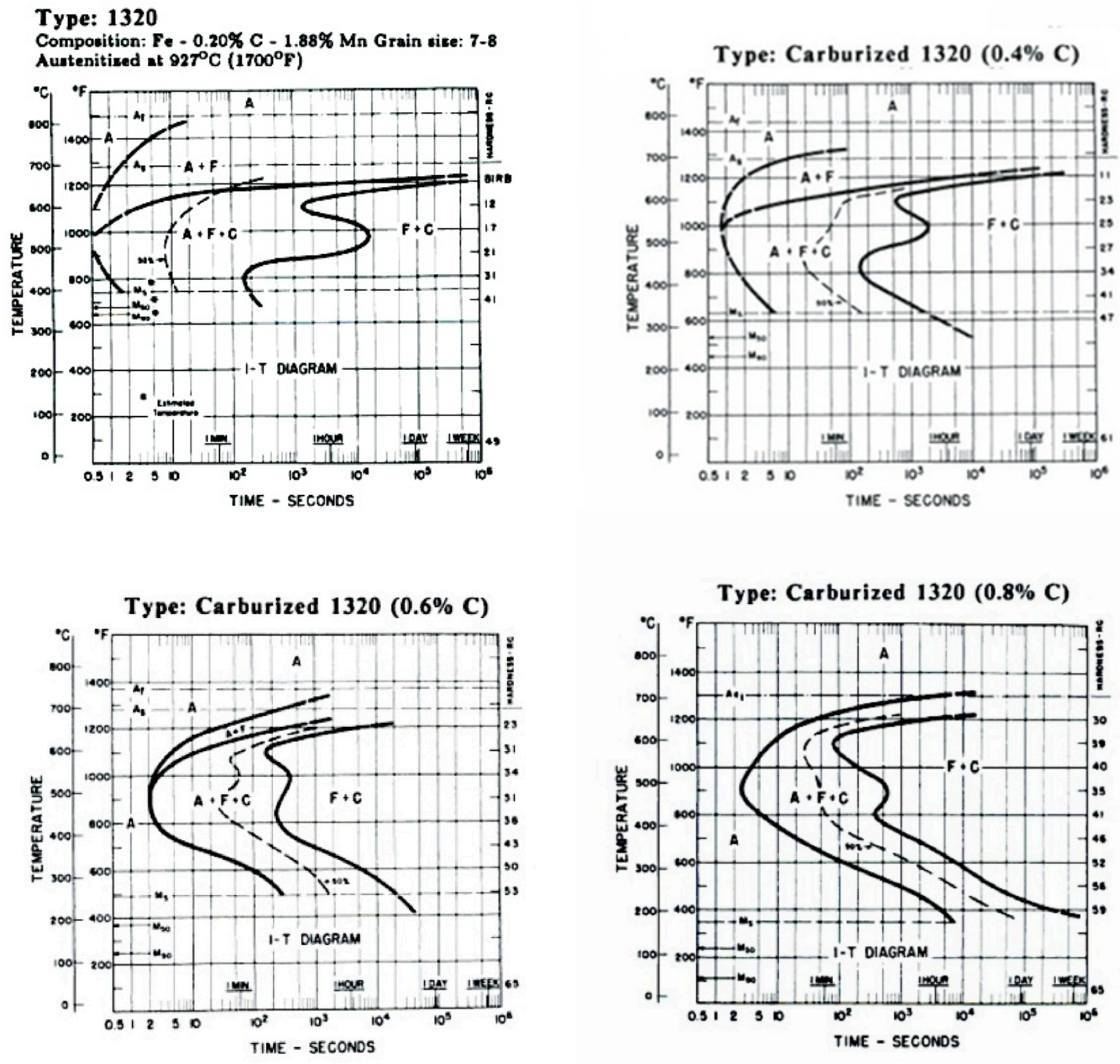

Type: Carburized $1320(1.0 \% \mathrm{C})$
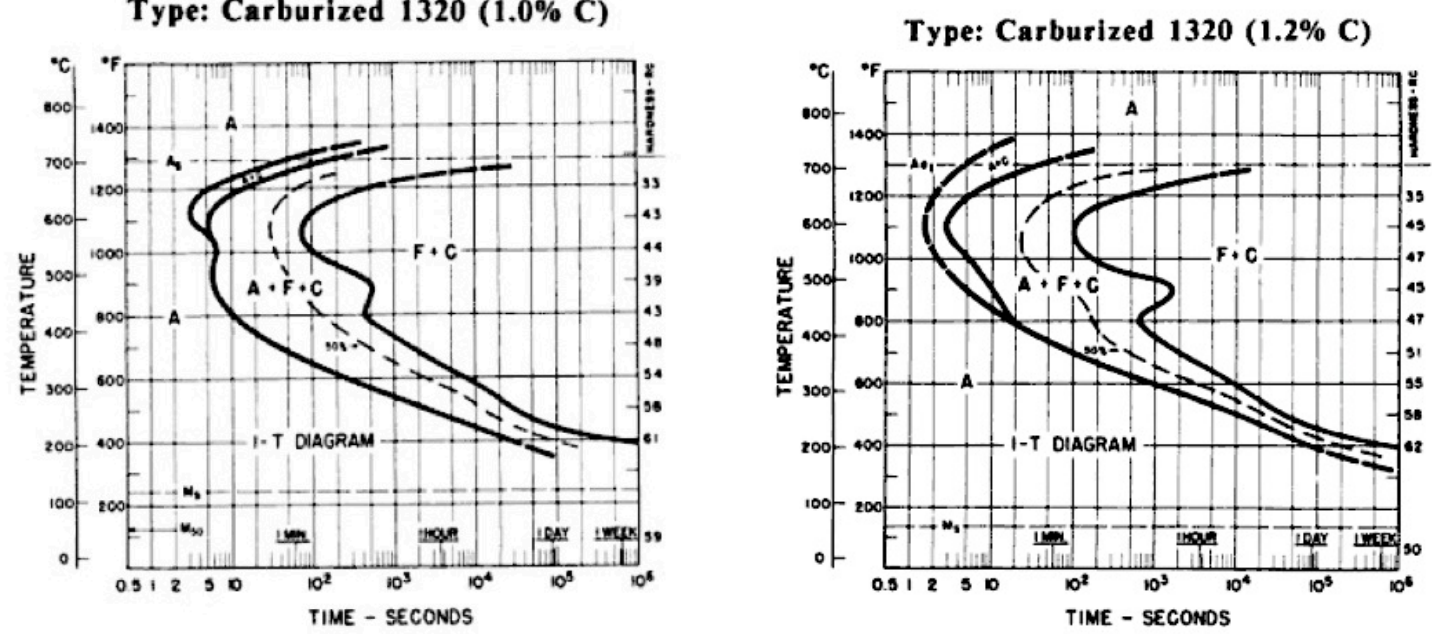

Figure 4.2: TTT diagrams for the steel 1320 for six different carbon contents (from [47]). 
From these diagrams we can directly read the values for $M_{s}$ and $M_{90}$ or $M_{50}$ depending on carbon content, from which we can extract the parameters $M_{s}(c)$ and $c_{k m}(c)$, entering in the formula (4.1.11). They are depicted in Figure 4.3.
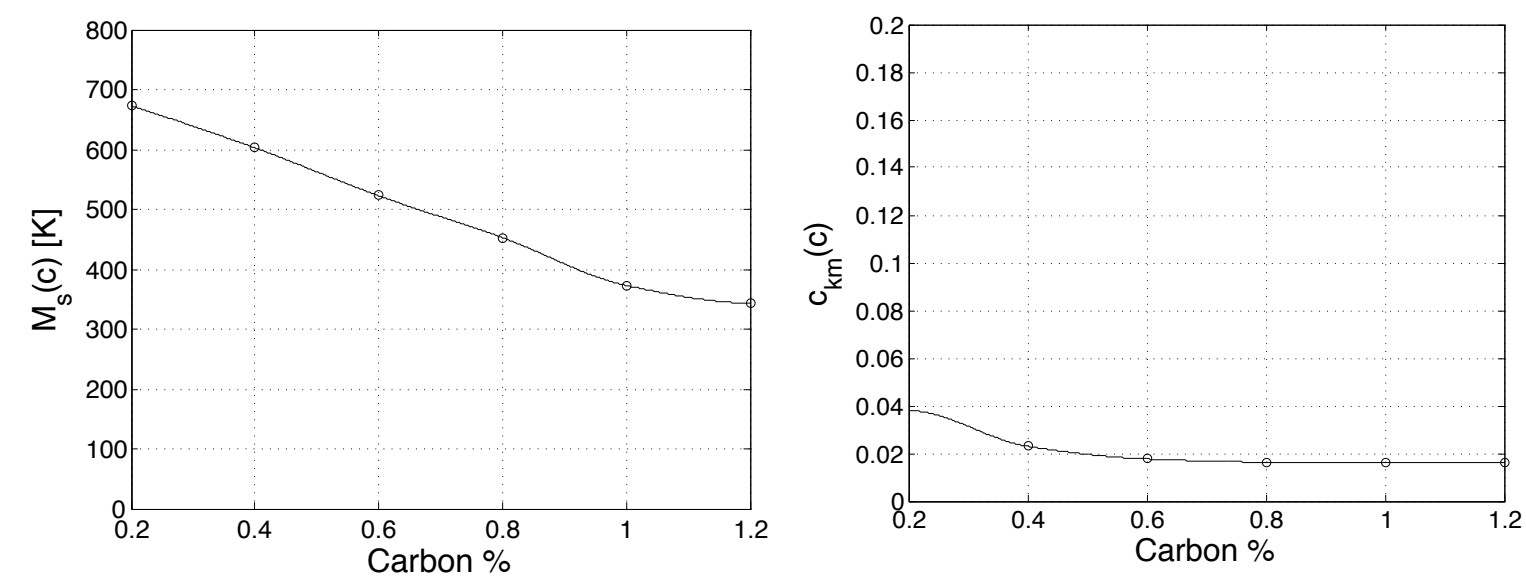

Figure 4.3: Plot of $M_{s}(c)$ and $c_{k m 3}(c)$, extracted from the TTT diagrams of Figure 4.2.

We point out that $\mathrm{F}+\mathrm{C}$ in Figure 4.2 corresponds, in our model, to what we denoted by 'pearlite' (a lamellar structure of ferrite and cementite). A close investigation of the diagrams shows that a small amount of ferrite is formed for carbon content smaller than $0.6 \%$. For carbon content larger that $0.8 \%$ there is a prior precipitation of carbides. These two effects are neglected in the following.

The function $g_{1}(\theta, c)$ in the equation (4.1.3) can be determined with the help of the TTT diagrams of Figure 4.2 arguing as follows. Since no martensite transformation happens above the temperature $M_{s}$ and no pearlite growth takes place below $M_{s}$, in the area $\theta>M_{s}$, we have $m=0$.

We take as limits of the integration interval $t_{s}$, where pearlite is $1 \%$ and $t_{f}$, where pearlite is $99 \%$. These two values can be derived from the TTT diagram, for a fixed temperature and for a fixed carbon content. Therefore, for fixed $c$, it holds:

$$
\begin{gathered}
\left(t_{f}-t_{s}\right) g_{1}(\theta, c)=\int_{t_{s}}^{t_{f}} g_{1}(\theta, c) d t=\int_{0.01}^{0.99} \frac{d p}{1-p}=[-\log (1-p)]_{0.01}^{0.99}=-\log \left(\frac{0.01}{0.99}\right) \\
\Longrightarrow g_{1}(\theta, c)=-\frac{1}{t_{f}-t_{s}} \log \left(\frac{0.01}{0.99}\right)
\end{gathered}
$$

The functions so obtained by interpolation between some discrete temperature values, for six different values of $c$ (those occurring in the TTT diagrams), are plotted in Figure 4.4 on the left. On the right can be seen a three dimensional plot of the function $g_{1}(\theta, c)$ by interpolation also in $c$.

The value for $g_{2}$ has been taken after the suggestion of [50] as $1 / 50 \mathrm{~s}^{-1}$. A constant value has been found to be sufficient to describe the kinetics of the phase transformations, since temperature and carbon effects are already incorporated in $\bar{m}$. 

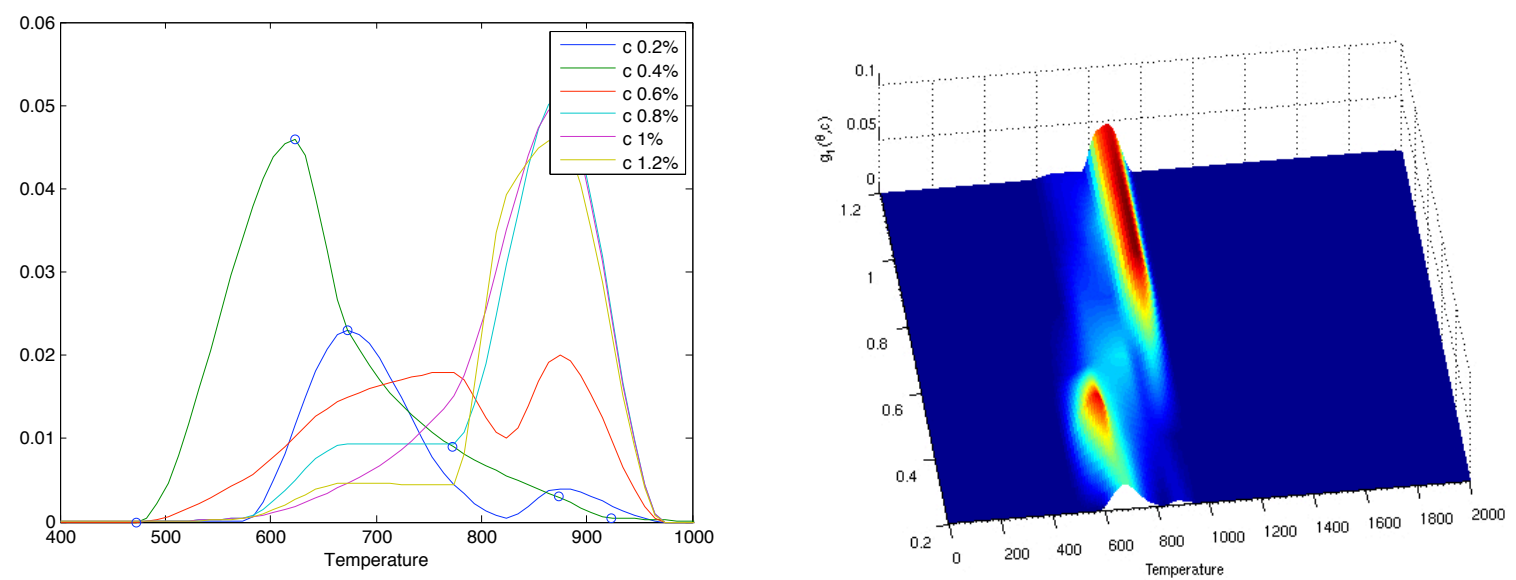

Figure 4.4: $g_{1}$ as a function of temperature, for different carbon contents (left). $g_{1}(\theta, c)$ after interpolation in $\theta$ and $c$.

Further necessary material parameters are listed in Table 4.1.

\begin{tabular}{|c|c|c|c|c|}
\hline$L_{p}$ & $L_{m}$ & $\rho$ & $\alpha$ & $k$ \\
\hline \hline $77000 \mathrm{~J} / \mathrm{Kg}$ & $82000 \mathrm{~J} / \mathrm{Kg}$ & $7800 \mathrm{~kg} / \mathrm{m}^{3}$ & $600 \mathrm{~J} / \mathrm{Kg} \mathrm{K}$ & $35 \mathrm{~W} / \mathrm{mK}$ \\
\hline
\end{tabular}

Table 4.1: Metallurgical parameters for the steel 1320.

The simulations were performed with Comsol Multiphysics, a software oriented to the solution of PDEs based on the finite element method. The unit system SI is adopted.

\subsection{Carburization}

The desired carbon profile is achieved through the carburization and diffusion stages. The two main factors influencing the final case depth of carbon are the temperature and the duration of carburization. Also significant for the final result is the presence of a diffusion period before quenching. Therefore we focused the simulations on the effect of time and temperature of carburization and on the consecutive diffusion stage. We start considering as sample geometry a one-dimensional domain $\Omega=[0,0.05]$, assuming the point 0 to be the boundary and the other extreme of the interval the 'inner part' of the workpiece. The initial temperature of carburization, $\theta_{0}$, is chosen above the austenitization temperature, such that we may assume the workpiece to be homogeneously austenitic, at the beginning.

The values $c_{0}$, the initial carbon concentration and $c_{p}$, the carbon potential, are both given in weight \%. The expression for the diffusion coefficient of carbon in austenite is

$$
D(\theta, c)=0.000047 \exp (-1.6 c-(37000-6600 c) /(1.987 \theta)) \mathrm{m}^{2} / \mathrm{s} .
$$


This formula is taken from Ref. [44] and the value for $\beta=6 \cdot 10^{-7}$ as well. A plot of $D(\theta, c)$ is depicted in Figure 4.5 (a comparison of different formulas for this coefficient can be found in [41]).
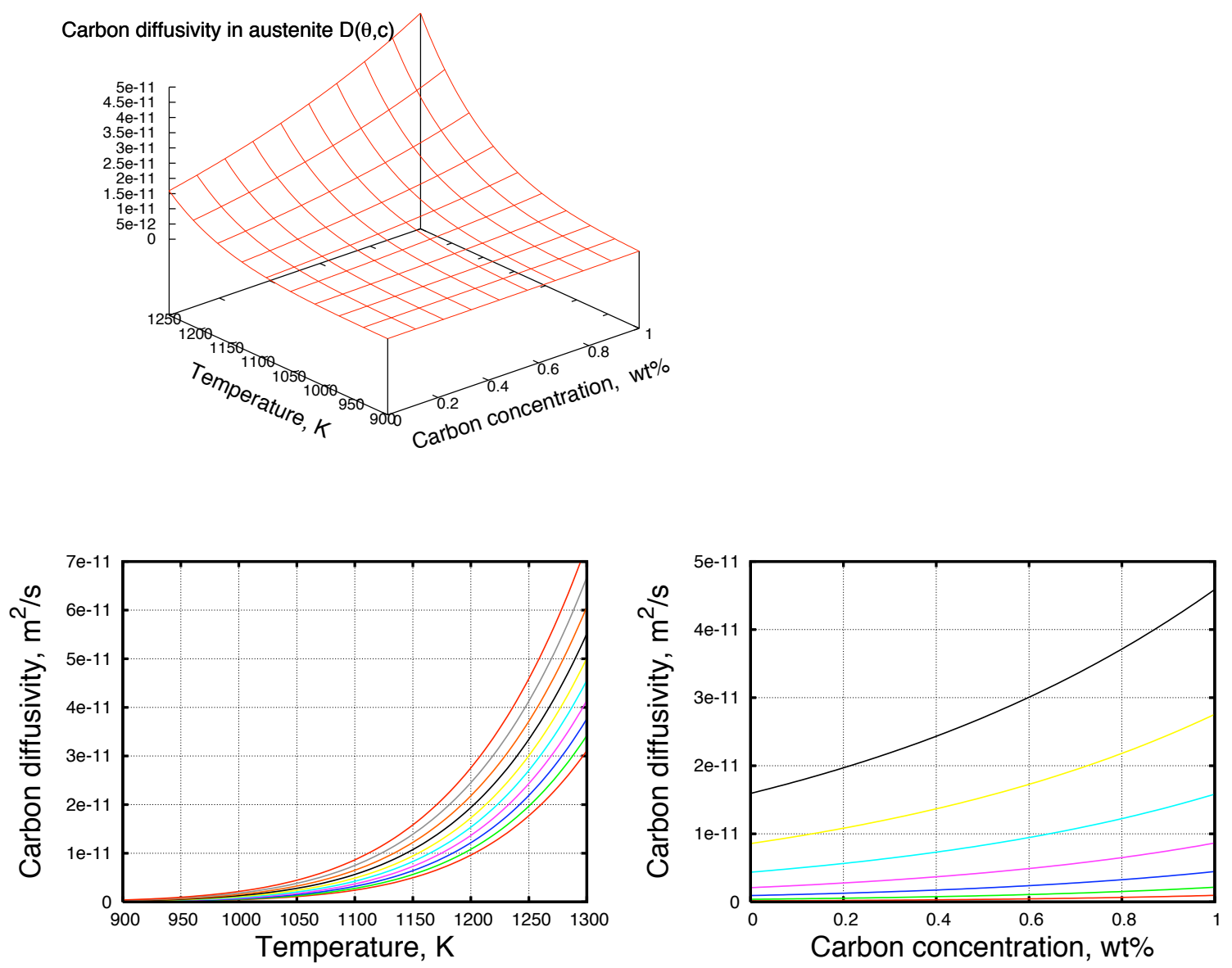

Figure 4.5: Coefficient of carbon diffusion in austenite (upper figure); surface representation as a function of temperature (below on the left), as a function of carbon content (below on the right). According to [44].

\section{Effect of temperature}

Regarding the carburized case, it can vary between a few tenths and several millimitres and must be matched to the function and the size of the component. Its thickness depends strongly on duration and temperature of carburization.

The results, plotted in Figure 4.6 show a good agreement with the ones contained in [44] by C.A. Stickels, which are based on the experiments conducted in [17]. The results obtained in [44] are plotted in Figure 4.7.

The maximum rate at which carbon can be added to steel is limited by the rate of diffusion of carbon in austenite. This diffusion rate increases greatly with increasing temperature; the rate of carbon addition at $1228 \mathrm{~K}$ is about $40 \%$ greater than at 1144 
$\mathrm{K}$. It is mainly for this reason that the most common temperature for gas carburizing is 1200 to $1250 \mathrm{~K}$. For shallow-case carburizing in which the case depth must be kept within a specified narrow range, lower temperature are frequently used, because case depth can be more accurately controlled with the slower carburizing rates obtained with low temperatures.

The indication 'effective case depth' specifies the depth at which the carbon content is $0.4 \%$. Generally, "case depth" is specified as the depth below the surface at which a defined value of some property occurs. A case depth to a hardness of $50 \mathrm{HRC}$ and a case depth to a carbon content of $0.4 \%$ are examples of a specifications for an effective case depth.

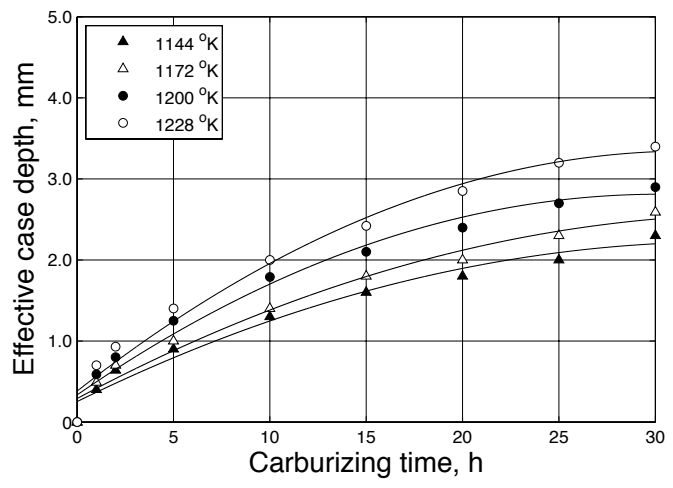

\begin{tabular}{|c|c|c|c|c|}
\hline $\begin{array}{c}\text { Time, } \\
\mathrm{h}\end{array}$ & $\begin{array}{c}1144^{\circ} \mathrm{K} \\
\mathrm{mm}\end{array}$ & $\begin{array}{c}1172^{\circ} \mathrm{K} \\
\mathrm{mm}\end{array}$ & $\begin{array}{c}1200^{\circ} \mathrm{K} \\
\mathrm{mm}\end{array}$ & $\begin{array}{c}1228^{\circ} \mathrm{K} \\
\mathrm{mm}\end{array}$ \\
\hline 1 & 0.41 & 0.49 & 0.59 & 0.70 \\
2 & 0.64 & 0.70 & 0.80 & 0.93 \\
5 & 0.90 & 1.00 & 1.25 & 1.40 \\
10 & 1.30 & 1.40 & 1.79 & 2.00 \\
15 & 1.60 & 1.80 & 2.10 & 2.42 \\
20 & 1.80 & 2.00 & 2.40 & 2.85 \\
25 & 2.00 & 2.30 & 2.70 & 3.20 \\
30 & 2.30 & 2.59 & 2.90 & 3.40 \\
\hline
\end{tabular}

Data derived from the simulations.

Figure 4.6: Plot of effective case depth versus carburizing time at four selected temperatures. Graph based on data in table.

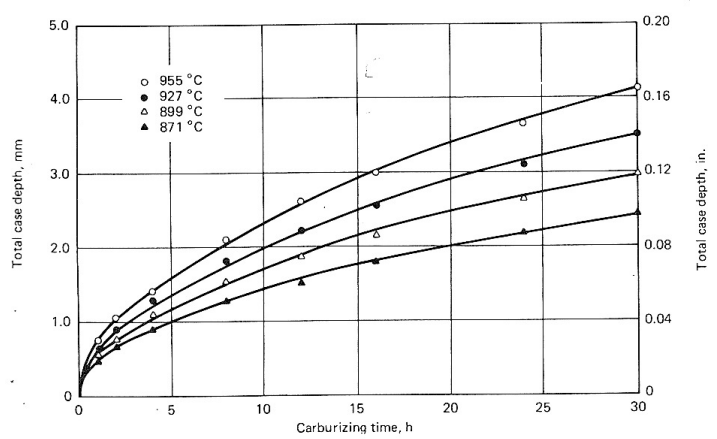

Figure 4.7: Picture taken from [44] depicting the total case depth versus carburizing time at four selected temperatures, based on the experimental data contained in [17].

\section{Effect of time}

The depth of case depth achieved versus time is not a linear relationship, but it is more like an inverse proportionality relationship to the fifth power. Referring again to the Table in Figure 4.6, it can be seen that the gain in case depth with increasing time for fixed carburizing temperature is a matter of rapidly diminishing returns. For example, at any of the four temperatures shown, to double the case depth obtained in 
1 hour, the time is approximately quintupled.

\section{Effect of the diffusion stage}

We examine here the effect of the further carbon diffusion inside the workpiece, during the diffusion period of carbon into the workpiece allowed sometimes before quenching (referring to Figure 4.1 , it is the interval $\left[t_{1}, t_{2}\right]$ ). The carbon profiles at time $t_{2}$ are depicted in Figure 4.8, depending on the length of the interval $\left[t_{1}, t_{2}\right]$, for different values of $t_{2}$.

We recall that the carbon potential assumed in the interval $\left[0, t_{1}\right]$ is $c_{p}=1.2$. The green line, which represents the profile of carbon along the interval at time $t_{1}$, is a monotonically decreasing function, starting approximately from the value 1.2 , as expected. Then this profile falls gradually as $t_{2}$ increases in presence of a lower carbon potential $c_{p}$ and of a lower external temperature $\theta_{\text {out }}$; the corresponding profiles after different end times $t_{2}$ are indicated with the blue, red and black lines. The rate of decline in this stage is the effect of the diffusion within the steel, the surface reaction rate and the rate at which the atmosphere composition changes. The conditions of the process, specified in Table 4.2, are chosen in compatibility to those used in [45]. Indeed, the results of our simulations have been compared with those contained in [45], which are shown in Figure 4.9. Here we see the computed carbon gradient in the workpiece at the end of the carburization stage (line 1) and at the end of a diffusion stage of $5000 \mathrm{~s}$. (line 2). The process parameters used by the author of [45], C. A. Stickels, are specified in the same figure. From the comparison between Figure 4.8 and 4.9 , we can conclude that the results agree qualitatively and quantitatively.

\begin{tabular}{|c|c|c|c|c||c|c||c|c|}
\hline Time & $t_{2}=t_{1}$ & $t_{2}^{\prime}$ & $t_{2}^{\prime \prime}$ & $t_{2}^{\prime \prime \prime}$ & $\theta_{0}$ & $\theta_{\Gamma}$ & $c_{p}$ & $\beta$ \\
\hline & $8 \mathrm{~h}$ & $24 \mathrm{~h}$ & $48 \mathrm{~h}$ & $72 \mathrm{~h}$ & $1158 \mathrm{~K}$ & $800 \mathrm{~K}$ & 0.8 & $2 \cdot 10^{-7}$ \\
\hline
\end{tabular}

Table 4.2: Specified carburizing conditions in the time interval $\left[t_{1}, t_{2}\right]$ for $t_{1}=8 \mathrm{~h}$.
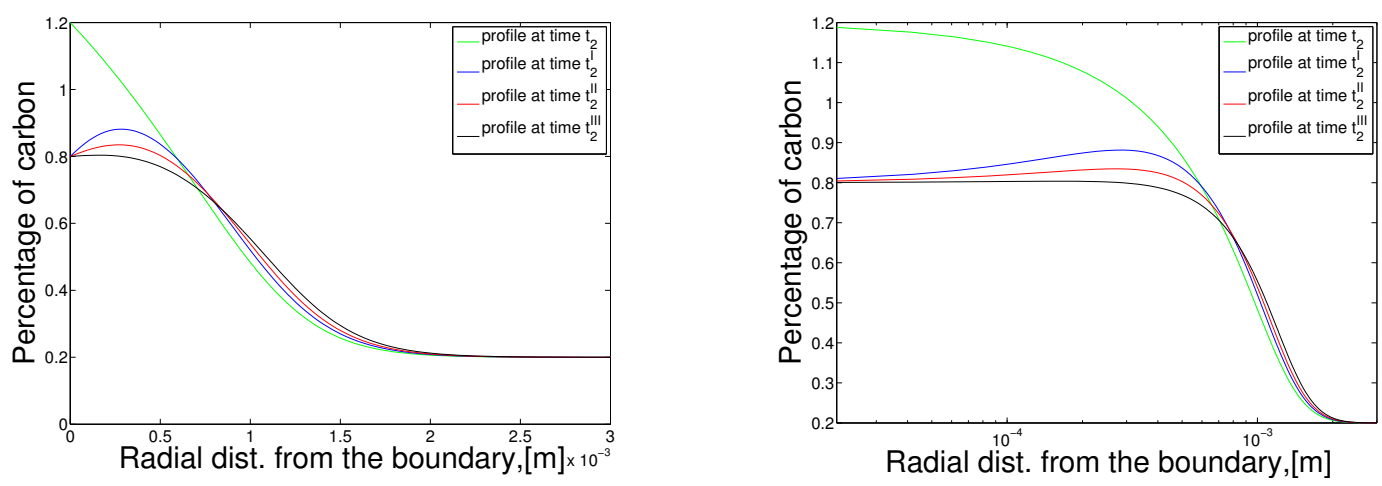

Figure 4.8: Computed carbon gradient at the end of different diffusion times on the lenght of the radius (left) and in an enlargement around the boundary (right). 


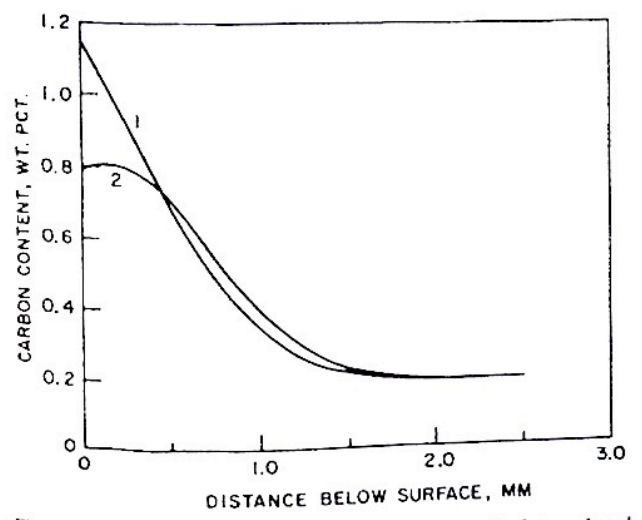

Fig. 8-Computed carton gradients in parts at the end of stage 1 and slage 2 for the second batch furmace example.
Specified carburizing conditions referred to the figure on the left:

\begin{tabular}{c|c|c} 
& Stage 1 & Stage 2 \\
\hline Temperature, K & 2113 & 1158 \\
\hline Carbon potential $c_{p}$ & 1.15 & 0.8 \\
\hline$\beta$ & $2 \cdot 10^{-7}$ & $2 \cdot 10^{-7}$ \\
\hline Time, h & 4 & 1.5
\end{tabular}

Figure 4.9: Picture taken from [45], showing the carbon gradient in a workpiece after carburization (line 1) and additional diffusion (line 2), with the corresponding process parameters in the table on the right.

\subsection{Quenching}

Now we consider separately the cooling stage of the process, which takes place at a different time scale than the diffusion stage. To this end, we need to specify further process parameters :

\begin{tabular}{|c|c|c|c|}
\hline$h$ & $\theta_{0}$ & $\theta_{\Gamma}$ & $T-t_{2}$ \\
\hline $20000 \mathrm{~W} / \mathrm{m}^{2} \mathrm{~K}$ & $1228 \mathrm{~K}$ & $300 \mathrm{~K}$ & $500 s$ \\
\hline
\end{tabular}

Table 4.3: Further necessary process parameters.

To understand the effect of an inhomogeneous carbon distribution in the workpiece, we first performed simulations for two homogeneous carbon contents, $0.5 \%$ and $0.2 \%$, with $\Omega=[0,0.05]$. The results are respectively plotted in Figure 4.10 and 4.11 . We assumed a heat flux active in $\mathrm{x}=0$ (boundary) while at the other extreme of the interval (inside the body), we assume thermal insulation. The first picture shows the profile of austenite, pearlite and martensite over the interval at the end of quenching; the deviation of the workpiece temperature from the external temperature ( $\mathrm{T} /$ Tout) is also depicted. The following five pictures represent the phases and temperature evolution in time at five selected points, $a, b, c, d, e$ at increasing distance from the boundary. 


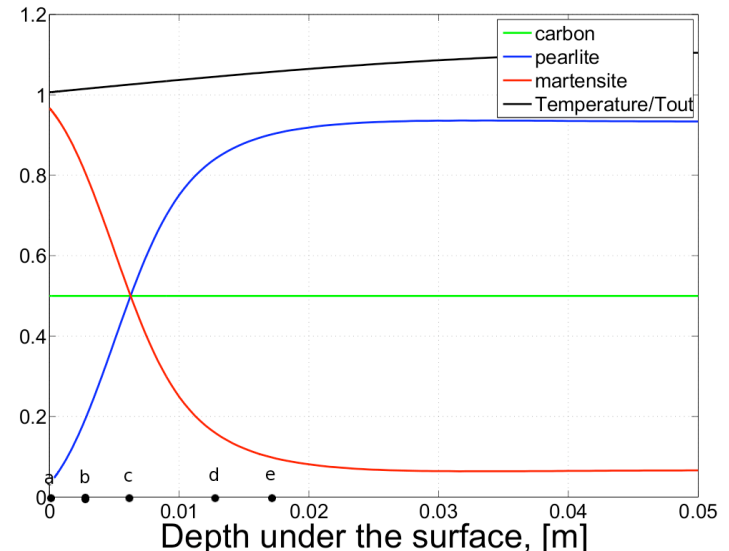

Final phase distributions.

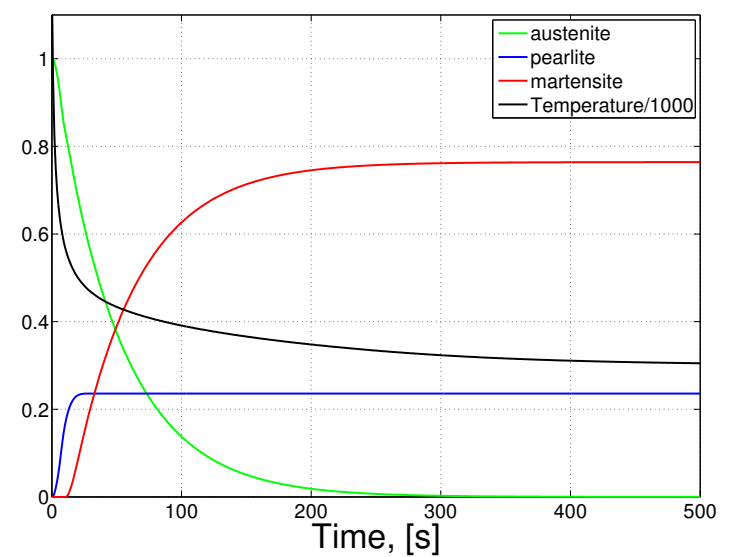

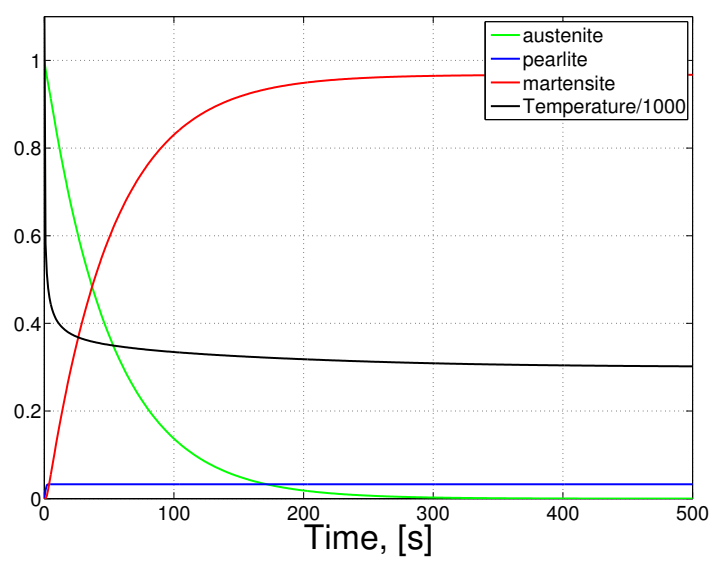

Phases and temperature evolution at point $a$.

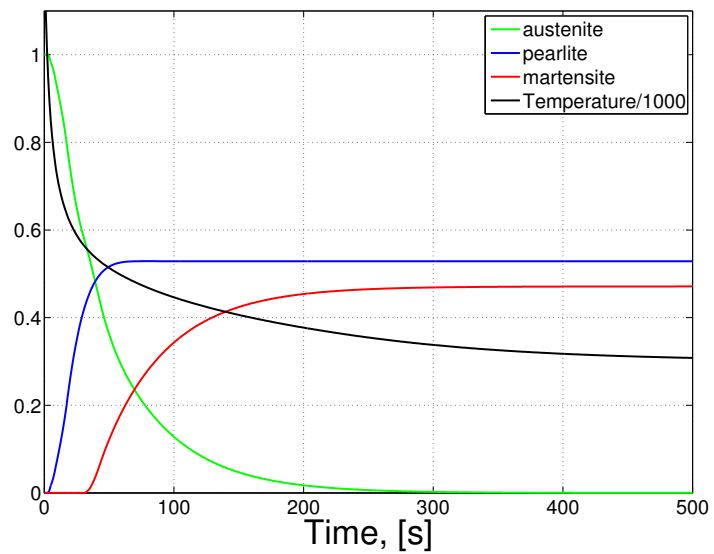

Phases and temperature evolution at point $b$. Phases and temperature evolution at point $c$.
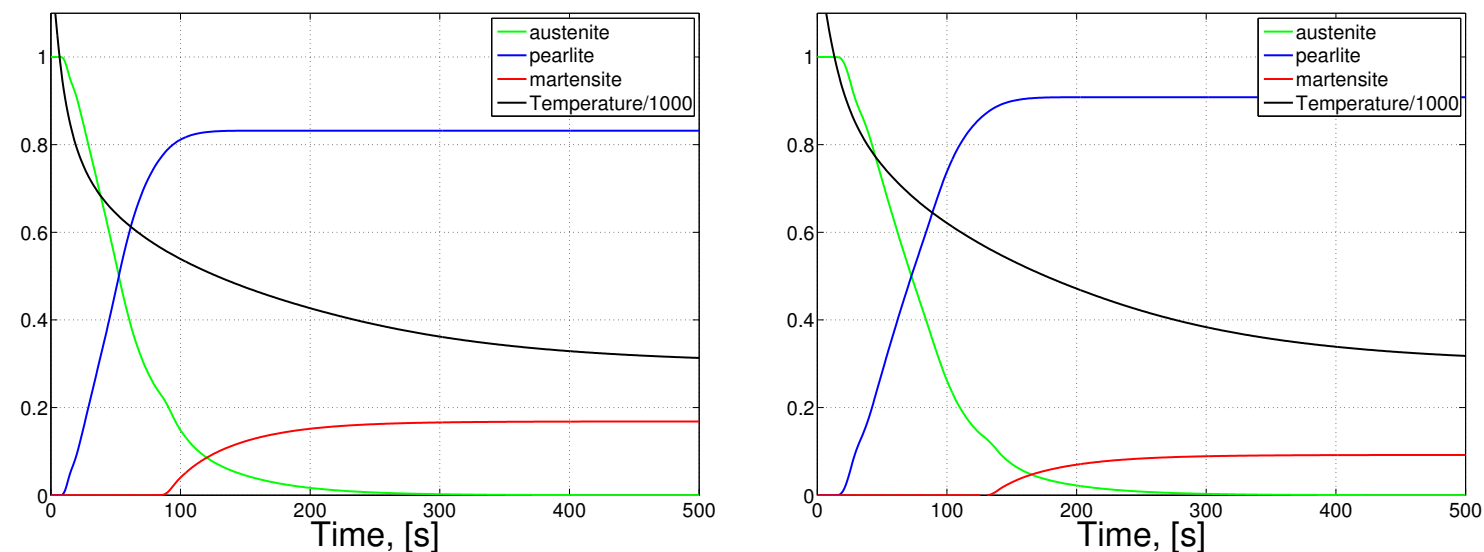

Phases and temperature evolution at point $d$. Phases and temperature evolution at point $e$.

Figure 4.10: Final phases distribution for a homogeneous carbon content $0.5 \%$ (upper left) and five pictures with the evolution of phases and temperature during quenching at five selected points. 


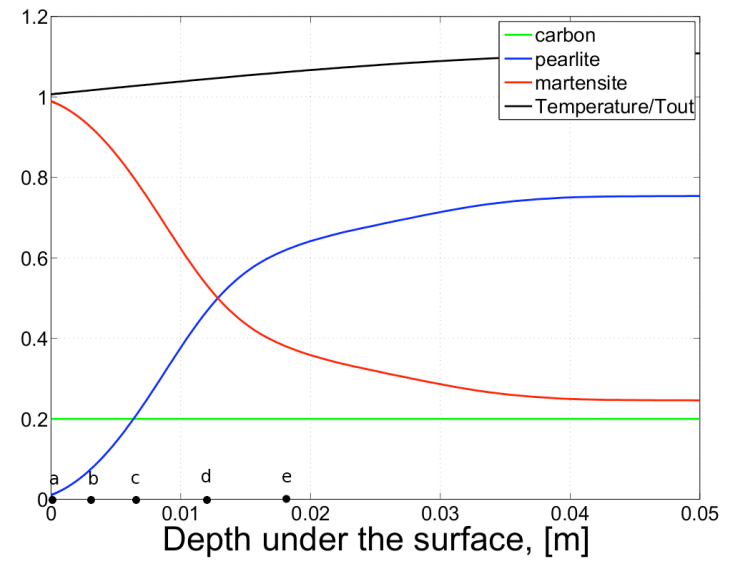

Final phase distributions.

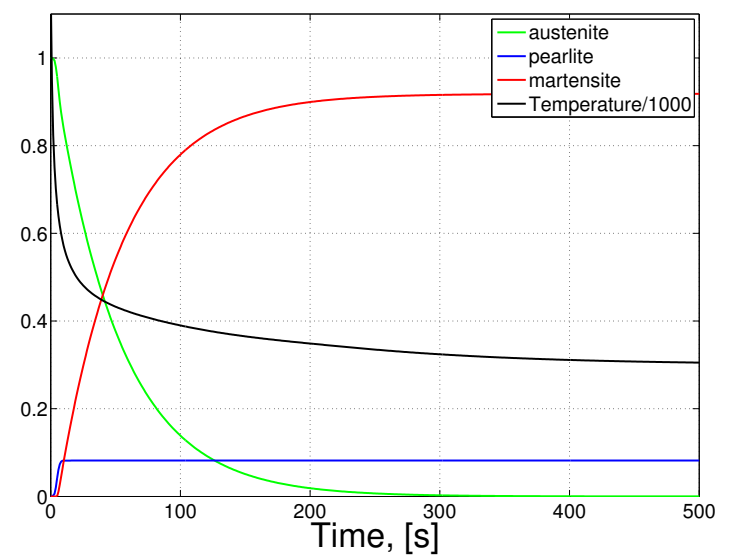

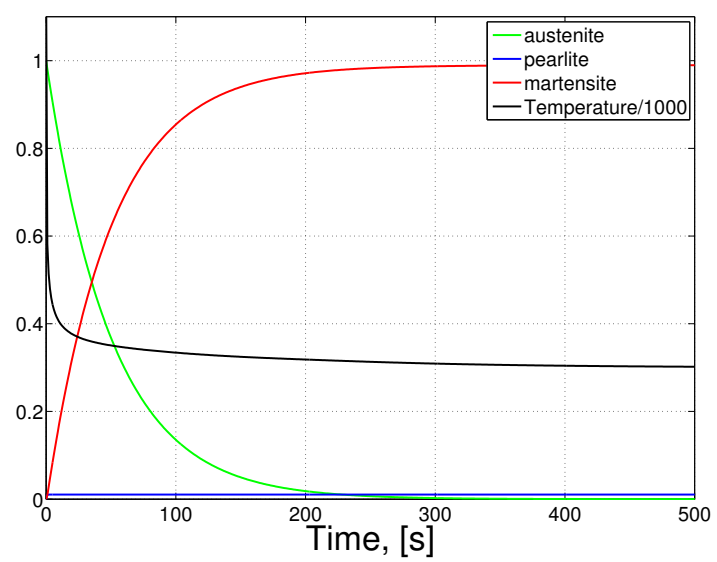

Phases and temperature evolution at point $a$.

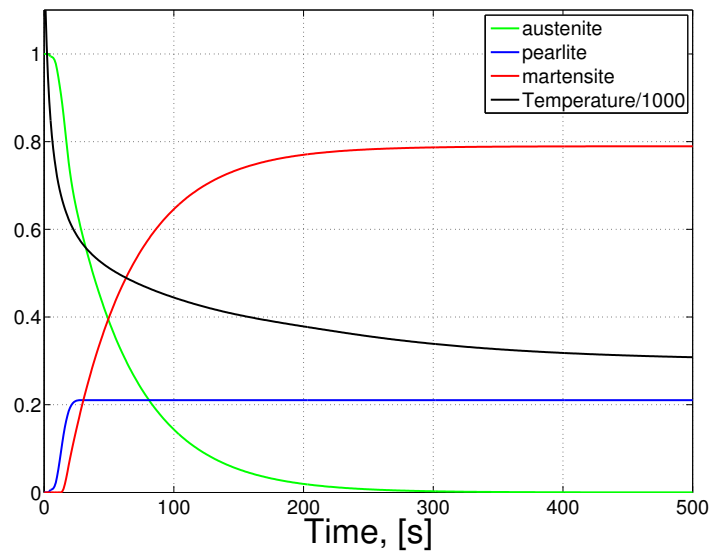

Phases and temperature evolution at point $b$. Phases and temperature evolution at point $c$.
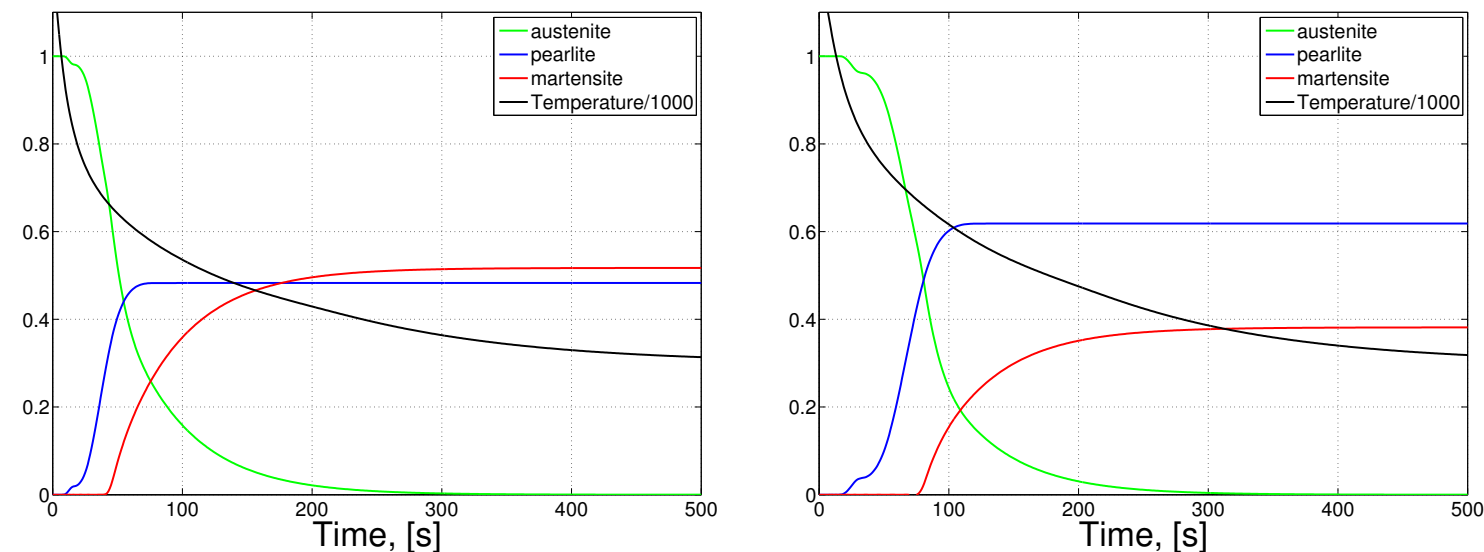

Phases and temperature evolution at point $d$. Phases and temperature evolution at point $e$.

Figure 4.11: Final phases distribution for a homogeneous carbon content $0.2 \%$ (upper left) and five pictures with the evolution of phases and temperature during quenching at five selected points.

Through the line depicting $\mathrm{T} /$ Tout, it is visible how the cooling rate decreases from the boundary to the interior of the workpiece. Correspondingly, in both cases, we 
note the usual diversification in the formation of pearlite and martensite: the faster the quenching is, the higher is the amount of marteniste and the lower the one of pearlite formed. We can also see how the phases are monotone function of the spacial coordinate. The kinetic behaviours of the phases in the case of $0.2 \%$ carbon and $0.5 \%$ carbon are similar, but there are differences in the quantity of phase produced.

Now we pass to the case of a workpiece with inhomogeneous carbon distribution: the carbon profile decreases from $0.5 \%$ near the boundary to $0.2 \%$ in the middle of the body, which corresponds to the green profile depicted in the first picture of Figure 4.12. As in the previous Figure, we show in Figure 4.12 the resulting final phase distributions over the depth and five plots for the five points selected to observe the time evolution.

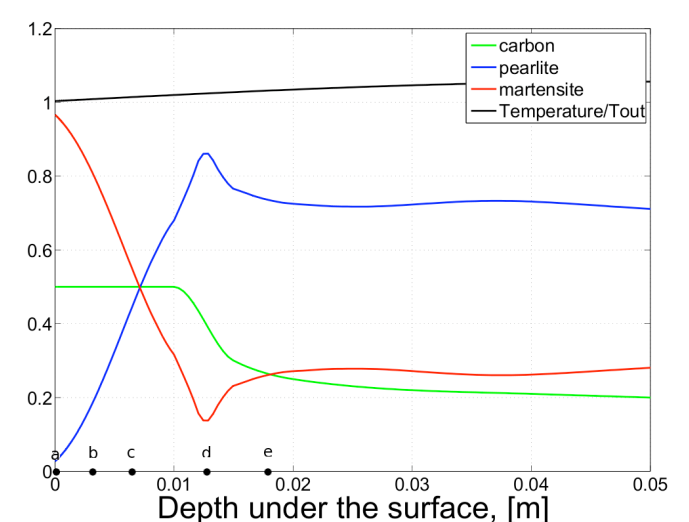

Final phase distributions.

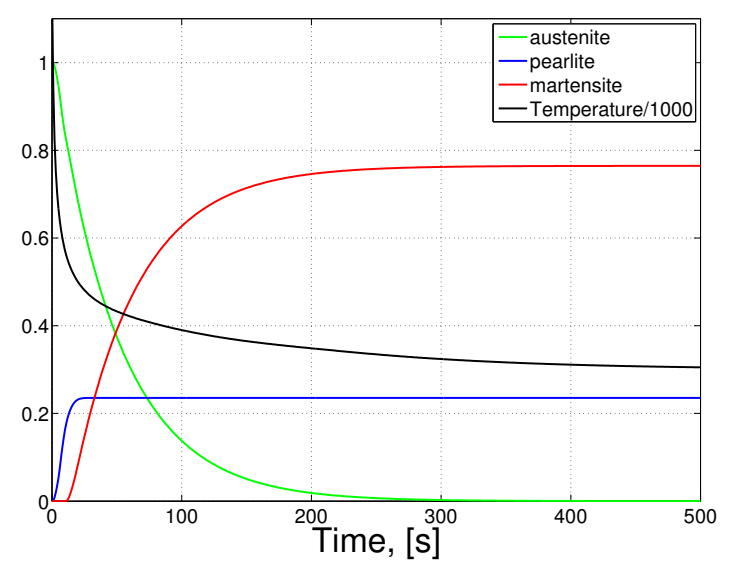

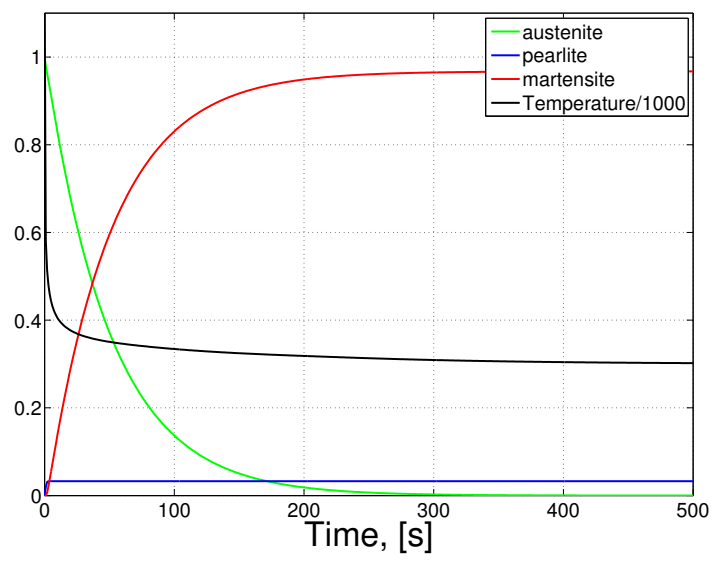

Phases and temperature evolution at point $a$.

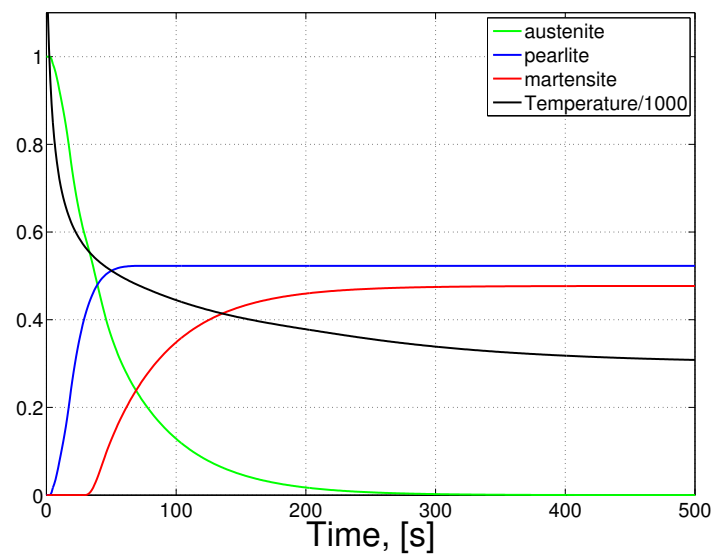

Phases and temperature evolution at point $b$. Phases and temperature evolution at point $c$. 

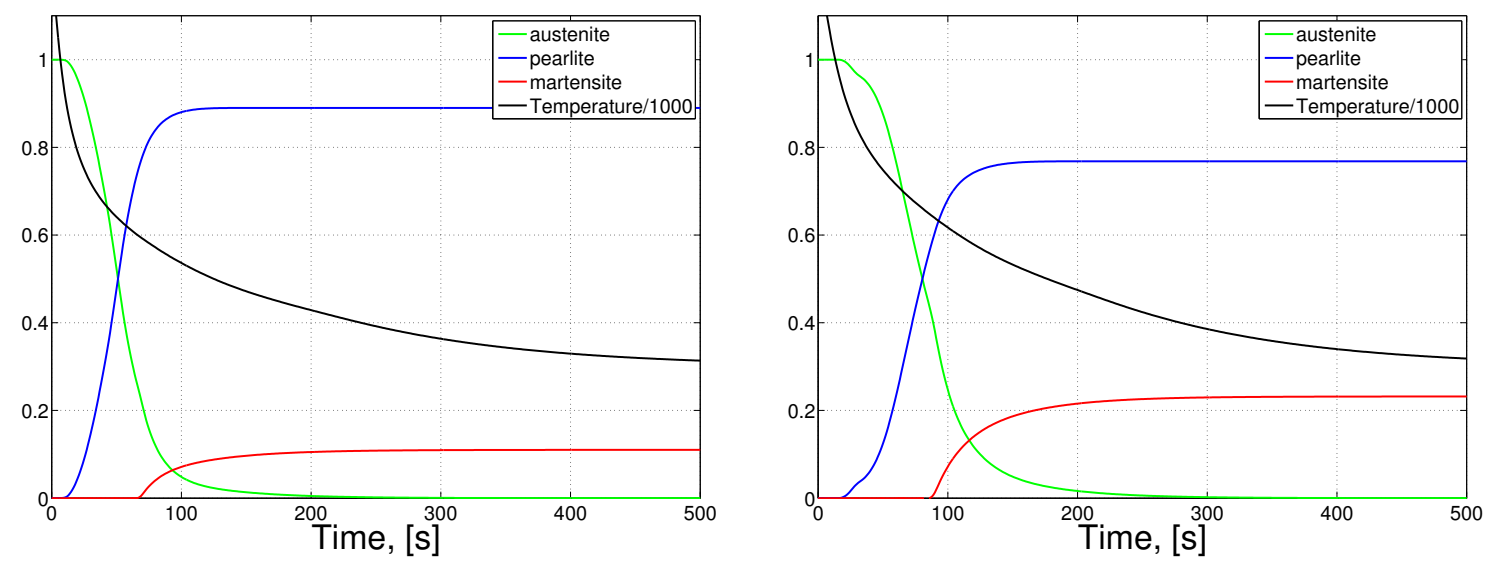

Phases and temperature evolution at point $d$. Phases and temperature evolution at point $e$.

Figure 4.12: Final phases distribution for an inhomogeneous carbon profile (upper left) and evolution of phases and temperature at five selected points.

From Figure 4.12 it appears that the final phases distribution is not monotonic with respect to the space, but presents a non monotonicity around the point denoted with $d$. Looking the pictures in sequence it appears that until the point $d$ the behaviour is similar to the one shown in the case of constant carbon content, i.e., the amount of martensite is high at the boundary, decreasing gradually ; correspondingly the higher amount of pearlite is formed far from the boundary, where the cooling rate is lower. This course is however interrupted passing from point $d$ to point $e$, where it turns in the opposite direction, as it can be seen from the reduction of the pearlite level. The phenomenon relies indeed on the differences in carbon content: corresponding to point $d$ is $0.4 \%$ and corresponding to $e$ is about $0.27 \%$.

Although a correct interpretation of the phase transformations during cooling would require the continuous cooling diagrams, we still can have some qualitative indications from the TTT diagrams in Figure 4.2. There, one can see that to a decrease in carbon percentage there corresponds a shift to the right of the bulge in the curve denoting the end of the pearlite formation. This results in the fact that more time is needed for the complete formation of pearlite, or, equivalently, in a reduced amount of pearlite producible in a certain interval of time.

In other words, from the diagrams we can infer that when the martensite formation starts, the percentage of pearlite formed at point $e$ is lower than the percentage at the point $d$. In our pictures we see that, at point $d$, the maximal percentage of pearlite of almost $90 \%$, whereas at point $e$ this maximum is around $0.75 \%$.

Other carbon configurations between the constant cases $0.2 \%$ and $0.5 \%$, could also be wished to suit particular uses; in Figure 4.13 we show three possible profiles and the corresponding final phase distributions. 


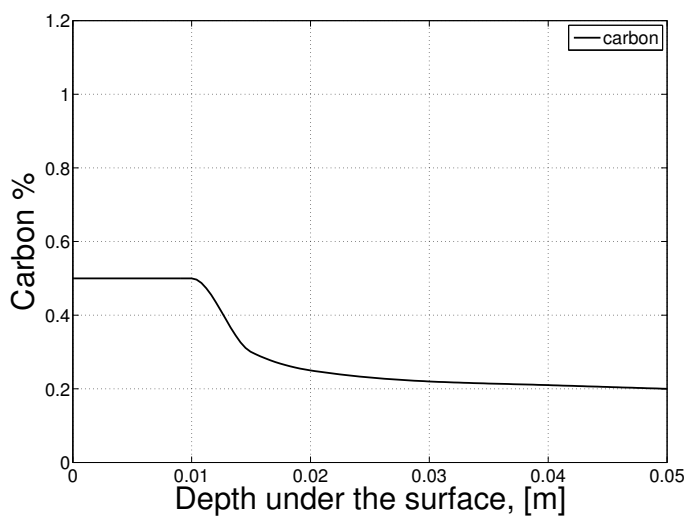

Carbon distribution $a$.

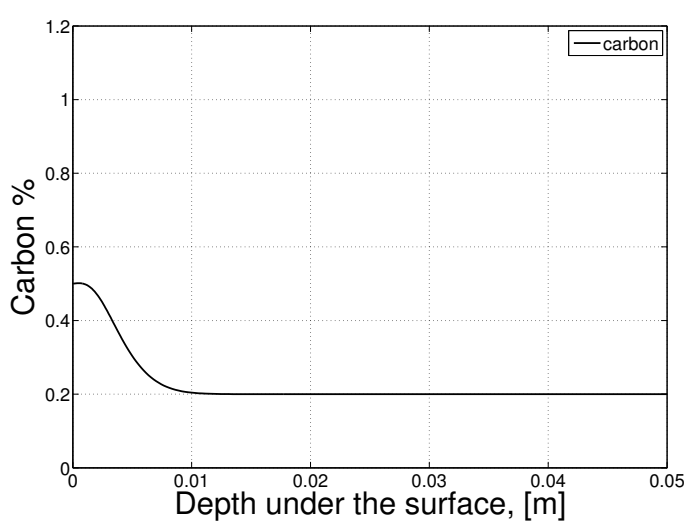

Carbon distribution $b$.

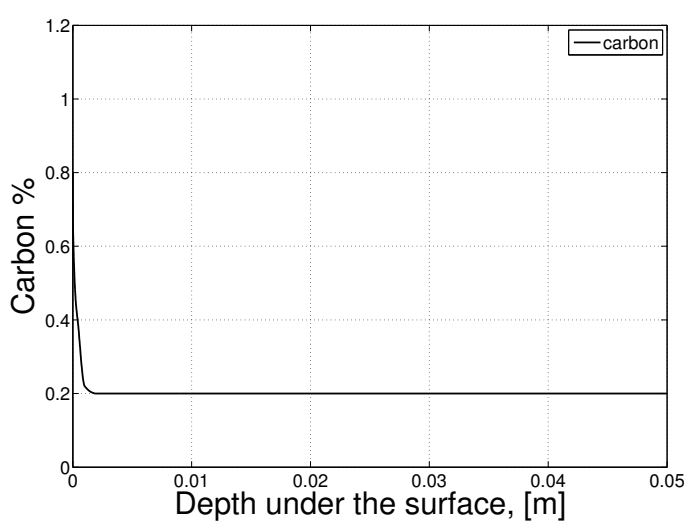

Carbon distribution $c$.

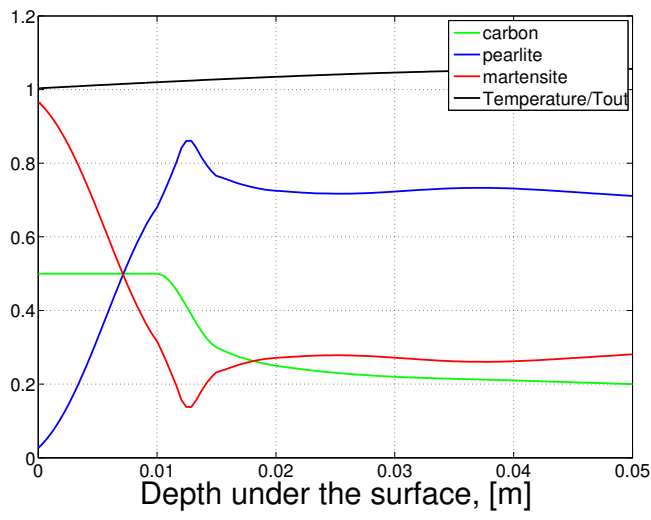

Final distribution, relative to carb. dist. $a$.

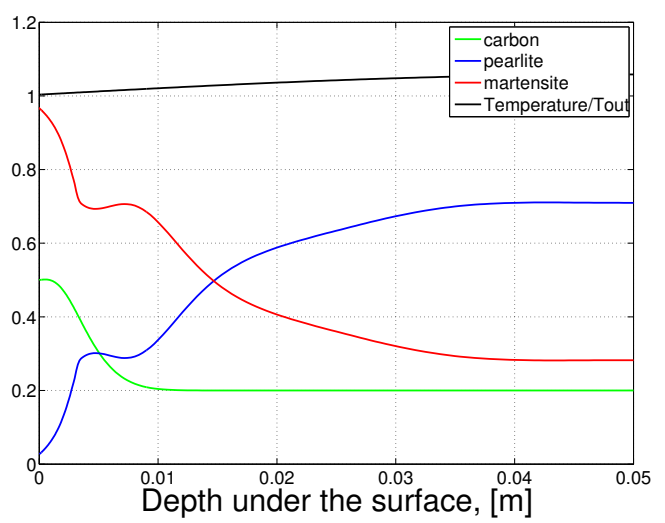

Final distribution, relative to carb. dist. $b$.

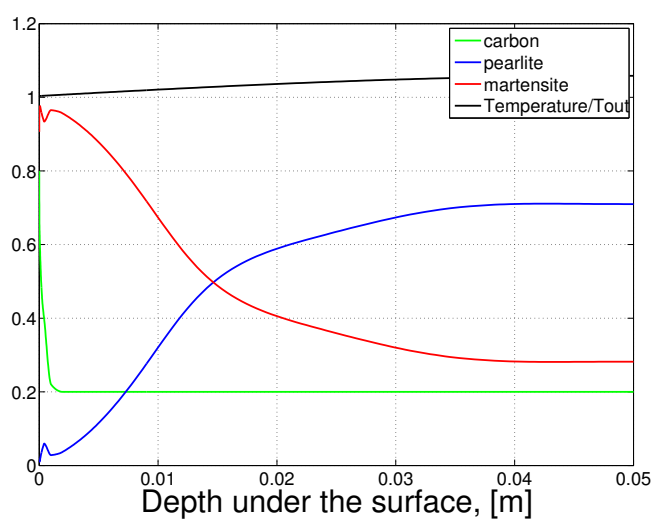

Final distribution, relative to carb. dist. $c$.

Figure 4.13: Three different carbon profiles with relatives final phase distributions.

\subsection{Simulation of the complete process}

In conclusion of the chapter, we consider a more complex geometry, namely a gear, which is a mechanical component often subject to case hardening. In this case we performed the simulations of the complete process. For reason of symmetry, it is 
enough to consider a section of the gear with half a tooth (see Figure 4.14 and 4.15).

We employ the same set of parameters as for the one dimensional simulations $\left(T_{0}=1228 \mathrm{~K}\right.$, $h=20000 \mathrm{~W} / \mathrm{m}^{2} \mathrm{~K}$ ) on the domain $\Omega$ is visible in Figure 4.15, of length $14 \mathrm{~cm}$ and we suppose that the carbon and the heat fluxes are active on the side of $\Omega$ representing the tooth, where the mesh is refined in order to capture what happens in the very thin external boundary layer. We assume $t_{1}=t_{2}=10000 \mathrm{~s}$ and $\mathrm{T}=10500 \mathrm{~s}$.

With these simulations we focus on the effect of the carbon potential, which is a determinant parameter during carburization and, together with the final martensite distribution, determines the final hardness at room temperature.

Four different carbon potentials $c_{p}$ relevant in the practice $(0.2 \%, 0.4 \%, 0.6 \%, 0.8 \%)$ were employed. Experimentally it can be seen that the maximal hardenability is achieved indeed for carbon content around $0.8 \%$ and that higher carbon amounts do not favour hardness, therefore values of $c_{p}$ higher than $0.8 \%$ are not meaningful. The next
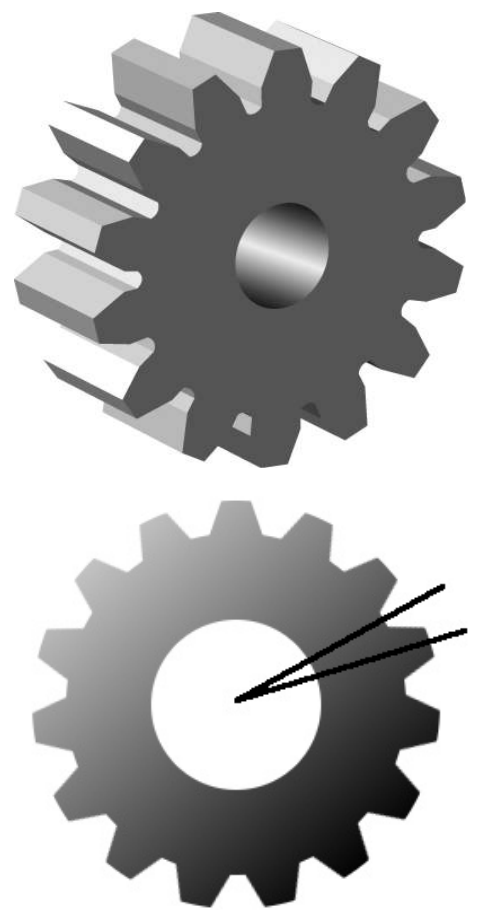

Figure 4.14: Steel gear (top) and its horizontal section (bottom). simulations show the effect of the different carbon potentials through its influence on the final martensite and carbon distribution. The relation between carbon content, martensite amount and hardness is in Figure 4.21. Figure 4.15 shows the mesh used and the final temperature distribution. Figure 4.16 is a view of the results of the simulations performed with $c_{p}=0.2 \%$

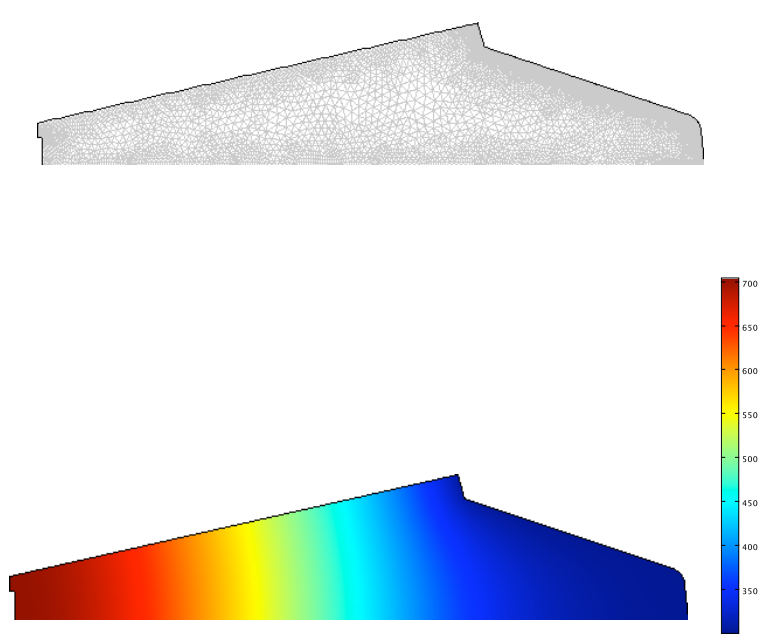

Figure 4.15: Used mesh (top), final temperature distribution (bottom). Colour indicates the temperature value in Kelvin. 

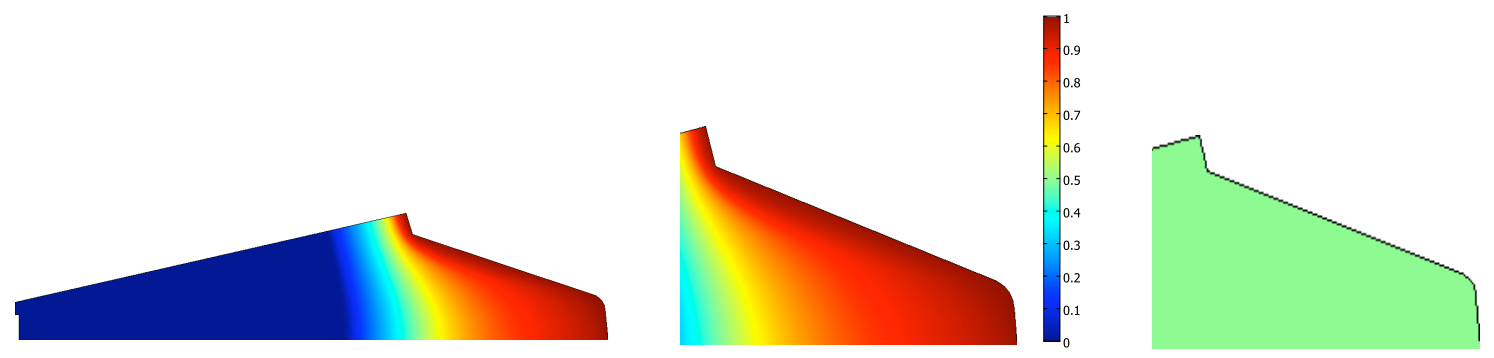

Figure 4.16: Results obtained with $c_{p}=0.2 \%$ : final martensite distribution (left), enlargement (centre), carbon distribution (right) where green colour means $0.2 \%$. The legend refers to the picture in the middle.

Figure 4.16, with the colour green, explains the absence of an effective carbon diffusion, since the steel has $0.2 \%$ carbon and $0.2 \%$ is also the carbon present in the atmosphere. On the other hand, in Figure 4.17, where the final martensite and carbon distributions are plotted in $0.4 \%, 0.6 \%, 0.8 \% c_{p}$ sequence, the carbon distribution, plotted in the right column, is inhomogeneous. The diffusion occurs only in a very thin boundary layer, around $0.5 \mathrm{~mm}$ thick and no difference is recognizable for different carbon potentials, whereas slight but visible changes in the marteniste distribution can be seen near the boundary.
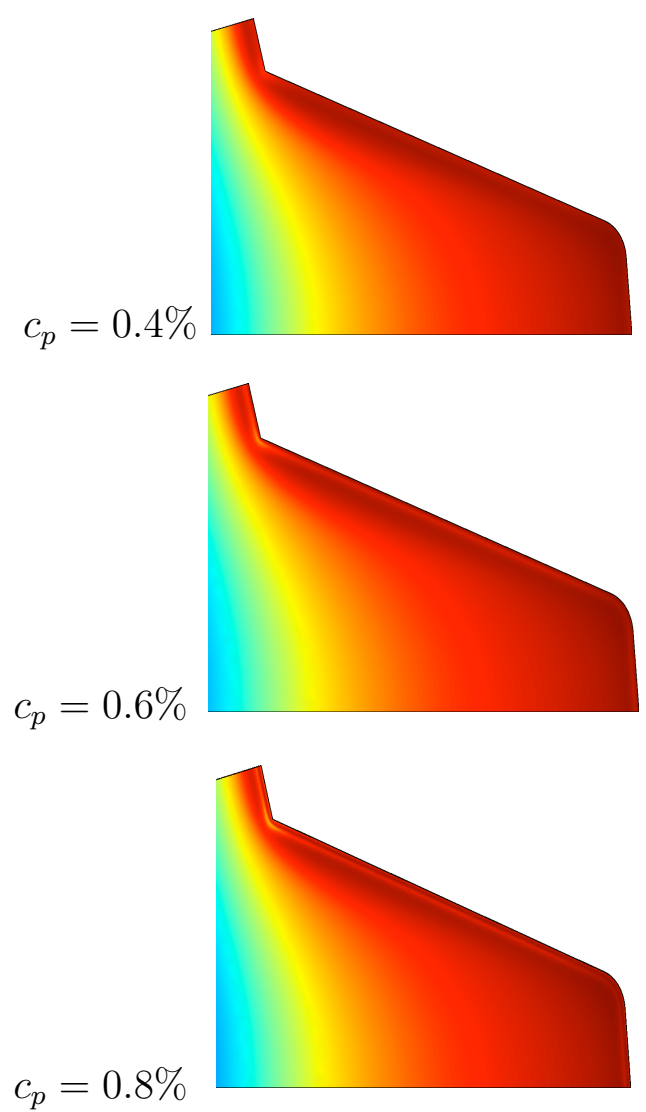
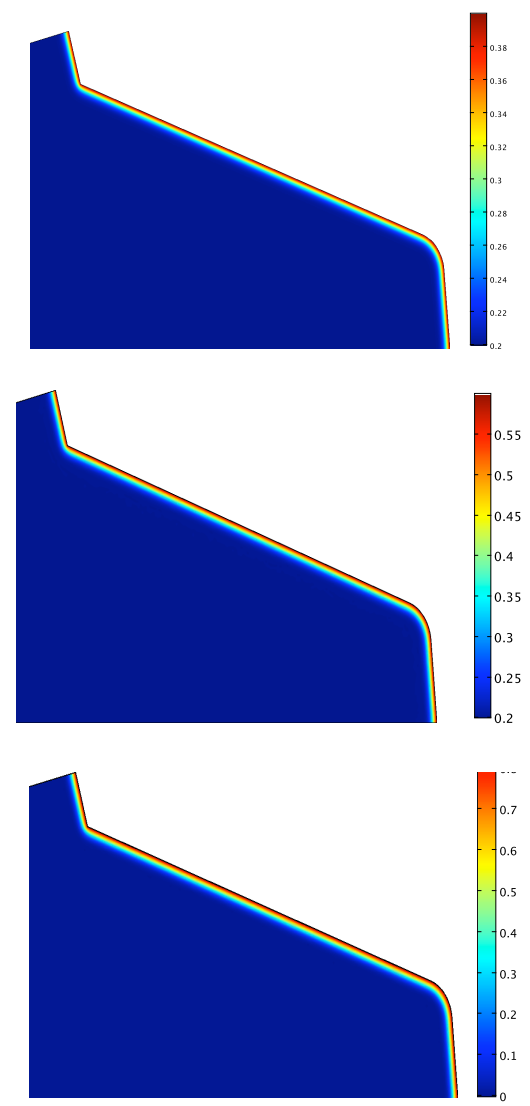

Figure 4.17: Pictures of the final martensite (left column) and carbon (right column) distribution in an enlargement around the carburized layer, starting from the top with carbon potential $c_{p}=0.2 \%$ to the bottom with $c_{p}=0.8 \%$. 
To enlighten the differences present in the pictures of Figure 4.17, we select now two segments (see Figure 4.18) along which we plot the martensite and carbon percentage, in Figure 4.19.

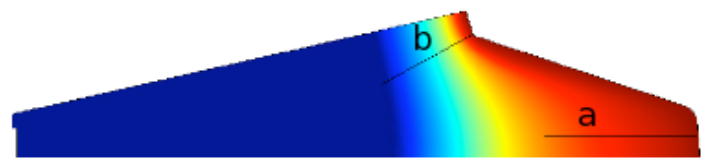

Figure 4.18: Segments $a$ and $b$ depicted on the total view of the final martensite distribution (obtained with $c_{p}=0.2$ ).

Profiles along the segment $a$

$c_{p}=0.2 \%$

$c_{p}=0.4 \%$

$c_{p}=0.6 \%$

$c_{p}=0.8 \%$
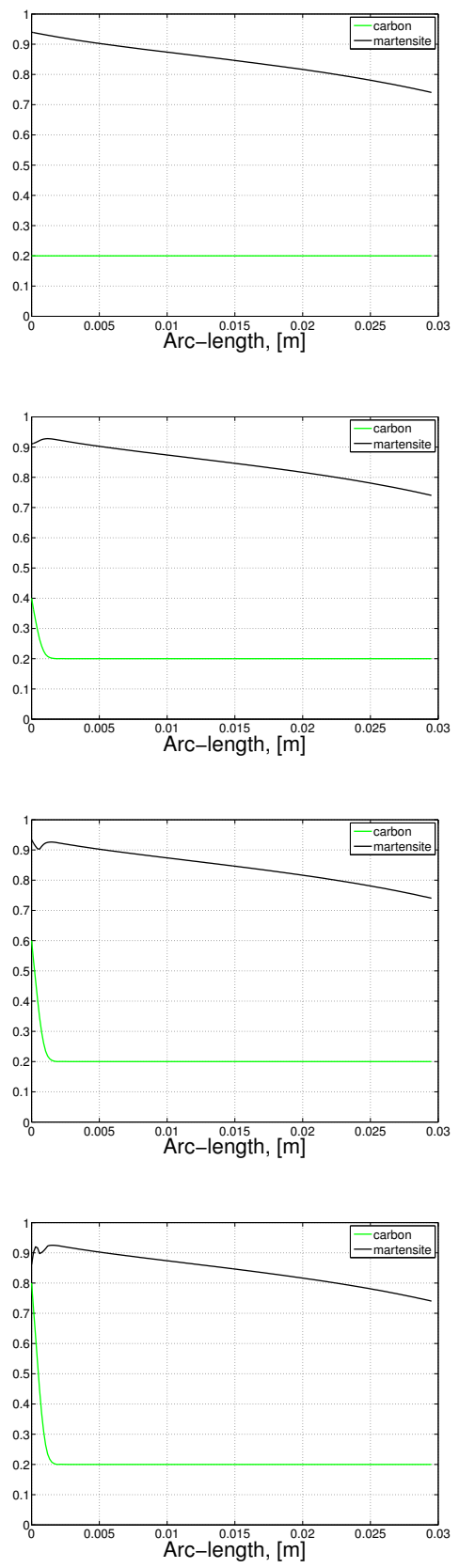

Profiles along the segment $b$
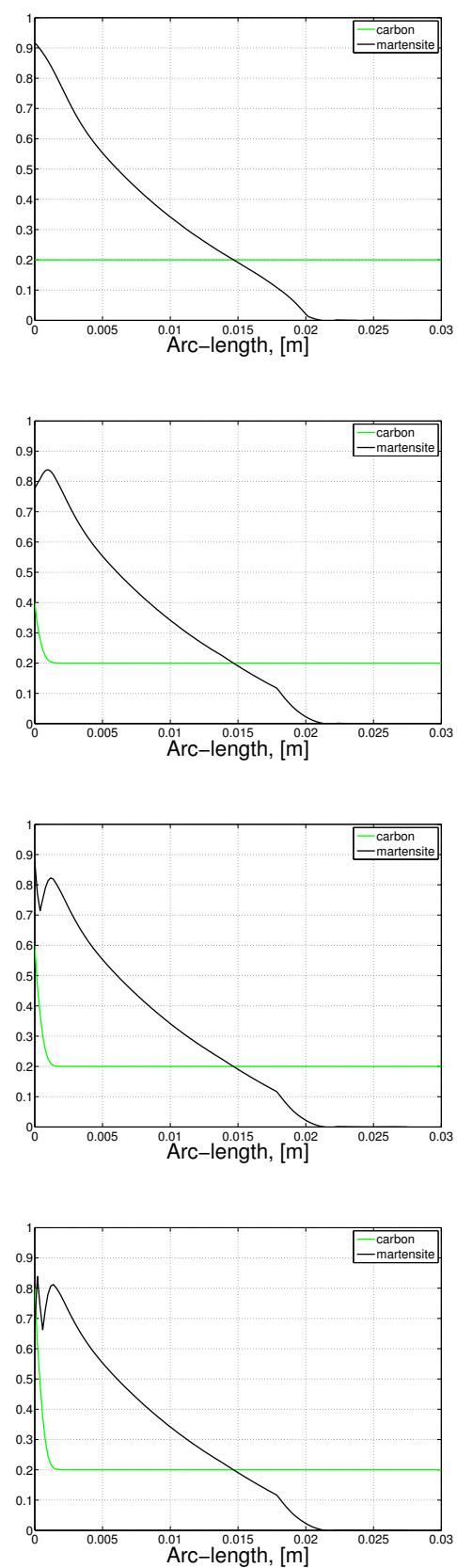
Figure 4.19: Plot of martensite and carbon percentages along the segments $a$ (left) and $b$ (right).

Both segments are $2 \mathrm{~cm}$ long. Along the segment $a$, the martensite fraction is $0.9 \%$ on the boundary and less lower at the depth of $2 \mathrm{~cm}$; the non monotonic profile is explicable in the same way as in the one dimensional case. Along the segment $b$ the martensite fraction decays more rapidly. This difference between the case $a$ and $b$ reflects the different curvatures in the workpiece.

We conclude with a short consideration about the final hardness. As it can be seen in Figure 4.20, it is possible to achieve different hardening depths, depending on the requests: the grey zone indicates the hardened part. The hardness measurements are obtained experimentally (cf. [5] e.g.). Figure 4.21 shows, schematically, the dependence of hardness on the martensite fraction and on the carbon content. It clearly appears that the maximum hardness is attained for $99 \%$ martensite and $0.8 \%$ carbon percent. For this reason a carbon potential higher than $0.8 \%$ is considered in most cases useless. In other words, a high amount of martensite does not provide high hardness if the carbon level is low. The resulting hardness depth in our two dimensional simulations, in view of the diagram in Figure 4.21, is qualitatively as the one in the right in Figure 4.20, where the entire gear tooth has been hardened.
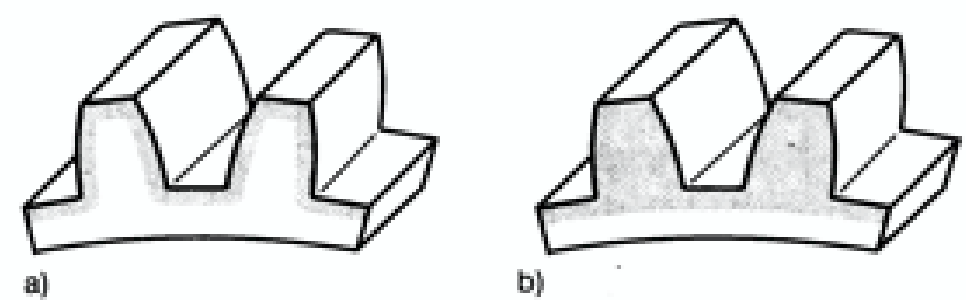

Figure 4.20: Two different hardening depths.

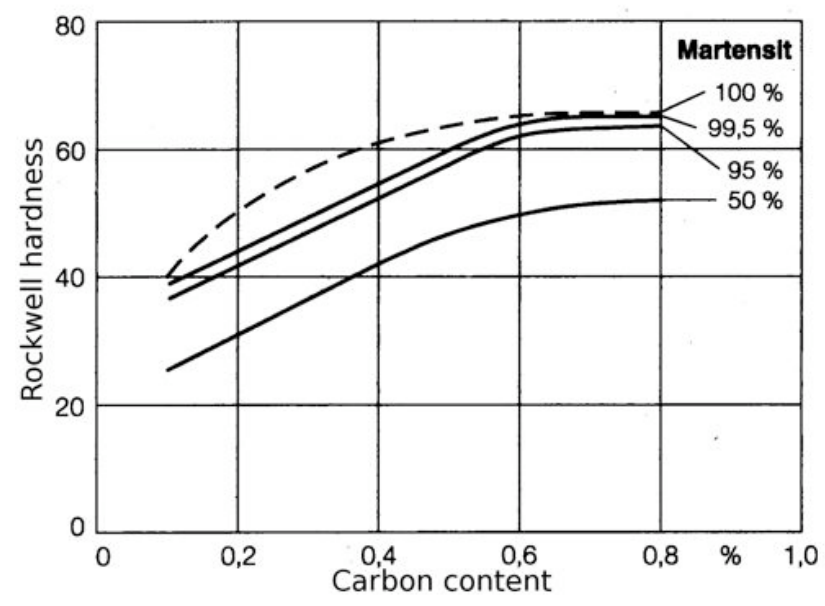

Figure 4.21: Relation between the carbon content (x-axis) and the hardenability (y-axis), depending on the martensite amount after hardening (from [5]). 
In conclusion, the purpose of the simulation work of this chapter was to show a possible concrete application of the proposed model. In absence of direct experimental confirmations, we based our simulation on realistic data taken from literature and we have performed numerical simulations using a software based on finite-element method first for a one-dimensional geometry and then for a sector of a gear.

The simulations confirmed, on one side, the expected scenarios in the case of constant carbon content and, on the other side, show interesting phenomena due to the presence of a inhomogeneous carbon distribution, that we have investigated with the help of the time-temperature-transformation diagrams for the considered steel. Under this regard, a cooperation with some engineering institute could be very desirable in order to compare our simulations with the experiments. 


\section{Chapter 5}

\section{Conclusions}

We have modelled carbon diffusion, heat conduction and phase transformations in steel and developed a macroscopic mathematical model for a special variant of case hardening, called gas carburizing. In our model steel was considered as a co-existing mixture of phases, which do not diffuse but rather stay in the position where they have grown. The model allow to describe, from a phenomenological point of view, the kinetics of carburization and the subsequent transformation during cooling. It consists of a coupled system of two parabolic equations for carbon diffusion through the austenite and for heat conduction and ordinary differential equations for the evolution of the phase fractions.

The complete model has been analysed, covering all the stages of the process, and it was shown, under suitable assumptions, the existence of a weak solution through a nested fixed point argument. Under stronger assumptions, also the uniqueness of a solution has been proven.

Next we examined a related problem constituted by a quasilinear system of two parabolic equations with nonlinear boundary conditions, since it was reckoned of intrinsic mathematical interest. Also in this case, we examined the question of the existence of a weak solution of the corresponding initial-boundary value problem and we focused in particular on the question of the uniqueness of the solutions, trying to allow weaker assumptions than those necessary for the whole system. In order to do that, the regularity of the solutions has been investigated. A major achievement is the technique developed to prove the necessary regularity in order to accomplish the proof of uniqueness. We concluded the chapter with an embedding theorem for a wide class of anisotropic Sobolev spaces, that we believe to be of its own interest, which contains as special cases some embedding theorems in the classical Sobolev spaces.

Lastly we have demonstrated how to apply the model developed to a concrete situation: for a one dimensional configuration we have performed numerical simulations. Despite its simplicity, this configuration provides a very useful insight in the comprehension of the behaviour of the phases and of carbon. We selected a specific type of steel for which data were available from literature. Still we had to face the question of how to determine the phase transition rates as functions of the carbon concentration. We bypassed this obstacle using appropriately the available time-temperature- 
transformation diagrams for the steel considered. The peculiarities due to the presence of an in-homogeneous carbon content have been pointed out and investigated. Finally we showed the simulations performed taking as sample work-piece a gear sector and showed that a thorough description of the process of carburization was obtained.

\section{Future research}

There are natural developments for future research both in mathematical sense and in the direction of the applications. From the point of view of the possible application of our model, a co-operation with engineering research institutes would be of great advantage, to enlighten and detail the numerical simulation aspect. The comparisons with the experimental results would require indeed more precise data. The difficulty encountered in this sense, during the development of the thesis, is due to the fact that an exhaustive databank for the material behaviour of steel is still the aim of very active research in the engineering field nowadays and it is not a simple question.

From the point of view of modelling and mathematics, there are other relevant questions that could be investigated. In our study, mechanical deformations are neglected and the distortion, in particular the one related to case hardening, can not be described by the proposed model. The modelling of material behaviour of steel is a large field of current research. Because of the high complexity of some material phenomena of steel, as for example deformations, plasticity, transformation induced plasticity, phase transformation, e.g., it is common practice to study these phenomena by experiments in more or less isolated situations in order to find the basic relations and then to include them in a bulk model of material behaviour, as the one we presented.

In fact, the model treated in the present thesis can be extended via a building-block principle, extending it possibly to a more general material behaviour of steel, including also the mechanical effects. In order to capture the distortion effect, it is necessary to add a momentum balance as well as additional constitutive equations to the model. For modelling of mechanical behaviour of steel including carbon diffusion and phase transformations, we refer to [26], [27] and references therein.

Another interesting research subject, equally important for the mathematical aspects and the applications, is how to optimize the carburizing process. Indeed, controlling gas carburizing still presents many difficulties, since all the different phenomena occurring on the steel surface have a crucial influence and it is quite complicated trying to drive the whole process to a desired target. Nonetheless, optimization techniques are strongly requested in order to meet quality requirements, by shortening cycle time, enhancing furnace capacity and thereby reducing the energy consumption. In many installations, certain parameters such as the atmosphere composition and the time of exposure are fixed, while the external temperature and the carbon potential are varied to achieve different targets. An ideal objective would be to formulate an optimal control strategy aimed at obtaining a desired carbon profile and a desired temperature at the end of the process. A first approach would consists therefore in minimizing a suitable cost functional subjected to the state equations (1.4.6)-(1.4.15), with control 
parameters the carbon potential, previously denoted with $c_{p}$ and the external temperature, $\theta_{\Gamma}$. We can expect that, due to the quasilinearity of system (1.4.6)-(1.4.15), in conjuction with the further requests coming form the specificity of the optimal control problem treatment, the analysis would pose many difficulties. Globally this can be foreseen to result in challenging mathematical problem with an immediate relevant application. 


\section{Bibliography}

[1] H. Amann. Linear and quasilinear parabolic problems. Birkhauser, 1995.

[2] D. Andreucci, A. Fasano, M. Primicerio. On a mathematical model for the crystalization of polymers. in Proceed. 4th Europ. Conf. Math. in Industry (Hj. Wacker, W. Zulehner eds.), Teubner, Stuttgart, (1991), 3-16.

[3] S.H. Avner. Introduction to physical metallurgy. Second Edition, McGraw-Hill Book Company, 1974.

[4] M. Avrami. Kinetics of phase change. Chem. Phys., (1940), 7-9.

[5] H. J. Bargel, G. Schulze Werkstoffkunde. Springer, 2005.

[6] O. V. Besov, V. P. Il'in, S. M. Nikol'skiǔ. Integral Representations of Functions and Imbedding Theorems. Scripta Series in Mathematics, Halsted Press (John Wiley \& Sons), New York-Toronto, Ont.-London, 1978 (Vol. I), 1979 (Vol. II). Russian version Nauka, Moscow, 1975.

[7] H. E. Boyer. Practical Heat Treating. American Society for Metals, 1984.

[8] H. Brezis. Analyse fonctionelle: Théorie et applications. Masson, 1983.

[9] R. Chatterjee-Fischer. Überblick über die Möglichkeiten zur Verkürzung der Aufkohlungsdauer. HTM Härterei-Techn. Mitt., 40, 1 (1985), 7-11.

[10] J. W. Christian. The theory of transformations in metals and alloys. Part I. Pergamon Press, Oxford, 1985.

[11] A. Coddington, N. Levinson. Theory of ordinary differential equations. New York, McGraw-Hill, 1955.

[12] R. Collin, S. Gunnarson, D. Thulin. A mathematical model for predicting carbon concentration profiles of gas-carburized steel. J. Iron Steel Inst., (1972), 785-789.

[13] S. Denis, D. Farias, A. Simon. Mathematical model coupling phase transformations and temperature evolutions in steel. ISIJ International, 32 (1992), 316-325.

[14] L. C. Evans, Partial differential equations. Graduate Studies in Mathematics, V. 19, American Mathematical Society, 1998.

[15] J.A. Griepentrog. Sobolev-Morrey spaces associated with evolution equations. Adv. in Diff. Eq., 12 (2007), 781-840; Maximal regularity for nonsmooth parabolic problems in Sobolev-Morrey spaces. Adv. in Diff. Eq., 12 (2007), 1031-1078. 
[16] E. L. Gyulikhandanov, V. V. Kislenkov, S. P. Provotorov. Computing the concentration profile of carbon during the carburizing of steels in controlled natural-gas atmosphere. Translated from Metallovedenie i Termicheskaya Orabotka Metallov, 8 (1981), 9-11.

[17] F. E. Harris. Case depth - an attempt at a practical definition. Met. Prog., 44 (1943), $265-272$.

[18] M. Hieber, J. Rehberg. Nonlinear parabolic systems with mixed boundary conditions. SIAM J. Math. Anal., 40 (2008), 292-305.

[19] D. Hömberg. A mathematical model for the phase transitions in euctectoid carbon steel. IMA J. Appl. Math., 54 (1995), 31-57.

[20] D. Hömberg, A mathematical model for induction hardening including mechanical effects. Nonlinear Anal. Real World Appl., 5 (2004), 55-90.

[21] D. Hömberg. Irreversible phase transitions in steel. Math. Methods Appl. Sci., 20 (1997), 59-77.

[22] D. Hömberg, A. Fasano, L. Panizzi. A mathematical model for case hardening of steel. Math. Models Meth. Appl. Sci., (2009), in press.

[23] D. Hömberg, W. Wolff. PID-control of laser surface hardening of steel. IEEE Trans. Control Syst. Technol., 14 (2006), 896-904.

[24] H.P. Hougardy. Die Darstellung des Umwandlungsverhaltens von Stählen in den ZTUSchaubildern. HTM Härterei-Techn. Mitt., 33, 2 (1978), 63-70.

[25] M. Hunkel, T. Lübben, F. Hoffmann, P. Mayr. Using the jominy end-quench test for validation of thermo-metallurgical model parameters. J. Phys. IV France, 120 (2004), $571-579$.

[26] T. Inoue, T. Yamaguchi, Z. Wang. Stresses and phase transformations occurring in quenching of carburized steel gear wheel. Mat. Sci. and Tech., 1 (1985), 872-876.

[27] T. Inoue, Z. Wang, K. Miyao. Quenching stress of carburized steel gear wheel, in Proceed. ICRS2, (G. Beck, S. Denis, A. Simon eds), Elsevier Appl. Sci. London, New York, (1989), 606-611.

[28] H.W. Knobloch, F. Kappel. Gewöhnliche Differentialgleichungen. Teubner, 1974.

[29] D. P. Koistinen, R. E. Marburger. A general equation prescribing the extent of the austenite-martensite transformation in pure iron-carbon alloys and plain carbon steel. Acta Met., 7 (1959), 59-60.

[30] A. Kolmogorov. Statistical theory of crystallization of metals. Bull. Acad. Sci. USSR Mat. Sci., 1(1937), 355-359.

[31] A. Koshelev. Regularity Problem for Quasilinear Elliptic and Parabolic Systems. Lecture Notes in Mathematics, Springer-Verlag, Berlin, 1995.

[32] P. Krejčí, L. Panizzi. Regularity and uniqueness in quasilinear parabolic systems. Applications of mathematics, submitted. 
[33] O.A. Lady̆zenskaja, V.A. Solonnikov and N.N. Ural'ceva. Linear and Quasilinear Equations of Parabolic Type. Amer. Math. Soc. Transl. 23, AMS, Providence, RI, 1968.

[34] J. B. Leblond, J. Devaux. A new kinetic model for anisothermal metallurgical transformations in steel including effect of austenite grain size. Acta Met., 32 (1984), 137-146.

[35] G.M. Lieberman. Second order parabolic differential equations. World Scientific Publishing Company, 1996.

[36] J.L. Lions. Quelques méthodes de résolution des problèmes aux limites non linéaires. Dunod; Gauthier-Villars, Paris 1969.

[37] J.L. Lions, E. Magenes. Problémes aux limites non homogénes, Vol II. Dunod, 1968.

[38] J. Lütjens, V. Heuer, F. König, T. Lübben, V. Schulze, N. Trapp. Computer Aided Simulation of Heat Treatment (C.A.S.H.) Teil 2: Bestimmung von Eingabedaten zur FEMSimulation des Einsatzhärtens. HTM Z. Werkst. Waermebeh. Fertigung, 61, 1 (2006) $10-17$.

[39] M. Motoyama, R.E. Ricklefs, J.A. Larson. The effect of carburizing variables on residual stresses in hardened chromium steel, Automotive Engineering Congress and Exposition Detroit, Michigan, Feb. 24-28. Paper number 750050, (1975).

[40] J. Nečas. Les méthodes directes en théorie des équations elliptiques. Academia, Prague, 1967.

[41] A. Ochsner, J. Gegner, G. Mishuris. Effect of diffusivity as a function of the method of computation of carbon concentration profiles in steel. Met. Sci. Heat. Treat., 46 (2004), $3-4$.

[42] J. F. Rodrigues. A nonlinear parabolic system arising in thermomechanics and in thermomagnetism. Math. Models Methods Appl. Sci., 2 (1992), 271-281.

[43] T. Shilkin. Classical solvability of the coupled system a heat-convergent Poiseuille-type flow. J. Math. Fluid Mech. 7 (2005), 72-84.

[44] C.A. Stickels. Gas carburizing, Heat Treatment, Vol. 7, American Society for Metals, (1997), 312-324.

[45] C.A. Stickels. Analytical models for the gas carburizing process, Metall. Trans. B, 20B (1989).

[46] T. Turpin, J. Dulcy, M. Gantois. Carbon diffusion and phase transformations during gas carburizing of high-alloyed stainless steels: experimental study and theoretical modeling, Metall. and Mat. Trans. A, 36A (2005), 2751-2759.

[47] G.F. Vander Voort. Atlas of time-temperature diagrams for iron and steels, ASM International, 1991.

[48] Verein Deutscher Eisenhütteleute. Steel, a Handbook for Material Research and Engineering, Vol. 1-2, Springer, 1993.

[49] A. Visintin. Mathematical models of solid-solid phase transitions in steel. IMA J. Appl. Math., 30 (1987), 143-157. 
[50] M. Wolff, C. Acht, M. Böhm, S. Meier. Modelling of carbon diffusion and ferritic phase transformations in an unalloyed hypoeutectoid steel. Arch. Mech., 59 (2007), 1-33.

[51] http:// www.lesman.com Application Note: Carbon Potential Control. Lesman Company.

[52] E. Zeidler, Nonlinear Functional Analysis and Its Applications Vol II. Springer-Verlag, 1990. 